\title{
REFLECTION GROUPS IN ALGEBRAIC GEOMETRY
}

\author{
IGOR V. DOLGACHEV
}

To Ernest Borisovich Vinberg

\begin{abstract}
After a brief exposition of the theory of discrete reflection groups in spherical, euclidean and hyperbolic geometry as well as their analogs in complex spaces, we present a survey of appearances of these groups in various areas of algebraic geometry.
\end{abstract}

\section{INTRODUCTION}

The notion of a reflection in a euclidean space is one of the fundamental notions of symmetry of geometric figures and does not need an introduction. The theory of discrete groups of motions generated by reflections originates in the study of plane regular polygons and space polyhedra, which goes back to ancient mathematics. Nowadays it is hard to find a mathematician who has not encountered reflection groups in his area of research. Thus a geometer sees them as examples of discrete groups of isometries of Riemannian spaces of constant curvature or examples of special convex polytopes. An algebraist finds them in group theory, especially in the theory of Coxeter groups, invariant theory and representation theory. A combinatorialist may see them in the theory of arrangements of hyperplanes and combinatorics of permutation groups. A number theorist meets them in arithmetic theory of quadratic forms and modular forms. For a topologist they turn up in the study of hyperbolic real and complex manifolds, low-dimensional topology and singularity theory. An analyst sees them in the theory of hypergeometric functions and automorphic forms, complex higher-dimensional dynamics and ordinary differential equations. All of the above and much more appears in algebraic geometry. The goal of this survey is to explain some of "much more".

One finds an extensive account of the history of the theory of reflection groups in euclidean and spherical spaces in Bourbaki's Groupes et Algèbres de Lie, Chapters IV-VI. According to this account the modern theory originates from the works of geometers A. Möbius and L. Schläfli in the middle of the 19th century, then was extended and applied to the theory of Lie algebras in the works of E. Cartan and W. Killing at the end of the same century, and culminated in the works of H. S. M. Coxeter [27]. The first examples of reflection groups in hyperbolic plane go back to F. Klein and H. Poincaré at the end of the 19th century.

Received by the editors December 3, 2006, and, in revised form, May 17, 2007.

2000 Mathematics Subject Classification. Primary 20F55, 51F15, 14E02; Secondary 14J28, 14E07, 14H20, 11H55.

The author was supported in part by NSF grant no. 0245203. 
It is not general knowledge that reflection groups, finite and infinite, appeared in 1885-1895 in the works of S. Kantor [63] on classification of subgroups of the Cremona group of birational transformations of the complex projective plane [63]. He realized their reflection action in the cohomology space of rational algebraic surfaces obtained as blow-ups of the plane. In this way all Weyl groups of type $A_{2} \times A_{1}, A_{4}, D_{5}, E_{6}, E_{7}, E_{8}$ appear naturally when the number of points blown up is between 3 and 8 . The last three groups appeared much earlier in algebraic geometry as the group of 27 lines on a cubic surface (type $E_{6}$ ), the group of bitangents of a plane quartic (type $E_{7}$ ), and the group of tritangent planes of a space sextic of genus 4 (type $E_{8}$ ). They were widely known among algebraists since the appearance of C. Jordan's "Traité des substitutions" in 1870. In 1910 P. Schoute discovered a convex polytope in six-dimensional space whose vertices are in a bijective correspondence with 27 lines on a cubic surface and the group of symmetries is isomorphic to the group of 27 lines [98]. A similar polytope in seven-dimensional space was found for the group of 28 bitangents of a plane quartic by Coxeter [26]. The relationship between this six-dimensional space and the cohomology space of the blow-up of the plane at 6 points was explained by P. Du Val in the 1930s. He also showed that all Kantor groups are reflection groups in euclidean, affine or hyperbolic spaces [39], [38].

The Coxeter diagram of the Weyl group of type $E_{8}$ is of the form

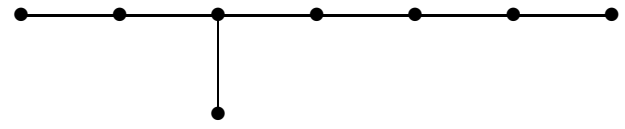

Figure 1

It appears when the number of points blown up is equal to 8 . When the number of points is 9 , we get a reflection group in affine space of dimension 8 , the affine Weyl group of type $E_{8}$. Its Coxeter diagram is obtained by adding one point in the long arm of the diagram above. Starting from 10 points one gets Coxeter groups in hyperbolic space with Coxeter diagram of type $E_{n}$ (extending the long arm of the diagram in Fig. 1). In 1917, generalizing Kantor's work, Arthur Coble introduced the notion of a regular Cremona transformation of a higher-dimensional projective space and considered more general Coxeter groups of type $W(2, p, q)$ [21]. Its Coxeter diagram is obtained from extending the two upper arms of the diagram. A modern account of Coble's theory is given in my book with D. Ortland [36]. Very recently, S. Mukai [82] was able to extend Coble's construction to include all Coxeter groups of type $W(p, q, r)$ with Coxeter diagram obtained from the above diagram by extending all its arms.

Another class of algebraic surfaces where reflection groups in hyperbolic spaces arise naturally is the class of surfaces of type K3. An example of such a surface is a nonsingular quartic surface in complex three-dimensional projective space. F. Severi gave the first example of a quartic surface with an explicitly computed infinite group of birational automorphisms; the group turns out to be isomorphic to a plane reflection group [100]. In 1972 I.I. Pyatetsky-Shapiro and I. R. Shafarevich, answering a question of $\mathrm{A}$. Weil, proved that the complex structure of an algebraic (polarized) K3-surface is determined uniquely by the linear functional on its second cohomology space obtained by integrating a nowhere vanishing holomorphic 2 -form 
[94], [101]. They called this result a Global Torelli Theorem. As a corollary of this result they showed that the group of automorphisms of a K3 surface is isomorphic, up to a finite group, to the quotient of the orthogonal group of the integral quadratic form defined on the group of integral algebraic 2-cycles modulo the subgroup $\Gamma$ generated by reflections in the cohomology classes of smooth rational curves lying on the surface. Thus they reduced the question of finiteness of the automorphism group to the question of finiteness of the volume of the fundamental polyhedron of $\Gamma$ in a real hyperbolic space. V. Nikulin [87] and E. Vinberg [112] determined which isomorphism types of integral quadratic forms so arise and have the property that the fundamental polyhedron in question has finite volume. This solves, in principle, the problem of classification of fields of algebraic dimension 2 over $\mathbb{C}$ whose group of automorphisms over $\mathbb{C}$ is infinite.

In the 1930s Patrick Du Val [37] found the appearance of Coxeter diagrams in resolution of certain types of singularities on algebraic surfaces (nowadays going under the different names: simple singularities, ADE singularities, Du Val singularities, double rational points, Gorenstein quotient singularities, and Klein singularities). However, Du Val did not find any reflection groups associated to these singularities. A conjectural relation to reflection groups and simple Lie algebras was suggested by A. Grothendieck in the sixties and was confirmed by a construction of E. Brieskorn [16] (full details appeared in [105]).

The theory of finite complex reflection groups was developed by G. C. Shephard and A. Todd in 1954 as a follow-up to the classical work on groups of projective transformations generated by homologies (see [102]). Some examples of the arrangements of reflection hyperplanes and the hypersurfaces defined by polynomial invariants of the groups have been known in classical geometry since the 19th century.

Infinite reflection groups of finite covolume in complex affine spaces were classified by V. Popov in 1982 [93]. They appear in the theory of compactification of versal deformation of simple elliptic singularities [74] and surface singularities with symmetries [50].

The most spectacular is the appearance of reflection groups in complex hyperbolic spaces of dimension $>1$. Extending the work of H. Terada [109], P. Deligne and G. Mostow [28], [80] classified all hypergeometric functions whose monodromy groups $\Gamma$ are discrete reflection groups of finite covolume in a complex ball $\mathbb{B}^{r}$ (complex hyperbolic crystallographic groups, c.h.c. groups for short). The compactified orbit spaces $\overline{\mathbb{B}^{r} / \Gamma}$ turned out to be isomorphic to some geometric invariant quotients $\mathbb{P}^{1}(\mathbb{C})^{r+3} / / \mathrm{PGL}(2, \mathbb{C})$ for $r \leq 9$. No other c.h.c. groups in $\mathbb{B}^{r}$ had been discovered until a few years ago (except one missed case in Deligne-Mostow's list noticed by W. Thurston [110]). The first new c.h.c. group in $\mathbb{B}^{4}$ appeared in a beautiful construction of D. Allcock, J. Carlson and D. Toledo of a complex ball uniformization of the moduli space of cubic surfaces [2]. Later, using a similar uniformization construction for moduli spaces of other Del Pezzo surfaces, new examples of c.h.c, groups were found in dimensions 6 and 8 [56], [72], [71]. All these groups are commensurable with some of the Deligne-Mostow groups. Recent work of Allcock, Carlson and Toledo [4] (see also [77]) on complex ball uniformization of the moduli space of cubic hypersurfaces in $\mathbb{P}^{4}$ produces a new complex reflection group in dimension 10. A generalization of the Deligne-Mostow theory due to W. Couwenberg, G. Heckaman and E. Looijenga [25] gives other new examples of 
complex reflection crystallographic groups. A c.h.c. group in a record high dimension 13 was constructed by D. Allcock [1]. No geometrical interpretation so far is known for the corresponding ball quotients.

The above discussion outlines the contents of the present paper. As is the case with any survey paper, it is incomplete, and the omitted material is either due to the author's ignorance, poor memory, or size limitations of the paper.

\section{REAL REFLECTION GROUPS}

2.1. Elementary introduction. The idea of a reflection transformation $r_{H}$ with respect to a mirror line $H$ is of course very familiar. A picture on the plane is

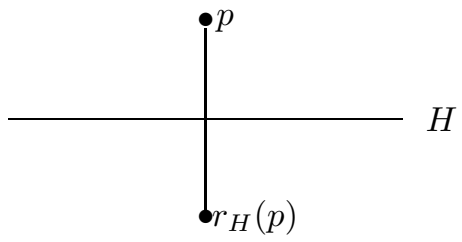

FiguRE 2

Now suppose we have two mirror lines $H_{1}$ and $H_{2}$. Each line divides the plane into the disjoint union of two halfplanes, $H_{i}^{ \pm}$.

A choice of halfplanes, say $H_{1}^{+}, H_{2}^{+}$, defines the angle $H_{1}^{+} \cap H_{2}^{+}$with measure $\phi=\angle\left(H_{1}^{+}, H_{2}^{+}\right)$. Here $\phi=0$ if and only if $H_{1}^{-} \cap H_{2}^{-}=\emptyset$.

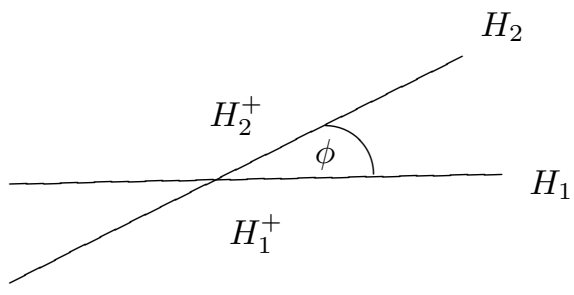

FigURE 3

Let $s_{1}=r_{H_{1}}, s_{2}=r_{H_{2}}$. The composition $s_{2} s_{1}$ is the counterclockwise rotation about the angle $2 \phi$ if $\phi \neq 0$ and a translation if $\phi=0$ :
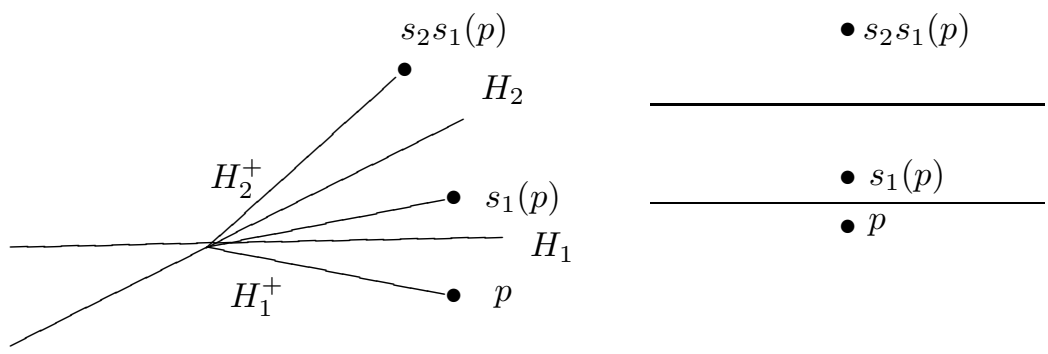
Let $G$ be the group generated by the two reflections $s_{1}, s_{2}$. We assume that $H_{1} \neq H_{2}$, i.e. $s_{1} \neq s_{2}$. The following two cases may occur:

Case 1: The angle $\phi$ is of the form $n \pi / m$ for some rational number $r=n / m$. In the following we assume that $m=\infty$ if $\phi=0$.

In this case $s_{2} s_{1}$ is the rotation about the angle $2 n \pi / m$ and hence

$$
\left(s_{2} s_{1}\right)^{m}=\text { identity. }
$$

The group $G$ is isomorphic to the finite dihedral group $D_{2 m}$ of order $2 m$ (resp. infinite dihedral group $D_{\infty}$ if $m=\infty$ ) with presentation

$$
<s_{1}, s_{2} \mid s_{1}^{2}=s_{2}^{2}=\left(s_{1} s_{2}\right)^{m}=1>.
$$

It acts as a discrete group of motions of the plane with fundamental domain $H_{1}^{-} \cap H_{2}^{-}$.

Observe that the same group is generated by reflections with respect to the lines forming the angle obtained from the angle $\angle\left(H_{1}^{-}, H_{2}^{-}\right)$, subdividing it into $n$ equal parts. So, we may assume that $\phi=\pi / m$.

Case 2: The angle $\phi$ is not of the form $r \pi$ for any rational $r$.

In this case $s_{2} s_{1}$ is of infinite order, $G$ is isomorphic to $D_{\infty}$, but it does not act discretely.

Now suppose we have a convex polygon given as the intersection of a finite set of halfplanes

$$
P=\bigcap_{i=1}^{r} H_{i}^{-} .
$$

We assume that the interior $P^{o}$ is not empty and the set $\left\{H_{1}, \ldots, H_{r}\right\}$ is minimal in the sense that one cannot delete any of the halfplanes without changing $P$.

More importantly, we assume that

$$
\angle\left(H_{i}^{-}, H_{j}^{-}\right)=\pi / m_{i j}
$$

for some positive integer $m_{i j}$ or equal to $0\left(m_{i j}=\infty\right)$.

Let $G$ be the group generated by reflections with mirror lines $H_{i}$. It is a discrete group of motions of the plane. The polygon $P$ is a fundamental domain of $G$ in the plane.

Conversely any discrete group of motions of the plane generated by reflections is obtained in this way.

Let $p_{1}, \ldots, p_{r}$ be the vertices of the polygon $P$. We may assume that $p_{i}=$ $H_{i} \cap H_{i+1}$, where $H_{r+1}=H_{1}$. Let $m_{i}=m_{i i+1}$. Since

$$
\sum_{i=1}^{r}\left(\pi / m_{i}\right)=(r-2) \pi
$$

we have

$$
\sum_{i=1}^{r} \frac{1}{m_{i}}=r-2 .
$$

The only solutions for $\left(r ; m_{1}, \ldots, m_{r}\right)$ are

$$
(3 ; 2,3,6), \quad(3 ; 2,4,4), \quad(3 ; 3,3,3), \quad(4 ; 2,2,2,2) .
$$




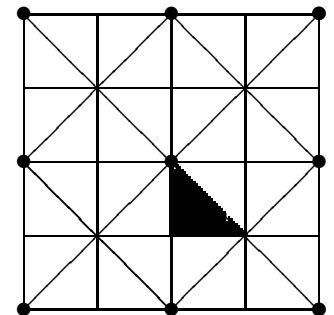

$(3 ; 2,4,4), G \cong \mathbb{Z}^{2} \rtimes D_{8}$

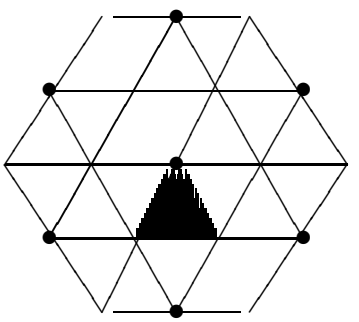

$(3 ; 3,3,3), G \cong \mathbb{Z}^{2} \rtimes S_{3}$

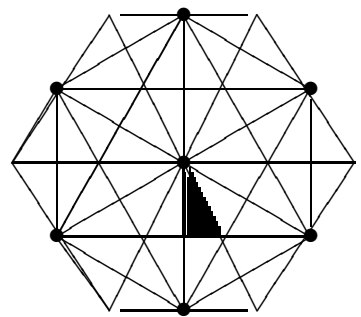

$(3 ; 2,3,6), G \cong \mathbb{Z}^{2} \rtimes D_{12}$

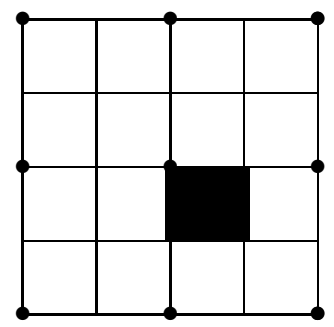

$(4 ; 2,2,2,2), G \cong \mathbb{Z}^{2} \rtimes D_{4}$

Figure 5

2.2. Spaces of constant curvature. The usual euclidean plane is an example of a 2-dimensional space of zero constant curvature. Recall that a space of constant curvature is a simply connected Riemannian homogeneous space $X$ such that the isotropy subgroup of its group of isometries $\operatorname{Iso}(X)$ at each point coincides with the full orthogonal group of the tangent space. Up to isometry and rescaling the metric, there are three spaces of constant curvature of fixed dimension $n$.

- The euclidean space $E^{n}$ with $\operatorname{Iso}(X)$ equal to the affine orthogonal group $\mathrm{AO}^{n}=\mathbb{R}^{n} \rtimes \mathrm{O}(n)$.

- The $n$-dimensional sphere

$$
S^{n}=\left\{\left(x_{0}, \ldots, x_{n}\right) \in \mathbb{R}^{n+1}: x_{0}^{2}+\ldots+x_{n}^{2}=1\right\}
$$

with $\operatorname{Iso}(X)$ equal to the orthogonal group $\mathrm{O}(n+1)$.

- The hyperbolic (or Lobachevsky) space

$$
H^{n}=\left\{\left(x_{0}, \ldots, x_{n}\right) \in \mathbb{R}^{n+1},-x_{0}^{2}+x_{1}^{2}+\ldots+x_{n}^{2}=-1, x_{0}>0\right\}
$$

with $\operatorname{Iso}(X)$ equal to the subgroup $\mathrm{O}(n, 1)^{+}$of index 2 of the orthogonal group $\mathrm{O}(n, 1)$ which consists of transformations of spinor norm 1 , that is, transformations that can be written as a product of reflections in vectors with positive norm. The Riemannian metric is induced by the hyperbolic metric in $\mathbb{R}^{n+1}$,

$$
d s^{2}=-d x_{0}^{2}+d x_{1}^{2}+\ldots+d x_{n}^{2} .
$$

We will be using a projective model of $H^{n}$, considering $H^{n}$ as the image of the subset

$$
C=\left\{\left(x_{0}, \ldots, x_{n}\right) \in \mathbb{R}^{n+1},-x_{0}^{2}+x_{1}^{2}+\ldots+x_{n}^{2}<0\right\}
$$


in the projective space $\mathbb{P}^{n}(\mathbb{R})$. The isometry group of the projective model is naturally identified with the group $\mathrm{PO}(n, 1)$. By choosing a representative of a point from $H^{n}$ with $x_{0}=1$, we can identify $H^{n}$ with the real ball

$$
K^{n}:\left\{\left(x_{1}, \ldots, x_{n}\right):|x|^{2}=x_{1}^{2}+\ldots+x_{n}^{2}<1\right\}
$$

(the Klein model). The metric is given by

$$
d s^{2}=\frac{1}{1-|x|^{2}} \sum_{i=1}^{n} d x_{i}^{2}+\frac{1}{\left(1-|x|^{2}\right)^{2}}\left(\sum_{i=1}^{n} x_{i} d x_{i}\right)^{2} .
$$

The closure of $H^{n}$ in $\mathbb{P}^{n}(\mathbb{R})$ is equal to the image of the set

$$
\bar{C}=\left\{\left(x_{0}, \ldots, x_{n}\right) \in \mathbb{R}^{n+1},-x_{0}^{2}+x_{1}^{2}+\ldots+x_{n}^{2} \leq 0\right\}
$$

in $\mathbb{P}^{n}(\mathbb{R})$. The boundary is called the absolute.

One defines the notion of a hyperplane in a space of constant curvature. If $X=E^{n}$, a hyperplane is an affine hyperplane. If $X=S^{n}$, a hyperplane is the intersection of $S^{n}$ with a linear hyperplane in $\mathbb{R}^{n+1}$ (a great circle when $n=2$ ). If $X=H^{n}$, a hyperplane is the nonempty intersection of $H^{n}$ with a projective hyperplane in $\mathbb{P}^{n}(\mathbb{R})$.

Each hyperplane $H$ in $E^{n}$ is a translate $a+L=\{x+a, x \in L\}$ of a unique linear hyperplane $\tilde{H}$ in the corresponding standard euclidean space $V=\mathbb{R}^{n}$. If $X^{n}=S^{n}$ or $H^{n}$, then a hyperplane $H$ is uniquely defined by a linear hyperplane $\tilde{H}$ in $V=\mathbb{R}^{n+1}$ equipped with the standard symmetric bilinear form of Sylvester signature $\left(t_{+}, t_{-}\right)=(n+1,0)$ or $(n, 1)$.

Any point $x \in V$ can be written uniquely in the form

$$
x=h+v,
$$

where $h \in H$ and $v \in V$ is orthogonal to $\tilde{H}$. We define a reflection with mirror hyperplane $H$ by the formula

$$
r_{H}(x)=h-v .
$$

One can also give a uniform definition of a hyperplane in a space of constant curvature as a totally geodesic hypersurface and define a reflection in such a space as an isometric involution whose set of fixed points is a hyperplane.

Let $H$ be a hyperplane in $X^{n}$. Its complement $X^{n} \backslash H$ consists of two connected components. The closure of a component is called a halfspace. A reflection $r_{H}$ permutes the two halfspaces. One can distinguish the two halfspaces by a choice of one of the two unit vectors in $V$ orthogonal to the corresponding linear hyperplane $\tilde{H}$. We choose it so that it belongs to the corresponding halfspace. For any vector $v$ perpendicular to a hyperplane in $H^{n}$ we have $(v, v)>0$ (otherwise the intersection of the hyperplane with $H^{n}$ is empty).

Let $H_{1}^{+}, H_{2}^{+}$be two halfspaces, and $e_{1}, e_{2}$ be the corresponding unit vectors. If $X \neq H^{n}$, the angle $\phi=\angle\left(H_{1}^{+}, H_{2}^{+}\right)=\angle\left(H_{1}^{-}, H_{2}^{-}\right)$is defined by

$$
\cos \phi=-\left(e_{1}, e_{2}\right), \quad 0 \leq \phi \leq \pi .
$$

If $X^{n}=H^{n}$ we use the same definition if $\left(e_{1}, e_{2}\right) \leq 1$; otherwise we say that the angle is divergent (in this case $\left(e_{1}, e_{2}\right)$ is equal to the hyperbolic cosine of the distance between the hyperplanes). 


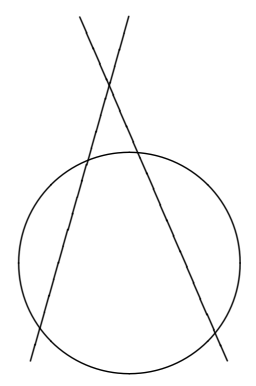

divergent

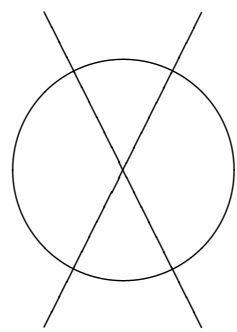

angle

\section{FiguRE 6}

A convex polytope in $X^{n}$ is a nonempty intersection of a locally finite ${ }^{1}$ set of halfspaces

$$
P=\cap_{i \in I} H_{i}^{-} .
$$

The normal vectors $e_{i}$ defining the halfspaces $H_{i}^{-}$all look inside the polytope. The hyperplanes $H_{i}$ 's are called faces of the polytope. In the case $X^{n}=H^{n}$ we also add to $P$ the points of the intersection lying on the absolute. We will always assume that no $e_{i}$ is a positive linear combination of others or, equivalently, none of the halfspaces contains the intersection of the rest of the halfspaces. In this case the set of bounding hyperplanes can be reconstructed from $P$.

A convex polytope has a finite volume if and only if it is equal to a convex hull of finitely many points (vertices) from $X^{n}$ (or from the absolute if $X^{n}=H^{n}$ ). Such a polytope has finitely many faces. If $X^{n}=E^{n}$ or $S^{n}$, it is a compact polytope. A polytope of finite volume in $H^{n}$ is compact only if its vertices do not lie on the absolute.

2.3. Reflection groups. A reflection group in a space of constant curvature is a discrete group of motions of $X^{n}$ generated by reflections.

Theorem 2.1. Let $\Gamma$ be a reflection group in $X^{n}$. There exists a convex polytope $P(\Gamma)=\cap_{i \in I} H_{i}^{-}$such that

(i) $P$ is a fundamental domain for the action of $\Gamma$ in $X^{n}$;

(ii) the angle between any two halfspaces $H_{i}^{-}, H_{j}^{-}$is equal to zero or $\pi / m_{i j}$ for some positive integer $m_{i j}$ unless the angle is divergent;

(iii) $\Gamma$ is generated by reflections $r_{H_{i}}, i \in I$.

Conversely, for every convex polytope $P$ satisfying property (ii) the group $\Gamma(P)$ generated by the reflections into its facets is a reflection group and $P$ satisfies (i).

Proof. Consider the set $\mathcal{H}$ of mirror hyperplanes of all reflections contained in $\Gamma$. For any mirror hyperplane $H$ and $g \in \Gamma$, the hyperplane $g(H)$ is the mirror hyperplane for the reflection $g r_{H} g^{-1}$. Thus the set $\mathcal{H}$ is invariant with respect to $\Gamma$. Let $K$ be a compact subset of $X^{n}$. For any hyperplane $H \in \mathcal{H}$ meeting $K$, we have $r_{H}(K) \cap K \neq \emptyset$. Since $\Gamma$ is a discrete group, the set $\{g \in G: g(K) \cap K \neq \emptyset\}$

\footnotetext{
${ }^{1}$ Locally finite means that each compact subset of $X^{n}$ is intersected by only finitely many hyperplanes.
} 
is finite. This shows that the set $\mathcal{H}$ is locally finite. The closure of a connected component of

$$
X^{n} \backslash \bigcup_{H \in \mathcal{H}} H
$$

is a convex polytope called a cell (or $\Gamma$-cell or a fundamental polyhedron) of $\Gamma$. Its faces are called walls. Two cells which share a common wall are called adjacent. The corresponding reflection switches the adjacent cells. This easily shows that the group $\Gamma$ permutes transitively the cells. Also it shows that any hyperplane from $\mathcal{H}$ is the image of a wall of $P$ under an element of the group $\Gamma(P)$ generated by the reflections with respect to walls of $P$. Thus $\Gamma=\Gamma(P)$. It is clear that the orbit of each point intersects a fixed cell $P$. The proof that no two interior points of $P$ belong to the same orbit follows from the last assertion of the theorem. Its proof is rather complicated, and we omit it (see [120], Chapter V, Theorem 1.2).

Let $H, H^{\prime}$ be two hyperplanes bounding $P$ for which the angle $\angle\left(H^{-}, H^{\prime-}\right)$ is defined and is not zero. The corresponding unit vectors $e, e^{\prime}$ span a plane in the vector space $V$ associated to $X^{n}$, and the restriction of the symmetric bilinear form to the plane is positive definite. The subgroup of $\Gamma$ generated by the reflections $r_{H}, r_{H^{\prime}}$ defines a reflection subgroup in $\Pi$. Thus the angle must be of the form $r \pi$ for some rational number $r$. If $r$ is not of the form $1 / m$ for some integer $m$, then $\Gamma$ contains a reflection with respect to a hyperplane intersecting the interior of $P$. By definition of $P$ this is impossible. This proves (i)-(iii).

Define a Coxeter polytope to be a convex polytope $P$ in which any two faces are either divergent or form the angle equal to zero or $\pi / m$ for some positive integer $m$ (or $\infty)$. Let $\left(e_{i}\right)_{\in I}$ be the set of unit vectors corresponding to the halfspaces $H_{i}^{-}$ defining $P$. The matrix

$$
G(P)=\left(\left(e_{i}, e_{j}\right)\right)_{(i, j) \in I \times I}
$$

is the Gram matrix of $P$, and its rank is the rank of the Coxeter polytope. The polytope $P$ is called irreducible if its Gram matrix is not equal to the nontrivial direct sum of matrices.

One can describe the matrix $G(P)$ via a certain labeled graph, the Coxeter diagram of $P$. Its vertices correspond to the walls of $P$. Two vertices corresponding to the hyperplanes with angle of the form $\pi / m, m \geq 3$, are joined with an edge labeled with the number $m-2$ (dropped if $m=3$ ) or joined with $m-2$ nonlabeled edges. Two vertices corresponding to parallel hyperplanes (i.e. forming the zero angle) are joined by a thick edge or an edge labeled with $\infty$. Two vertices corresponding to divergent hyperplanes are joined by a dotted edge.

Obviously an irreducible polytope is characterized by the condition that its Coxeter diagram is a connected graph.

Let $\Gamma$ be a reflection group in $X^{n}$ and $P$ be a $\Gamma$-cell. We apply the previous terminology concerning $P$ to $\Gamma$. Since $\Gamma$-cells are transitively permuted by $\Gamma$, the isomorphism type of the Gram matrix does not depend on a choice of $P$. In particular, we can speak about irreducible reflection groups. They correspond to Gram matrices which cannot be written as a nontrivial direct sum of their submatrices.

Suppose all elements of $\Gamma$ fix a point $x_{0}$ in $X^{n}$ (or on the absolute of $H^{n}$ ). Then all mirror hyperplanes of reflections in $\Gamma$ contain $x_{0}$. Therefore each $\Gamma$-cell is a polyhedral cone with vertex at $x_{0}$. In the case when $X^{n}=E^{n}$, we can use $x_{0}$ to identify $E^{n}$ with its linear space $V$ and the group $\Gamma$ with a reflection group in $S^{n-1}$. The same is true if $X^{n}=S^{n}$. If $X^{n}=H^{n}$ and $x_{0} \in H^{n}$ (resp. $x_{0}$ lies on the 
absolute), then by considering the orthogonal subspace in $\mathbb{R}^{n+1}$ to the line defined by $x_{0}$ we find an isomorphism from $\Gamma$ to a reflection group in $S^{n-1}$ (resp. $E^{n-1}$ ).

Any convex polytope of finite volume in $E^{n}$ or $H^{n}$ is nondegenerate in the sense that its faces do not have a common point and the unit norm vectors of the faces span the vector space $V$. A spherical convex polytope is nondegenerate if it does not contain opposite vertices.

Theorem 2.2. Let $\Gamma$ be an irreducible reflection group in $X^{n}$ with nondegenerate $\Gamma$-cell $P$. If $X^{n}=S^{n}$, then $\Gamma$ is finite and $P$ is equal to the intersection of $S^{n}$ with a simplicial cone in $\mathbb{R}^{n+1}$. If $X^{n}=E^{n}$, then $\Gamma$ is infinite and $P$ is a simplex in $E^{n}$.

This follows from the following simple lemma ([14], Chapter V, §3, Lemma 5):

Lemma 2.3. Let $V$ be a real vector space with positive definite symmetric bilinear form $(v, w)$ and let $\left(v_{i}\right)_{i \in I}$ be vectors in $V$ with $\left(v_{i}, v_{j}\right) \leq 0$ for $i \neq j$. Assume that the set $I$ cannot be nontrivially split into the union of two subsets $I_{1}$ and $I_{2}$ such that $\left(v_{i}, v_{j}\right)=0$ for $i \in I_{1}, j \in I_{2}$. Then the vectors $v_{i}$ are either linearly independent or span a hyperplane and a linear dependence can be chosen of the form $\sum a_{i} v_{i}$ with all $a_{i}$ positive.

The classification of irreducible nondegenerate Coxeter polytopes, and hence irreducible reflection groups $\Gamma$ in $S^{n}$ and $\mathbb{R}^{n}$ with nondegenerate $\Gamma$-cell, was given by Coxeter [27]. The corresponding list of Coxeter diagrams is given in Table 1 .

Here the number of nodes in the spherical (resp. euclidean) diagram is equal to the subscript $n$ (resp. $n+1$ ) in the notation. The number $n$ is equal to the rank of the corresponding Coxeter polytope. We will refer to diagrams from the first (resp. second) column as elliptic Coxeter diagrams (resp. parabolic Coxeter diagrams) of rank $n$.

Our classification of plane reflection groups in section 1.1 fits in this classification:

$$
(2,4,4) \longleftrightarrow \tilde{C}_{2},(2,3,6) \longleftrightarrow \tilde{G}_{2},(3,3,3) \longleftrightarrow \tilde{A}_{2},(2,2,2,2) \longleftrightarrow \tilde{A}_{1} \times \tilde{A}_{1}
$$

The list of finite reflection groups not of type $H_{3}, H_{4}, I_{2}(m), m \neq 6\left(I_{2}(6)\right.$ is often denoted by $G_{2}$ ) coincides with the list of Weyl groups of simple Lie algebras of the corresponding type $A_{n}, B_{n}$ or $C_{n}, G_{2}, F_{4}, E_{6}, E_{7}, E_{8}$. The corresponding Coxeter diagrams coincide with the Dynkin diagrams only in the cases $A, D$, and $E$. The second column corresponds to affine Weyl groups.

The group of type $A_{n}$ is the symmetric group $\Sigma_{n+1}$. It acts in the space

$$
V=\left\{\left(a_{1}, \ldots, a_{n+1}\right) \in \mathbb{R}^{n+1}: a_{1}+\ldots a_{n+1}=0\right\}
$$

with the standard inner product as the group generated by reflections in vectors $e_{i}-e_{i+1}, i=1, \ldots n$.

The group of type $B_{n}$ is isomorphic to the semi-direct product $2^{n} \rtimes \Sigma_{n}$. It acts in the euclidean space $\mathbb{R}^{n}$ as a group generated by reflections in vectors $e_{i}-e_{i+1}, i=$ $1, \ldots, n-1$, and $e_{n}$.

The group of type $D_{n}$ is isomorphic to the semi-direct product $2^{n-1} \rtimes \Sigma_{n}$. It acts in the euclidean space $\mathbb{R}^{n}$ as a group generated by reflections in vectors $e_{i}-e_{i+1}, i=$ $1, \ldots, n-1$, and $e_{n-1}+e_{n}$. 
TABLE 1. Spherical and euclidean real reflection groups

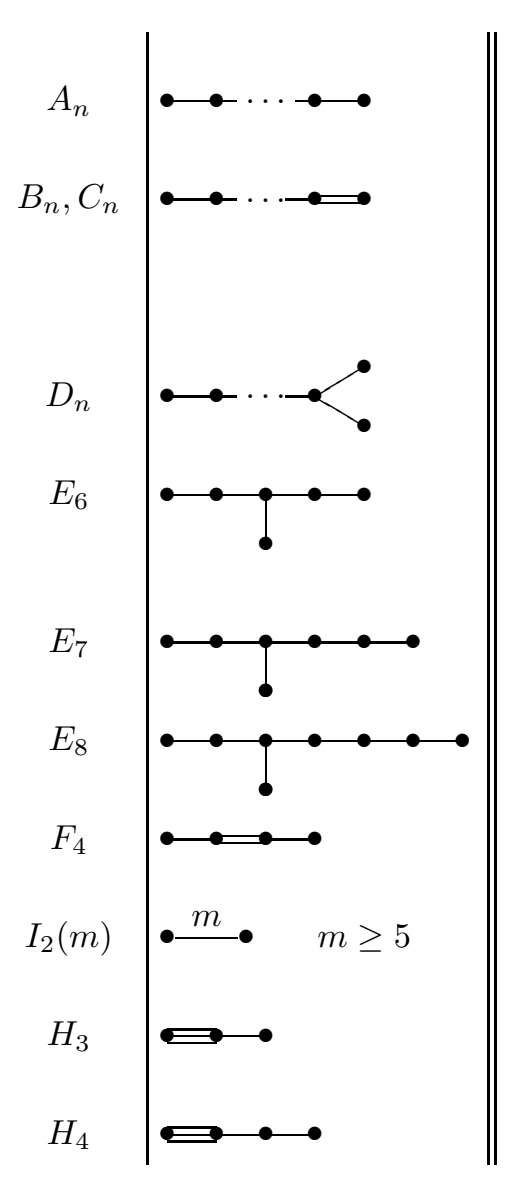

Spherical groups

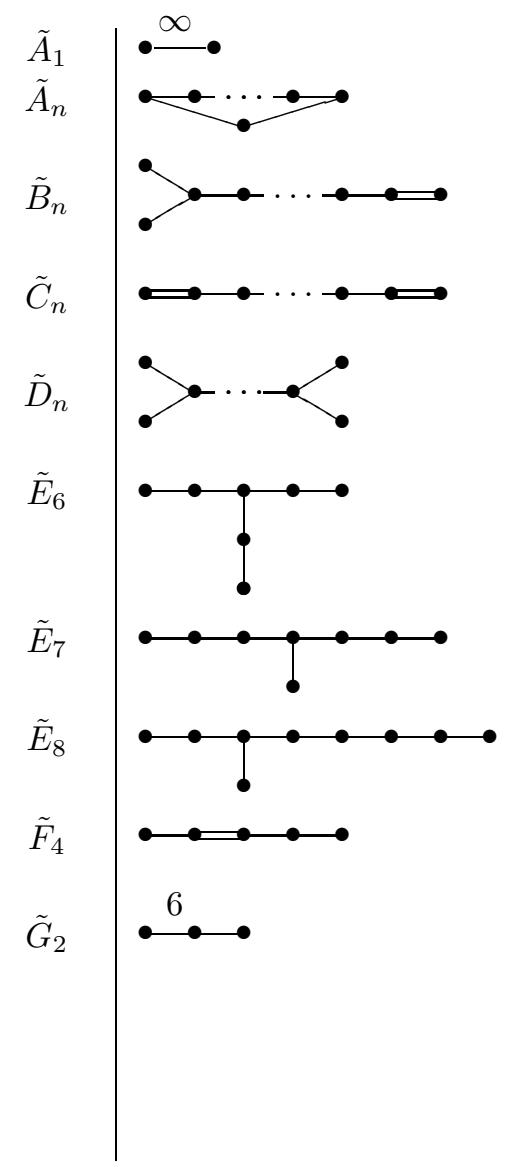

Euclidean groups

A discrete group $\Gamma$ of motions in $X^{n}$ admitting a fundamental domain of finite volume (resp. compact) is said to be of finite covolume (resp. cocompact). ${ }^{2}$ Obviously, a simplex in $S^{n}$ or $E^{n}$ is compact. Thus the previous list gives a classification of irreducible reflection groups of finite covolume in $E^{n}$ and $S^{n}$. They are are automatically cocompact.

The classification of reflection groups of finite covolume in $H^{n}$ is known only for $n=2$ (H. Poincaré) and $n=3$ (E. Andreev [5]). It is known that they do not exist if $n \geq 996$ ([65], [66], [95]) and even if $n>300$ (see the announcement in [87]). There are no cocompact reflection groups in $H^{n}$ for $n \geq 30$ [116].

One can give the following description of Coxeter diagrams defining reflection groups of cofinite volume (see [112]).

Proposition 2.4. A reflection group $\Gamma$ in $H^{n}$ is of finite covolume if and only if any elliptic subdiagram of rank $n-1$ of its Coxeter diagram can be extended in

\footnotetext{
${ }^{2}$ If $\Gamma$ is realized as a discrete subgroup of a Lie group that acts properly and transitively on $X^{n}$, then this terminology agrees with the terminology of discrete subgroups of a Lie group.
} 
exactly two ways to an elliptic subdiagram of rank $n$ or a parabolic subdiagram of rank $n-1$. Moreover, $\Gamma$ is cocompact if the same is true but there are no parabolic subdiagrams of rank $n-1$.

The geometric content of this proposition is as follows. The intersection of hyperplanes defining an elliptic subdiagram of rank $n-1$ is of dimension 1 (a onedimensional facet of the polytope). An elliptic subdiagram (resp. parabolic) of rank $n$ defines a proper (resp. improper) vertex of the polytope. So, the proposition says that $\Gamma$ is of finite covolume if and only if each one-dimensional facet joins precisely two vertices, proper or improper.

2.4. Coxeter groups. Recall that a Coxeter group is a group $W$ admitting an ordered set of generators $S$ of order 2 with defining relations

$$
\left(s s^{\prime}\right)^{m\left(s, s^{\prime}\right)}=1, s, s^{\prime} \in S,
$$

where $m\left(s, s^{\prime}\right)$ is the order of the product $s s^{\prime}$ (the symbol $\infty$ if the order is infinite). The pair $(W, S)$ is called a Coxeter system.

The Coxeter graph of $(W, S)$ is the graph whose vertices correspond to $S$ and any two different vertices are connected by $m\left(s, s^{\prime}\right)-2$ edges or by an edge labeled with $m\left(s, s^{\prime}\right)-2$ or a thick edge if $m\left(s, s^{\prime}\right)=\infty$. We say that $(W, S)$ is irreducible if the Coxeter graph is connected.

One proves the following theorem (see [112]).

Theorem 2.5. Let $P$ be a nondegenerate Coxeter polytope of finite volume in $X^{n}$ and $\Gamma(P)$ be the corresponding reflection group. The pair $(\Gamma(P), S)$, where $S$ is the set of reflections with respect to the set of faces of $P$ is a Coxeter system. Its Coxeter graph is equal to the Coxeter diagram of $P$, and the Gram matrix of $P$ is equal to the matrix

$$
\left(-\cos \frac{\pi}{m\left(s, s^{\prime}\right)}\right)_{\left(s, s^{\prime}\right) \in S \times S} .
$$

The converse is partially true. The following facts can be found in [14]. Let $(W, S)$ be an irreducible Coxeter system with no $m\left(s, s^{\prime}\right)$ equal to $\infty$. One considers the linear space $V=\mathbb{R}^{S}$ and equips it with a symmetric bilinear form $B$ defined by

$$
B\left(e_{s}, e_{s^{\prime}}\right)=-\cos \frac{\pi}{m\left(s, s^{\prime}\right)} .
$$

Assume that $B$ is positive definite $((W, S)$ is elliptic). Then $W$ is finite and isomorphic to a reflection group $\Gamma$ in the spherical space $S^{n}$, where $n+1=\# S-1$. The corresponding $\Gamma$-cell can be taken as the intersection of the sphere with the simplex in $\mathbb{R}^{n+1}$ with facets orthogonal to the vectors $e_{s}$.

Assume that $B$ is degenerate and semipositive definite $((W, S)$ is parabolic $)$. Then its radical $V_{0}$ is one-dimensional and is spanned by a unique vector $v_{0}=$ $\sum a_{s} e_{s}$ satisfying $a_{s}>0$ for all $s$ and $\sum a_{s}=1$. The group $W$ acts naturally as a reflection group $\Gamma$ in the affine subspace $E^{n}=\left\{\phi \in V^{*}: \phi\left(v_{0}\right)=1\right\}$ with the associated linear space $\left(V / V_{0}\right)^{*}$. The corresponding $\Gamma$-cell has $n+1$ facets orthogonal to the vectors $e_{s}$ and is a simplex in affine space.

Assume that $B$ is nondegenerate, indefinite and $W$ is of cofinite volume in the orthogonal group of $B((W, S)$ is hyperbolic). In this case the signature of $B$ is equal to $(n-1,1)$ and $C=\sum_{s \in S} \mathbb{R}_{+} e_{s}$ is contained in one of the two connected components of the set $\{x \in E: B(x, x)<0\}$. Let $H^{n}$ be the hyperbolic space equal 
to the image of this component in $\mathbb{P}(E)$. Then the action of $W$ in $H^{n}$ is isomorphic to a reflection group $\Gamma$. A $\Gamma$-cell can be chosen to be the image of the closure $\bar{C}$ of $C$ in $\mathbb{P}(E)$.

In all cases the Coxeter diagram of a $\Gamma$-cell coincides with the Coxeter graph of $(W, S)$. We will call the matrix given by $(2.3)$ the Gram matrix of $(W, S)$.

Remark 2.6. Irreducible reflection groups in $S^{n}$ and $E^{n}$ correspond to elliptic or parabolic irreducible Coxeter systems. Hyperbolic Coxeter systems define Coxeter simplices in $H^{n}$ of finite volume. Their Coxeter diagrams are called quasi-Lanner [120] or hyperbolic [14]. They are characterized by the condition that each of its proper subdiagrams is either elliptic or parabolic. If no parabolic subdiagram is present, then the simplex is compact and the diagram is Lanner or compact hyperbolic. The complete list of hyperbolic Coxeter diagrams can be found in [120] or [58].

Example 2.7. Let $W(p, q, r), 1 \leq p \leq q \leq r$, be the Coxeter group with Coxeter graph of type $T_{p, q, r}$ given in Figure 7 .

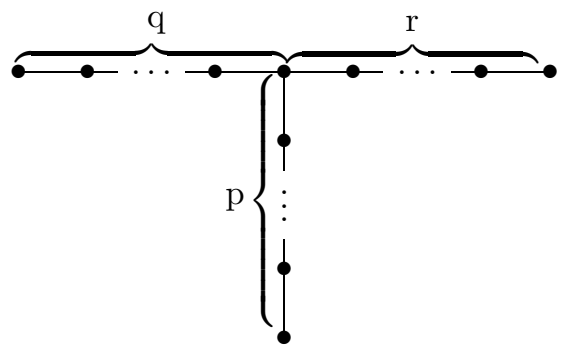

FiguRE 7

Then

- $W(p, q, r)$ is of elliptic type if and only if

$$
(p, q, r)=(1, q, r),(2,2, r),(2,3,3),(2,3,4),(2,3,5) ;
$$

- $W(p, q, r)$ is of parabolic type if and only if

$$
(p, q, r)=(2,4,4),(2,3,6),(3,3,3) ;
$$

- $W(p, q, r)$ is of hyperbolic type if and only if

$$
(p, q, r)=(3,4,4),(2,4,5),(2,3,7) .
$$

\section{LiNEAR REFLECTION GROUPS}

3.1. Pseudo-reflections. Let $E$ be a vector space over any field $K$. A pseudoreflection in $E$ is a linear invertible transformation $s: E \rightarrow E$ of finite order greater than 1 which fixes pointwise a hyperplane. A reflection is a diagonalizable pseudoreflection. A pseudo-reflection is a reflection if and only if its order is coprime to the characteristic of $K$.

Let $v$ be an eigenvector of a reflection $s$ of order $d$. Its eigenvalue $\eta$ different from 1 is a $d$ th root of unity $\eta \neq 1$. We can write $s$ in the form

$$
s(x)=x-\ell(x) v
$$


for some linear function $\ell: E \rightarrow K$. Its zeroes define the hyperplane of fixed points of $s$, the reflection hyperplane. Taking $x=v$ we obtain $\ell(v)=1-\eta$. This determines $\ell$ uniquely when $v$ is fixed; we denote it by $\ell_{v}$.

A pseudo-reflection (reflection) subgroup of $\mathrm{GL}(E)$ is a subgroup generated by pseudo-reflections (reflections).

Assume that we are given an automorphism $\sigma$ of $K$ whose square is the identity. We denote its value on an element $\lambda \in K$ by $\bar{\lambda}$. Let $B: E \times E \rightarrow K$ be a $\sigma$-hermitian form on $E$; i.e. $B$ is $K$-linear in the first variable and satisfies $B(x, y)=\overline{B(y, x)}$.

Let $\mathrm{U}(E, B, \sigma)$ be the unitary group of $B$, i.e. the subgroup of $K$-linear transformations of $E$ which preserve $B$. A pseudo-reflection subgroup in $\operatorname{GL}(E)$ is a unitary pseudo-reflection group if it is contained in a unitary group $\mathrm{U}(E, B, \sigma)$ for some $\sigma, B$ and for any reflection (3.1) one can choose a vector $v$ with $B(v, v) \neq 0$.

The additional condition implies that

$$
\ell_{v}(x)=\frac{(1-\eta) B(x, v)}{B(v, v)} \text { for all } x \in V .
$$

In particular, the vector $v$ is orthogonal to the hyperplane $\ell_{v}^{-1}(0)$.

Finite reflection groups are characterized by the following property of its algebra of invariant polynomials ([14], Chapter V, $\S 5$, Theorem 4).

Theorem 3.1. A finite subgroup $G$ of $G L(E)$ of order prime to char $(K)$ is a reflection group if and only if the algebra $S(E)^{G}$ of invariants in the symmetric algebra of $E$ is isomorphic to a polynomial algebra.

In the case $K=\mathbb{C}$ this theorem was proven by Shephard and Todd [102], and in the case of arbitrary characteristic but for groups generated by reflections of order 2 , it was proven by C. Chevalley [19].

We will be concerned with the case where $K=\mathbb{R}$ and $\sigma=\operatorname{id}_{E}$ or $K=\mathbb{C}$ and $\sigma$ is the complex conjugation. In the real case a reflection is necessarily of order 2 .

Let $G$ be a finite reflection subgroup of $\operatorname{GL}(E)$. By taking some positive definite symmetric bilinear (resp. hermitian) form and averaging it, we see that $G$ is conjugate in $\mathrm{GL}(E)$ to a unitary reflection group. In fact, under isomorphism from $E$ to the standard euclidean (resp. unitary) space $\mathbb{R}^{n}$ (resp. $\mathbb{C}^{n}$ ), the group $G$ is isomorphic to a reflection subgroup of $\mathrm{O}(n)$ (resp. $\mathrm{U}(n)$ ). In the real case $G$ becomes isomorphic to a reflection group in $S^{n-1}$ and hence is isomorphic to the product of irreducible reflection spherical groups.

An example of an infinite real reflection group in $\mathbb{R}^{n+1}$ is a reflection group in the hyperbolic space $H^{n}$. It is an orthogonal reflection group with respect to a symmetric bilinear form of signature $(n, 1)$.

3.2. Finite complex linear reflection groups. They were classified by Shephard and Todd [102]. Table 2 gives the list of irreducible finite linear reflection groups (in the order given by Shephard-Todd; see also the table in [62], p. 166). The last column in the table gives the degrees of the generators of the algebra $S(E)^{G}$.

The group $G(m, p, n)$ is equal to the semi-direct product $A(m, n, p) \rtimes \Sigma_{n}$, where $A(m, n, p)$ is a diagonal group of $n \times n$-matrices with $m$ th roots of unity at the diagonal whose product is an $(m / p)$ th root of unity. The semi-direct product is defined with respect to the action of $\Sigma_{n}$ by permuting the columns of the matrices.

Groups 3-22 are some extensions of binary polyhedral groups (i.e. finite subgroups of $\mathrm{SL}(2, \mathbb{C})$ ). 
TABLE 2. Finite complex reflection groups

\begin{tabular}{|c|c|c|c|c|}
\hline Number & Name & Order & $\operatorname{dim} E$ & Degrees \\
\hline 1 & $A_{n}=\Sigma_{n+1}$ & $(n+1) !$ & $n$ & $2,3, \ldots, n+1$ \\
\hline 2 & $G(m, p, n)$ & $m^{n} n ! / p$ & $n$ & $m, m+1, \ldots,(n-1) m, m n / p$ \\
\hline 3 & {[]$^{m}$} & $m$ & 1 & $m$ \\
\hline 4 & $3[3] 3$ & 24 & 2 & 4,6 \\
\hline 5 & $3[4] 3$ & 72 & 2 & 6,12 \\
\hline 6 & $3[6] 2$ & 48 & 2 & 4,12 \\
\hline 7 & $<3,3,3>_{2}$ & 144 & 2 & 12,12 \\
\hline 8 & $4[3] 4$ & 96 & 2 & 8,12 \\
\hline 9 & $4[6] 2$ & 192 & 2 & 8,24 \\
\hline 10 & $4[4] 3$ & 288 & 2 & $\overline{12,24}$ \\
\hline 11 & $<4,3,2>_{12}$ & 576 & 2 & 24,24 \\
\hline 12 & $\mathrm{GL}(2,3)$ & 48 & 2 & 6,8 \\
\hline 13 & $<4,3,2>_{2}$ & 96 & 2 & 8,12 \\
\hline 14 & $3[8] 2$ & 144 & 2 & 6,24 \\
\hline 15 & $<4,3,2>_{6}$ & 288 & 2 & $\overline{12,24}$ \\
\hline 16 & $5[3] 5$ & 600 & 2 & 20,30 \\
\hline 17 & 5]6]2 & 1200 & 2 & 20,60 \\
\hline 18 & $5[4] 3$ & 1800 & 2 & 60,60 \\
\hline 19 & $<5,3,2>_{30}$ & 3600 & 2 & 60,60 \\
\hline 20 & $3[5] 3$ & 360 & 2 & 12,30 \\
\hline 21 & $3[10] 2$ & 720 & 2 & 12,60 \\
\hline 22 & $<5,3,2>_{2}$ & 240 & 2 & 12,20 \\
\hline 23 & $H_{3}$ & 120 & 3 & $2,6,10$ \\
\hline 24 & $J_{3}(4)$ & 336 & 3 & $4,6,14$ \\
\hline 25 & $L_{3}$ & 648 & 3 & $6,9,12$ \\
\hline 26 & $M_{3}$ & 1296 & 3 & $6,12,18$ \\
\hline 27 & $J_{3}(5)$ & 2160 & 3 & $6,12,30$ \\
\hline 28 & $F_{4}$ & 1152 & 4 & $2,6,8,12$ \\
\hline 29 & $\overline{N_{4}}$ & 7680 & 4 & $4,8,12,20$ \\
\hline 30 & $H_{4}$ & 14,440 & 4 & $2,12,20,30$ \\
\hline 31 & $E N_{4}$ & $64 \cdot 6 !$ & 4 & $8,12,20,24$ \\
\hline 32 & $L_{4}$ & $216 \cdot 6 !$ & 4 & $12,18,24,30$ \\
\hline 33 & $K_{5}$ & $72 \cdot 6 !$ & 5 & $4,6,10,12,18$ \\
\hline 34 & $K_{6}$ & $108 \cdot 9 !$ & 5 & $4,6,10,12,18$ \\
\hline 35 & $E_{6}$ & $72 \cdot 6 !$ & 6 & $2,5,6,8,9,12$ \\
\hline 36 & $E_{7}$ & $8 \cdot 9 !$ & 7 & $2,6,8,10,12,14,18$ \\
\hline 37 & $E_{8}$ & $192 \cdot 9 !$ & 8 & $2,8,12,14,18,20,24,30$ \\
\hline
\end{tabular}

All real spherical irreducible groups are in the list. We have seen already the groups of types $A_{n}, E_{6}, E_{7}, E_{8}, H_{3}, H_{4}, F_{4}$. The groups of type $B_{n}$ are the groups $G(2,1, n)$. The groups of type $D_{n}$ are the groups $G(2,2, n)$. Finally, the groups of type $I_{2}(m)$ are the groups $G(m, m, 2)$. These groups are distinguished from other groups by the property that one of invariant polynomials is of degree 2 . 
3.3. Complex crystallographic reflection groups. A complex analog of a space of constant curvature is a simply connected complex Kähler manifold of constant holomorphic curvature (complex space form). There are three types of such spaces (see [60]):

- $E_{\mathbb{C}}^{n}$, the $n$-dimensional affine space equipped with the standard hermitian form $|z|^{2}=\sum\left|z_{i}\right|^{2}$. It is a homogeneous space $\left(\mathbb{C}^{n} \rtimes \mathrm{U}(n)\right) / \mathrm{U}(n)$;

- $\mathbb{P}^{n}(\mathbb{C})$, the $n$-dimensional complex projective space equipped with the standard Fubini-Study metric. It is a homogeneous space $\mathrm{PU}(n+1) / \mathrm{U}(n)$.

- $H_{\mathbb{C}}^{n}=\left\{z \in \mathbb{C}^{n}:|z|<1\right\}$, the $n$-dimensional complex hyperbolic space. The hermitian metric on $H_{\mathbb{C}}^{n}$ is defined by

$$
\left.\frac{1}{1-|z|^{2}}\left(\sum_{i=1}^{n} z_{i} d \bar{z}_{i}+\bar{z}_{i} d z_{i}\right)+\left(1-|z|^{2}\right) \sum_{i=1}^{n} d z_{i} d \bar{z}_{i}\right) .
$$

They are simply connected hermitian complex homogeneous manifolds of dimension $n$ with isotropy subgroups equal to the unitary group $\mathrm{U}(n)$.

The complex hyperbolic space has a model in complex projective space $\mathbb{P}^{n}(\mathbb{C})$ equal to the image of the subset

$$
C=\left\{\left(z_{0}, z_{1}, \ldots, z_{n}\right) \in \mathbb{C}^{n+1}:-\left|z_{0}\right|^{2}+\left|z_{1}\right|^{2}+\ldots+\left|z_{n}\right|^{2}<0\right\} .
$$

The unitary group $\mathrm{U}(n+1)$ of the hermitian form $-\left|z_{0}\right|^{2}+\left|z_{1}\right|^{2}+\ldots+\left|z_{n}\right|^{2}$ of signature $(n, 1)$ acts transitively on $H_{\mathbb{C}}^{n}$ with isotropy subgroup $\mathrm{U}(n)$. It defines a transitive action of $\mathrm{PU}(n, 1)=\mathrm{U}(n, 1) / \mathrm{U}(1)$ with isotropy subgroups isomorphic to $\mathrm{U}(n)$.

Let $X_{\mathbb{C}}^{n}$ be an $n$-dimensional complex space form. A reflection in $X_{\mathbb{C}}^{n}$ is a holomorphic isometry whose set of fixed points is a hypersurface. A reflection group is a discrete group of holomorphic automorphisms generated by reflections. A reflection group $\Gamma$ of $X_{\mathbb{C}}^{n}=\mathbb{P}^{n}(\mathbb{C})$ (resp. $H_{\mathbb{C}}^{n}$ ) can be centrally extended to a reflection subgroup of $\mathrm{U}(n)$ (resp. $\mathrm{U}(n, 1)$ ) such that over every reflection lies a linear reflection.

A reflection group of $E_{\mathbb{C}}^{n}$ is a discrete subgroup of $\mathbb{C}^{n} \rtimes \mathrm{U}(n)$ which is generated by affine reflections. It can be considered as a linear reflection group in a complex vector space $V$ of dimension $n+1$ equipped with a hermitian form of Sylvester signature $\left(t_{+}, t_{-}, t_{0}\right)=(n, 0,1)$. We take for $E_{\mathbb{C}}^{n}$ the affine subspace of the dual linear space $V^{*}$ of linear functions $\phi \in V^{*}$ satisfying $\phi(v)=1$, where $v$ is a fixed nonzero vector in $V^{\perp}$. The corresponding linear space is the hyperplane $\left\{\phi \in V^{*}\right.$ : $\phi(v)=0\}$.

A reflection group $\Gamma$ of cofinite volume in $E_{\mathbb{C}}^{n}$ or $H_{\mathbb{C}}^{n}$ is called a complex crystallographic group (affine, hyperbolic). If $X_{\mathbb{C}}^{n}=\mathbb{P}^{n}(\mathbb{C})$, it is a finite group defined by a finite linear complex reflection group in $\mathbb{C}^{n+1}$. If $X_{\mathbb{C}}^{n}=E_{\mathbb{C}}^{n}$, then $\Gamma$ leaves invariant a lattice $\Lambda \subset \mathbb{C}^{n}$ of rank $2 n$ (so that $\mathbb{C}^{n} / \Lambda$ is a compact complex $n$-torus) and $\operatorname{Lin}(\Gamma)$ is a finite subgroup of $\mathrm{U}(n)$. This implies that $\Gamma$ is also cocompact.

If $X_{\mathbb{C}}^{n}=\mathbb{P}^{n}(\mathbb{C})$, then $\Gamma$, being a discrete subgroup of a compact Lie group $\mathrm{PU}(n+1)$, is finite and cocompact.

There is a classification of crystallographic reflection groups in $E_{\mathbb{C}}^{n}$ (due to V. Popov [93]). ${ }^{3}$

First observe that any $a \in E_{\mathbb{C}}^{n}$ defines a surjective homomorphism $g \mapsto \bar{g}$ from the affine group to the linear group of the corresponding complex linear space $V$. We write any $x \in E_{\mathbb{C}}^{n}$ in the form $x=a+v$, for a unique $v \in V$, and get

\footnotetext{
${ }^{3}$ According to [50] some groups are missing in Popov's list.
} 
$g(a+v)=g(a)+\bar{g}(v)$. This definition of $\bar{g}$ does not depend on the choice of $a$. In particular, choosing $a$ on a reflecting affine hyperplane $H$, we see that $\bar{g}$ is a linear reflection which fixes $H-a$. This implies that the image of a crystallographic reflection subgroup $\Gamma$ of $\mathbb{C}^{n} \rtimes \mathrm{U}(n)$ is a finite reflection subgroup of $\mathrm{U}(n)$.

Theorem 3.2. Let $G$ be a finite irreducible reflection group in $\mathrm{U}(n)$. Then the following properties are equivalent:

(i) there exists a complex reflection group $\Gamma$ in $E_{\mathbb{C}}^{n}$ with linear part $G$;

(ii) there exists a $G$-invariant lattice $\Lambda \subset E_{\mathbb{C}}^{n}$ of rank $2 n$;

(iii) the number of the group $G$ in Table 2 is $1,2(m=2,3,4,6), 3(m=2,3,4,6)$, $4,5,8,12,24-29,31-37$.

If $G$ is not of type $G(4,2, n), n \geq 4$ (number 2), or $G L(2,3)$ (type 12) or $E N_{4}$ (number 31), ${ }^{4}$ then $\Gamma$ is equal to the semi-direct product $\Lambda \rtimes G$. In the exceptional cases, $\Gamma$ is either the semi-direct product or some nontrivial extension of $G$ with normal subgroup $\Lambda$.

A table in [93] describes all possible lattices and the extensions for each $G$ as above.

Recall from Theorem 3.1 that the algebra of invariant polynomials of a finite complex reflection group $\Gamma$ in $\mathbb{C}^{n}$ is a polynomial algebra. This can be restated as follows. One considers the induced action of $\Gamma$ in $\mathbb{P}^{n-1}(\mathbb{C})$ and the orbit space $\mathbb{P}^{n-1}(\mathbb{C}) / \Gamma$ which exists as a projective algebraic variety. Now the theorem asserts that this variety is isomorphic to a weighted projective space $\mathbb{P}\left(q_{1}, \ldots, q_{n}\right){ }^{5}$ where the weights are equal to the degrees of free generators of the invariant algebra. The following is an analog of Theorem 3.1 for affine complex crystallographic groups due to Bernstein-Shwarzman [11] and Looijenga [75].

Theorem 3.3. Assume that the linear part of a complex crystallographic group $\Gamma$ is a complexification of a real finite reflection group $W$. Then the orbit space $E_{\mathbb{C}}^{n} / \Gamma$ exists as an algebraic variety and is isomorphic to a weighted projective space $\mathbb{P}\left(q_{0}, \ldots, q_{n}\right)$, where the weights are explicitly determined by $W$.

It is conjectured that the same is true without additional assumption on the linear part.

Example 3.4. Let $G$ be a finite complex reflection group arising from the complexification of a real reflection group $G_{r}$. Any such group is realized as the linear part of a complex crystallographic group $\Gamma$ in affine space and $\Gamma$ is the semi-direct product of $G$ and a $G$-invariant lattice. Suppose $G_{r}$ is of $A D E$ type. Let $e_{1}, \ldots, e_{n}$ be the norm vectors of the Coxeter polytope in $\mathbb{R}^{n}$. For any $\tau=a+b i, b>0$, consider the lattice $\Lambda_{\tau}$ in $\mathbb{C}^{n}$ spanned by the vectors $e_{i}$ and $\tau e_{i}$. This is a $G$-invariant lattice and every $G$-invariant lattice is obtained in this way. Moreover $\Lambda_{\tau}=\Lambda_{\tau^{\prime}}$ if and only if $\tau$ and $\tau^{\prime}$ belong to the same orbit of the modular group $\operatorname{PSL}(2, \mathbb{Z})$ which acts on the upper halfplane $\{z=a+b i \in \mathbb{C}: b>0\}$ by the Möbius transformations $z \mapsto(a z+b) /(c z+d)$. The linear part $G$ is a finite group of automorphisms of the compex torus $\left(\mathbb{C} / \Lambda_{\tau}\right)^{n}$ with the orbit space $\left(\mathbb{C} / \Lambda_{\tau}\right)^{n} / G$ isomorphic to a weighted

\footnotetext{
${ }^{4}$ Also $G(6,6, n)$ as pointed out in [50].

${ }^{5}$ This is the quotient of $\mathbb{C}^{n} \backslash\{0\}$ by the action of $\mathbb{C}^{*}$ defined in coordinates by $\left(z_{1}, \ldots, z_{n}\right) \rightarrow$ $\left(\lambda^{q_{1}} z_{1}, \ldots, \lambda^{q_{n}} z_{n}\right)$.
} 
projective space. In the case when $G_{r}$ is the Weyl group of a simple simply connected Lie group $H$, this quotient is naturally isomorphic to the moduli space of principal $H$-bundles on the elliptic curve $\mathbb{C} / \mathbb{Z}+\tau \mathbb{Z}$ (see [44]).

Remark 3.5. If $\Gamma$ is a real crystallographic group in affine space $E^{n}$, then its complexification is a complex noncrystallographic reflection group in $E_{\mathbb{C}}^{n}$. Every complex noncrystallographic reflection group in $E_{\mathbb{C}}^{n}$ is obtained in this way (see [93], $2.2)$.

We will discuss later a construction of complex crystallographic reflection groups in $H_{\mathbb{C}}^{n}$ for $n \leq 9$. The largest known dimension $n$ for which such groups exist is 13 $([3])$. It is believed that these groups occur only in finitely many dimensions.

\section{Quadratic Lattices AND THEIR REFleCtion GROUPS}

4.1. Integral structure. Let $\Gamma$ be an orthogonal linear reflection group in a real vector space $V$ of dimension $n$ equipped with a nondegenerate symmetric bilinear form of signature $(n, 0)$ or $(n-1,1)$. We assume that the intersection of its reflection hyperplanes is the origin. We say that $\Gamma$ admits an integral structure if it leaves invariant a free abelian subgroup $M \subset V$ of rank $n$ generating $V$. In other words, there exists a basis $\left(e_{1}, \ldots, e_{n}\right)$ in $V$ such that $\sum_{i=1}^{n} \mathbb{Z} e_{i}$ is $\Gamma$-invariant.

A linear reflection group admitting an integral structure is obviously a discrete subgroup of the orthogonal group $O(V)$ and hence acts discretely on the corresponding space of constant curvature $S^{n-1}$ or $H^{n-1}$ (because the isotropy subgroups are compact subgroups of $O(V)$ ). Thus $\Gamma$ is a reflection group of $S^{n-1}$ or $H^{n-1}$. By a theorem of Siegel [104], the group $\mathrm{O}(M)=\{g \in O(V): g(M)=M\}$ is of finite covolume in the orthogonal group $\mathrm{O}(V)$; hence $\Gamma$ is of finite covolume if and only if it is of finite index in $\mathrm{O}(M)$.

Let $H \subset V$ be a reflection hyperplane in $\Gamma$ and let $e_{H}$ be an orthogonal vector to $H$. In the hyperbolic case we assume that $H$ defines a hyperplane in $H^{n-1}$; hence $\left(e_{H}, e_{H}\right)>0$. The reflection $r_{H}$ is defined by

$$
r_{H}(x)=x-\frac{2\left(x, \alpha_{H}\right)}{\left(\alpha_{H}, \alpha_{H}\right)} e_{H}
$$

for some vector $\alpha_{H}$ proportional to $e_{H}$. Taking $x$ from $M$ we obtain that the vector $\frac{2\left(x, \alpha_{H}\right)}{\left(\alpha_{H}, \alpha_{H}\right)} \alpha_{H}$ belongs to $M$. Replacing $\alpha_{H}$ by proportional vector, we may assume that $\alpha_{H} \in M$ and also that $\alpha_{H}$ is a primitive vector in $M$ (i.e. $M / \mathbb{Z} \alpha_{H}$ is torsion-free). We call such a vector a root vector associated to $H$. The root vectors corresponding to the faces of a fundamental polytope are called the fundamental root vectors.

A root vector is uniquely defined by $H$ up to multiplication by -1 . Using the primitivity property of root vectors it is easy to see that, for all $x \in M$,

$$
2(x, \alpha) \in(\alpha, \alpha) \mathbb{Z} .
$$

In particular, if $\alpha, \beta$ are not perpendicular root vectors, then $2(\alpha, \beta) /(\beta, \beta)$ and $2(\alpha, \beta) /(\alpha, \alpha)$ are nonzero integers, so that the ratio $(\beta, \beta) /(\alpha, \alpha)$ is a rational number. If the Coxeter diagram is connected, we can fix one of the roots $\alpha$ and multiply the quadratic form $(x, x)$ on $V$ by $(\alpha, \alpha)^{-1}$ to assume that $(\beta, \beta) \in \mathbb{Q}$ for all root vectors. This implies that the Gram matrix $\left(e_{i}, e_{j}\right)$ of a basis of $M$ has 
entries in $\mathbb{Q}$. Multiplying the quadratic form by an integer, we may assume that it is an integral matrix; hence

$$
(x, y) \in \mathbb{Z}, \text { for all } x, y \in M \text {. }
$$

Example 4.1. Let $\Gamma$ be an irreducible finite real reflection group whose Dynkin diagram does not contain multiple edges (i.e. of types $A, D, E)$. Let $\left(\alpha_{1}, \ldots, \alpha_{n}\right)$ be the unit norm vectors of its fundamental Coxeter polytope and let $M \subset V$ be the span of these vectors. If we multiply the inner product in $V$ by 2 , we obtain $\left(\alpha_{i}, \alpha_{j}\right)=-2 \cos \frac{\pi}{m_{i j}} \in\{0,2,-1\}$. Hence the reflections $r_{\alpha_{i}}: x \mapsto x-\left(x, \alpha_{i}\right) \alpha_{i}$ leave $M$ invariant. Thus $\Gamma$ admits an integral structure and the Gram matrix of its basis $\left(\alpha_{1}, \ldots, \alpha_{n}\right)$ is equal to twice the matrix $(2.2)$. Note that $\left(\alpha_{i}, \alpha_{i}\right)=2, i=$ $1, \ldots, n$.

Let $\Gamma$ be of type $B_{n}$ and $e_{s}, s=1, \ldots, n$, be the unit normal vectors defined by a fundamental polytope. We assume that $m(n, n-1)=m(n-1, n)=4$ and other $m\left(s, s^{\prime}\right)$ take values in $\{1,2,3\}$. Let $\alpha_{i}=e_{i}$ if $i \neq n$ and $\alpha_{n}=\sqrt{2} e_{n}$. It is easy to see now that $\frac{2\left(x, \alpha_{i}\right)}{\left(\alpha_{i}, \alpha_{i}\right)} \in \mathbb{Z}$ for any $x$ in the span $M$ of the $\alpha_{i}$ 's. This shows that $M$ defines an integral structure on $\Gamma$ and $(x, y) \in \mathbb{Z}$ for any $x, y \in M$. We have

$$
\left(\alpha_{i}, \alpha_{i}\right)= \begin{cases}1 & \text { if } i \neq n \\ 2 & \text { otherwise }\end{cases}
$$

We leave it to the reader to check that the groups of type $F_{4}$ and $G_{2}=I_{2}(6)$ also admit an integral structure. However, the remaining groups do not.

4.2. Quadratic lattices. A (quadratic) lattice is a free abelian group $M$ equipped with a symmetric bilinear form with values in $\mathbb{Z}$. The orthogonal group $\mathrm{O}(M)$ of a lattice is defined in the natural way as the subgroup of automorphisms of the abelian group preserving the symmetric bilinear form. More generally one defines in an obvious way an isometry or isomorphism of lattices.

Let $V$ be a real vector space equipped with a symmetric bilinear form $(x, y)$ and $\left(e_{i}\right)_{i \in I}$ be a basis in $V$ such that $\left(e_{i}, e_{j}\right) \in \mathbb{Z}$ for all $i, j \in I$. Then the $\mathbb{Z}$-span $M$ of the basis is equipped naturally with the structure of a quadratic lattice. We have already seen this construction in the beginning of the section. The orthogonal group $\mathrm{O}(M)$ coincides with the group introduced there. Obviously every quadratic lattice is obtained in this way by taking $V=M_{\mathbb{R}}=M \otimes_{\mathbb{Z}} \mathbb{R}$ and extending the bilinear form by linearity.

Recall some terminology in the theory of integral quadratic forms stated in terms of lattices. The signature of a lattice $M$ is the Sylvester signature $\left(t_{+}, t_{-}, t_{0}\right)$ of the corresponding real quadratic form on $V=M_{\mathbb{R}}$. We omit $t_{0}$ if it is equal to zero. A lattice with $t_{-}=t_{0}=0$ (resp. $t_{+}=t_{0}=0$ ) is called positive definite (resp. negative definite). A lattice $M$ of signature with $(1, a)$ or $(a, 1)$ where $a \neq 0$ is called hyperbolic (or Lorentzian).

All lattices are divided into two types: even if the values of its quadratic form are even and odd otherwise.

Assume that the lattice $M$ is nondegenerate; that is $t_{0}=0$. This ensures that the map

$$
\iota_{M}: M \rightarrow M^{*}=\operatorname{Hom}_{\mathbb{Z}}(M, \mathbb{Z}), \quad m \mapsto(m, ?)
$$


is injective. Since $M^{*}$ is an abelian group of the same rank as $M$, the quotient group

$$
D_{M}=M^{*} / \iota(M)
$$

is a finite group (the discriminant group of the lattice $M$ ). Its order $d_{M}$ is equal to the absolute value of the discriminant of $M$ defined as the determinant of a Gram matrix of the symmetric bilinear form of $M$. A lattice is called unimodular if the map (4.4) is bijective (equivalently, if its discriminant is equal to \pm 1 ).

Example 4.2. Let $M$ be the lattice defining an integral structure on a finite reflection group from Example 4.1. It is an even positive definite lattice for the groups of types $A, D, E$ and odd positive definite lattice for groups of type $B_{n}, F_{4}, G_{2}$. These lattices are called finite root lattices of the corresponding type.

Example 4.3. Let $\Gamma$ be an irreducible linear reflection group in $V$ admitting an integral structure $M$. It follows from (4.2) that, after rescaling the inner product in $V$, we may assume that $M$ is a lattice in $V$ with $M_{\mathbb{R}}=V$. For example, consider the group $\Gamma=W(p, q, r)$ from Example 2.7 as a linear reflection group in $\mathbb{R}^{n}$, where $n=p+q+r-2$. The unit vectors $e_{i}$ of a fundamental Coxeter polytope satisfy $\left(e_{i}, e_{j}\right)=-2 \cos \frac{\pi}{m_{i j}}$, where $m_{i j} \in\{1,2,3\}$. Thus, rescaling the quadratic form in $V$ by multiplying its values by 2 , we find fundamental root vectors $\alpha_{i}$ such that $\left(\alpha_{i}, \alpha_{j}\right) \in \mathbb{Z}$. The lattice $M$ generated by these vectors defines an integral structure of $\Gamma$. The Gram matrix $G$ of the set of fundamental root vectors has 2 at the diagonal, and $2 I_{n}-G$ is the incidence matrix of the Coxeter graph of type $T_{p, q, r}$ from Example 2.7. We denote the lattice $M$ by $E_{p, q, r}$. One computes directly the signature of $M$ to obtain that $E_{p, q, r}$ is nondegenerate and positive definite if and only if $\Gamma$ is a finite reflection group of type $A, D, E\left(r=1\left(A_{n}\right)\right.$ or $r=p=2\left(D_{n}\right)$ or $\left.r=2, p=3, q=3,4,5\left(E_{6}, E_{7}, E_{8}\right)\right)$.

The lattice $E_{p, q, r}$ is degenerate if and only if it corresponds to a parabolic reflection group of type $\tilde{E}_{6}, \tilde{E}_{7}, \tilde{E}_{8}$. The lattice $E_{p, q, r}^{\perp}$ is of rank 1 and $E_{p, q, r} / E_{p, q, r}^{\perp}$ is isomorphic to the lattice $E_{p-1, q, r}, E_{p, q-1, r}, E_{p, q, r-1}$, respectively.

In the remaining cases $E_{p, q, r}$ is a hyperbolic lattice of signature $(n-1,1)$.

It is also easy to compute the determinant of the Gram matrix to obtain that the absolute value of the discriminant of a nondegenerate lattice $E_{p, q, r}$ is equal to $|p q r-p q-p r-q r|$. In particular, it is a unimodular lattice if and only if $(p, q, r)=(2,3,5)$ or $(2,3,7)$.

One can also compute the discriminant group of a nondegenerate lattice $E_{p, q, r}$ (see [17]).

Every subgroup of $M$ is considered as a lattice with respect to the restriction of the quadratic form (sublattice). The orthogonal complement of a subset $S$ of a lattice is defined to be the set of vectors $x$ in $M$ such that $(x, s)=0$ for all $s \in S$. It is a primitive sublattice of $M$ (i.e. a subgroup of $M$ such that the quotient group is torsion-free). Also one naturally defines the orthogonal direct sum $M \perp N$ of two (and finitely many) lattices.

The lattice $M$ of rank 2 defined by the matrix $\left(\begin{array}{ll}0 & 1 \\ 1 & 0\end{array}\right)$ is denoted by $U$ and is called the hyperbolic plane.

For any lattice $M$ and an integer $k$ we denote by $M(k)$ the lattice obtained from $M$ by multiplying its quadratic form by $k$. For any integer $k$ we denote by $\langle k\rangle$ the lattice of rank 1 generated by a vector $v$ with $(v, v)=k$. 
The following theorem describes the structure of unimodular indefinite lattices (see [99]).

Theorem 4.4. Let $M$ be a unimodular lattice of indefinite signature $(p, q)$. If $M$ is odd, then it is isometric to the lattice $I_{p, q}=\langle 1\rangle^{p} \perp\langle-1\rangle^{q}$. If $M$ is even, then $p-q \equiv 0 \bmod 8$ and $M$ or $M(-1)$ is isometric to the lattice $I I_{p, q}, p<q$, equal to the orthogonal sum $U^{p} \perp E_{8}^{\frac{q-p}{8}}$.

4.3. Reflection group of a lattice. Recall that the orthogonal group of a nondegenerate symmetric bilinear form on a finite-dimensional vector space over a field of characteristic $\neq 2$ is always generated by reflections. This does not apply to orthogonal groups of lattices.

Let $M$ be a nondegenerate quadratic lattice. A root vector in $M$ is a primitive vector $\alpha$ with $(\alpha, \alpha) \neq 0$ satisfying (4.1). A root vector $\alpha$ defines a reflection

$$
r_{\alpha}: x \mapsto x-\frac{2(\alpha, x)}{(\alpha, \alpha)} \alpha
$$

in $V=M_{\mathbb{R}}$ which leaves $M$ invariant. Obviously any vector $\alpha$ with $(\alpha, \alpha)= \pm 1$ or \pm 2 is a root vector. Suppose $(\alpha, \alpha)=2 k$. The linear function $M \rightarrow \mathbb{Z}, x \mapsto \frac{2(\alpha, x)}{(\alpha, \alpha)}$ defines a nontrivial element from $M^{*} / M$ of order $k$. Thus $k$ must divide the order of the discriminant group. In particular, all root vectors of a unimodular even lattice satisfy $(\alpha, \alpha)= \pm 2$.

We will be interested in positive definite lattices or hyperbolic lattices $M$ of signature $(n, 1)$. For such a lattice we define the reflection group $\operatorname{Ref}(M)$ of $M$ as the subgroup of $\mathrm{O}(M)$ generated by reflections $r_{\alpha}$, where $\alpha$ is a root vector with $(\alpha, \alpha)>0$. We denote by $\operatorname{Ref}_{k}(M)$ its subgroup generated by reflections in root vectors with $k=(\alpha, \alpha)$ (the $k$-reflection subgroup). We set

$$
\operatorname{Ref}_{-k}(M(-1))=\operatorname{Ref}_{k}(M) \text {. }
$$

Each group $\operatorname{Ref}_{k}(M)$ is a reflection group in corresponding hyperbolic or spherical space.

Suppose $M$ is of signature $(n, 1)$. Let

$$
\mathrm{O}(M)^{+}=\mathrm{O}(M) \cap \mathrm{O}(n, 1)^{+}, \quad \mathrm{O}(M)=\mathrm{O}(M)^{+} \times\{ \pm 1\},
$$

where $\mathrm{O}(n, 1)^{+}$is the subgroup of index 2 of $\mathrm{O}(n, 1)$ defined in section 2.2. Note that every $\operatorname{Ref}_{k}(M)$ is a normal subgroup of $\mathrm{O}(M)^{+}$.

Let $P$ be a fundamental polyhedron of $\operatorname{Ref}_{k}(M)$ in $H^{n}$. Since $\mathrm{O}(M)$ leaves invariant the set of root vectors $\alpha$ with fixed $(\alpha, \alpha)$, it leaves invariant the set of reflection hyperplanes of $\operatorname{Ref}_{k}(M)$. Hence, for any $g \in \mathrm{O}(M)^{+}$, there exists $s \in \operatorname{Ref}_{k}(M)$ such that $g(P)=s(P)$. This shows that

$$
\mathrm{O}(M)^{+}=\operatorname{Ref}_{k}(M) \rtimes S(P),
$$

where $S(P)$ is the subgroup of $\mathrm{O}(M)$ which leaves $P$ invariant.

Example 4.5. Let $M=E_{p, q, r}$ with finite $\operatorname{Ref}(M)$. Then $\operatorname{Ref}(M)=W(p, q, r)$ from Example 2.7, where $(p, q, r)=(1,1, n)\left(A_{n}\right),(2,2, n-2)\left(D_{n}\right),(2,3,3)\left(E_{6}\right),(2,3,4)$ $\left(E_{7}\right),(2,3,5)\left(E_{8}\right)$. We have $S(P)=\mathbb{Z} / 2 \mathbb{Z}\left(A_{n}, E_{6}, D_{n}, n \geq 5\right), S(P)=S_{3}\left(D_{4}\right)$ and $S(P)$ is trivial for $E_{7}, E_{8}$.

The standard notations for the finite reflection groups $W(p, q, r)$ are $W(T)$, where $T=A_{n}, D_{n}, E_{6}, E_{7}, E_{8}$. The corresponding lattices $E_{p, q, r}$ are called finite root 
lattices. Their reflection groups are the Weyl groups of the corresponding root systems.

In general $\operatorname{Ref}\left(E_{p, q, r}\right)$ is larger than the group $W(p, q, r)$ (see Example 4.11).

Example 4.6. Let $M=I I_{25,1}$ be an even unimodular hyperbolic lattice of rank 26. According to Theorem 4.4

$$
I I_{25,1} \cong U \perp E_{8}^{3} .
$$

The lattice $I I_{25,1}$ contains as a direct summand an even positive definite unimodular lattice $\Lambda$ of rank 24 with $(v, v) \neq 2$ for all $v \in \Lambda$. A lattice with such properties (which determine uniquely the isomorphism class) is called a Leech lattice. Thus $I I_{25,1}$ can also be described as

$$
I I_{25,1}=U \perp \Lambda .
$$

The description of $\operatorname{Ref}\left(I I_{25,1}\right)=\operatorname{Ref}_{2}\left(I I_{25,1}\right)$ was given by J. Conway [22]. The group admits a fundamental polytope $P$ whose reflection hyperplanes are orthogonal to the Leech roots, i.e. root vectors of the form $\left(f-\left(1+\frac{(v, v)}{2}\right) g, v\right)$, where $v \in \Lambda$ and $f, g$ is a basis of $U$ with Gram matrix $\left(\begin{array}{ll}0 & 1 \\ 1 & 0\end{array}\right)$. In other words, a choice of a decomposition (4.6) defines a fundamental polyhedron for the reflection group with fundamental roots equal to the Leech vectors. We have

$$
\mathrm{O}\left(I I_{25,1}\right)^{+}=\operatorname{Ref}\left(I I_{25,1}\right) \rtimes S(P),
$$

where $S(P) \cong \Lambda \rtimes \mathrm{O}(\Lambda)$.

Define a hyperbolic lattice $M$ to be reflective if its root vectors span $M$ and $\operatorname{Ref}(M)$ is of finite covolume (equivalently, its index in $\mathrm{O}(M)$ is finite). ${ }^{6}$ In the hyperbolic case the first condition follows from the second one. It is clear that the reflectivity property of $M$ is preserved when we scale $M$, i.e. replace $M$ with $M(k)$ for any positive integer $k$. The following nice result is due to F. Esselmann [42].

Theorem 4.7. Reflective lattices of signature $(n, 1)$ exist only if $n \leq 19$ or $n=21$.

The first example of a reflective lattice of rank 22 was given by Borcherds [12]. We will discuss this lattice later.

An important tool in the classification (yet unknown) of reflective lattices is the following lemma of Vinberg [112].

Lemma 4.8. Let $M$ be a hyperbolic reflective lattice. For any isotropic vector $v \in M$ the lattice $v^{\perp} / \mathbb{Z} v$ is a definite reflective lattice.

Another useful result is the following (see [18]).

Theorem 4.9. Suppose a reflective hyperbolic lattice $M$ decomposes as an orthogonal sum of a lattice $M^{\prime}$ and a definite lattice $K$. Then $M^{\prime}$ is reflective.

Examples 4.10. 1) A lattice of rank 1 is always reflective.

2) The lattice $U$ is reflective. The group $\mathrm{O}(U)$ is finite.

3) All finite root lattices and their orthogonal sums are reflective.

4) An odd lattice $I_{n, 1}=\langle 1\rangle^{n} \perp\langle-1\rangle$ is reflective if and only if $n \leq 20$. The Coxeter diagrams of their reflection groups can be found in [120], Chapter $6, \S 2$ $(n \leq 18)$ and in [119] $(n=18,19)$. Some of these diagrams are also discussed in

\footnotetext{
${ }^{6}$ This definition is closely related but differs from the definition of reflective hyperbolic lattices used in the works of V. Gritsenko and V. Nikulin on Lorentzian Kac-Moody algebras [52].
} 
[23], Chapter 28. The lattices $I_{n, 1}(2)$ are reflective for $n \leq 19$ but 2-reflective only for $n \leq 9$. For example, Figure 8 is the Coxeter diagram of the reflection group of the lattice $I_{16,1}$.

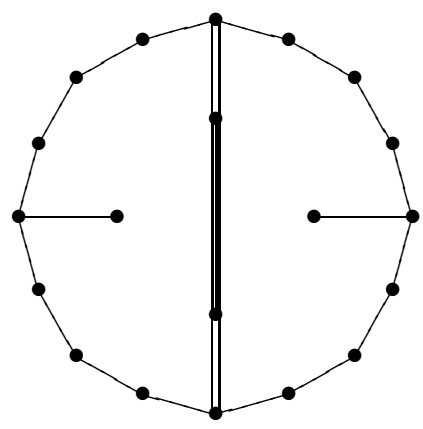

Figure 8

It is easy to see that the reflectivity property implies that all vertices not connected by the thick line correspond to roots $\alpha$ with $(\alpha, \alpha)=2$ and the remaining two vertices correspond to roots with $(\alpha, \alpha)=1$.

Many other examples of Coxeter diagrams for 2-reflective lattices can be found in [84].

5) Many examples (almost a classification) of reflective lattices of ranks 3 and 4 can be found in [97], [103].

All even hyperbolic lattices of rank $r>2$ for which $\operatorname{Ref}_{2}(M)$ is of finite covolume (2-reflective lattices) were found by V. Nikulin [84] $(r \neq 4)$ and E. Vinberg (unpublished) $(r=4)$ (a survey of Nikulin's results can be found in [29]). They exist only in dimension $\leq 19$.

Example 4.11. A hyperbolic lattice $E_{2,3, r}$ is 2-reflective if and only if $7 \leq r \leq 10$. A hyperbolic lattice $E_{2,4, r}$ is 2-reflective if and only if $r=5,6,7$. A hyperbolic lattice $E_{3,3, r}$ is 2-reflective if and only if $r=4,5,6$. This easily follows from Proposition 2.4. The reflection groups of the lattices $E_{2,3,7}, E_{3,3,4}$ and $E_{2,4,5}$ are quasi-Lanner and coincide with the Coxeter groups $W(2,3,7), W(3,3,4), W(2,4,5)$. The reflection groups of other lattices are larger than the corresponding groups $W(p, q, r)$. For example, the Coxeter diagram of $\operatorname{Ref}_{2}\left(E_{2,3,8}\right)=\operatorname{Ref}\left(E_{2,3,8}\right)$ is the one in Figure 9.

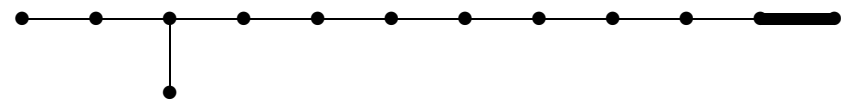

FiguRE 9

To prove this fact one first observes, using Proposition 2.4, that the Coxeter diagram defines a group of cofinite volume. The root vector corresponding to the extreme right vertex is equal to the vector $r+e$, where $r$ generates the kernel of the lattice $E_{2,3,6}$ embedded naturally in $E_{2,3,8}$ and the vector $e$ is the root vector corresponding to the extreme right vector in the subdiagram defining the sublattice isomorphic to $E_{2,3,7}$. 


\section{Automorphisms of Algebraic surfaces}

5.1. Quadratic lattices associated to an algebraic surface. Let $X$ be a complex projective algebraic surface. It has the underlying structure of a compact smooth oriented 4-manifold. Thus the cohomology group $H^{2}(X, \mathbb{Z})$ is a finitely generated abelian group equipped with a symmetric bilinear form

$$
H^{2}(X, \mathbb{Z}) \times H^{2}(X, \mathbb{Z}) \rightarrow \mathbb{Z}
$$

defined by the cup-product.

When we divide $H^{2}(X, \mathbb{Z})$ by the torsion subgroup we obtain a unimodular quadratic lattice $H_{X}$. We will denote the value of the bilinear form on $H_{X}$ induced by the cup product by $x \cdot y$ and write $x^{2}$ if $x=y$.

To compute its signature one uses the Hodge decomposition (depending on the complex structure of $X$ )

$$
H^{2}(X, \mathbb{C})=H^{2,0}(X) \oplus H^{1,1}(X, \mathbb{C}) \oplus H^{0,2}(X),
$$

where $\operatorname{dim} H^{2,0}=\operatorname{dim} H^{0,2}$ and is equal to the dimension $p_{g}(X)$ of the space of holomorphic differential 2 -forms on $X$. It is known that under the complex conjugation on $H^{2}(X, \mathbb{C})$ the space $H^{1,1}(X)$ is invariant and the space $H^{2,0}(X)$ is mapped to $H^{0,2}(X)$, and vice versa. One can also compute the restriction of the cup-product on $H^{2}(X, \mathbb{C})$ to each $H^{p, q}$ to conclude that the signature of the cup-product on $H^{2}(X, \mathbb{R})$ is equal to $\left(b_{2}^{+}, b_{2}^{-}\right)$, where $b_{2}^{+}=2 p_{g}+1$.

The parity of the lattice $H_{X}$ depends on the property of its first Chern class $c_{1}(X) \in H^{2}(X, \mathbb{Z})$. If is divisible by 2 in $H^{2}(X, \mathbb{Z})$, then $H_{X}$ is an even lattice.

The lattice $H_{X}$ contains an important primitive sublattice which depends on the complex structure of $X$. For any complex irreducible curve $C$ on $X$ its fundamental class $[C]$ defines a cohomology class in $H^{2}(X, \mathbb{Z})$. The $\mathbb{Z}$-span of these classes defines a subgroup of $H^{2}(X, \mathbb{Z})$, and its image $S_{X}$ in $H_{X}$ is called the Neron-Severi lattice (or the Picard lattice) of $X$. It is a sublattice of $H_{X}$ of signature $(1, \rho-1)$, where $\rho=\operatorname{rank} S_{X}$. An example of an element of positive norm is the class of a hyperplane section of $X$ in any projective embedding of $X$. Thus the lattice $S_{X}$ is hyperbolic in the sense of previous sections, and we can apply the theory of reflection groups to $S_{X}$.

It is known that the image in $H_{X}$ of the first Chern class $c_{1}(X) \in H^{2}(X, \mathbb{Z})$ belongs to the Picard lattice. The negative $K_{X}=-c_{1}(X)$ is the canonical class of $X$. It is equal to the image in $H^{2}(X, \mathbb{Z})$ of a divisor of zeros and poles of a holomorphic differential 2-form on $X$. We denote the image of $K_{X}$ in $H_{X}$ by $k_{X}$. For any $x \in S_{X}$ we define

$$
p_{a}(x)=x^{2}+x \cdot k_{X} .
$$

It is always an even integer. If $x$ is the image in $H_{X}$ of the fundamental class of a nonsingular complex curve $C$ on $X$, then $p_{a}(x)$ is equal to $2 g-2$, where $g$ is the genus of the Riemann surface $C$ (the adjunction formula). If $C$ is an irreducible complex curve with finitely many singular points, then this number is equal to $2 g_{0}-2+2 \delta$, where $g_{0}$ is the genus of a nonsingular model of $C$ (e.g. the normalization) and $\delta$ depends on the nature of singular points of $C$ (e.g. equal to their number if all singular points are ordinary nodes or cusps). This implies that $p_{a}(x)$ is always even. In particular, the sublattice

$$
S_{X}^{0}=k_{X}^{\perp}=\left\{x \in S_{X}: x \cdot k_{X}=0\right\}
$$


is always even. Its signature is equal to

$$
\operatorname{sign}\left(S_{X}^{0}\right)= \begin{cases}(1, \rho-1) & \text { if } k_{X}=0 \\ (0, \rho-2,1) & \text { if } k_{X}^{2}=0, k_{X} \neq 0 \\ (0, \rho-1) & \text { if } k_{X}^{2}>0 \\ (1, \rho-2) & \text { if } k_{X}^{2}<0\end{cases}
$$

A (-1)-curve (resp. (-2)-curve) on $X$ is a nonsingular irreducible curve $C$ of genus 0 (thus isomorphic to $\mathbb{P}^{1}(\mathbb{C})$ ) with $[C]^{2}=-1$ (resp. -2). By the adjunction formula this is equivalent to $C \cong \mathbb{P}^{1}(\mathbb{C})$ and $[C] \cdot K_{X}=-1$ (resp. 0$)$. A $(-1)$-curve appears as a fibre of a blow-up map $f: X \rightarrow Y$ of a point on a nonsingular algebraic surface $Y$. A (-2)-curve appears as a fibre of a resolution of an ordinary double point on a complex surface $Y$.

A surface $X$ which does not contain (-1)-curves is called minimal. The following results follow from the Enriques-Kodaira classification of complex algebraic surfaces (see $[9]$ ).

Theorem 5.1. Let $X$ be a minimal complex algebraic surface. Then one of the following cases occurs.

(1) $X \cong \mathbb{P}^{2}$ or there exists a regular map $f: X \rightarrow B$ to some nonsingular curve $B$ whose fibres are isomorphic to $\mathbb{P}^{1}$. Moreover $S_{X}=H_{X}$, and we are in precisely one of the following cases:

(i) $X \cong \mathbb{P}^{2}, S_{X} \cong\langle 1\rangle$, and $k_{X}=3 a$, where a is a generator.

(ii) $S_{X} \cong U$, and $k_{X}=2 a+2 b$, where $a, b$ are generators of $S_{X}$ with the Gram matrix $\left(\begin{array}{ll}0 & 1 \\ 1 & 0\end{array}\right)$.

(iii) $S_{X} \cong I_{1,1}=\langle 1\rangle \perp\langle-1\rangle$ and $k_{X}=2 a+2 b$, where $a, b$ are generators of $S_{X}$ with the Gram matrix $\left(\begin{array}{cc}1 & 0 \\ 0 & -1\end{array}\right)$.

(2) $k_{X}=0$ :

(i) $H_{X} \cong U^{3} \perp E_{8}(-1)^{2}, S_{X}$ is an even lattice of signature $(1, \rho-1)$, where $1 \leq \rho \leq 20$;

(ii) $H_{X}=S_{X} \cong U \perp E_{8}(-1)$;

(iii) $H_{X} \cong U^{3}, S_{X}$ is an even lattice of signature $(1, \rho-1)$, where $1 \leq \rho \leq$ 4

(iv) $H_{X}=S_{X} \cong U$.

(3) $k_{X} \neq 0, k_{X}^{2}=0, S^{0} / \mathbb{Z} k_{X}$ is a negative definite lattice.

(4) $k_{X}^{2}>0, K_{X} \cdot[C] \geq 0$ for any curve $C$ on $X, S_{X}^{0}$ is a negative definite lattice.

The four cases (1)-(4) correspond to the four possible values of the Kodaira dimension $\kappa(X)$ of $X$ equal to $-\infty, 0,1,2$, respectively. Recall that $\kappa(X)$ is equal to the maximal possible dimension of the image of $X$ under a rational map given by some multiple of the canonical linear system on $X$. The four subcases (i)-(iv) in (2) correspond to K3-surfaces, Enriques surfaces, abelian surfaces (complex algebraic tori), and hyperelliptic surfaces, respectively.

Let $\operatorname{Aut}(X)$ denote the group of automorphisms of $X$ (as an algebraic variety or as a complex manifold). Any $g \in \operatorname{Aut}(X)$ acts naturally on $H_{X}$ via the pull-backs of cohomology classes. Since the latter is compatible with the cup-product, the action preserves the structure of a quadratic lattice on $H_{X}$ and also leaves invariant the sublattice $S_{X}$. This defines a homomorphism

$$
\operatorname{Aut}(X) \rightarrow \mathrm{O}\left(S_{X}\right), g \mapsto\left(g^{*}\right)^{-1} .
$$


Since $g^{*}\left(K_{X}\right)=K_{X}$, we see that the image of this homomorphism is contained in the stabilizer subgroup $\mathrm{O}\left(S_{X}\right)_{k_{X}}$ of the vector $k_{X}$. In particular, it induces a homomorphism

$$
a: \operatorname{Aut}(X) \rightarrow \mathrm{O}\left(S_{X}^{0}\right) .
$$

The group $\operatorname{Aut}(X)$ is a topological group whose connected component of the identity $\operatorname{Aut}(X)^{0}$ is a complex Lie group. One can show that $\operatorname{Aut}(X)^{0}$ acts identically on $S_{X}$ and the kernel of the induced map of the quotient group $\operatorname{Aut}(X) / \operatorname{Aut}(X)^{0}$ is finite (see [31]). The group Aut $(X)^{0}$ can be nontrivial only for surfaces of Kodaira dimension $-\infty$, or abelian surfaces, or surfaces of Kodaira dimension 1 isomorphic to some finite quotients of the products of two curves, one of which is of genus 1 . It follows from Theorem 5.1 that $\operatorname{Aut}(X)$ is always finite for surfaces of Kodaira dimension 2 .

5.2. Rational surfaces. A rational surface $X$ is a nonsingular projective algebraic surface birationally isomorphic to $\mathbb{P}^{2}$. We will be interested only in basic rational surfaces, i.e. algebraic surfaces admitting a regular birational map $\pi: X \rightarrow \mathbb{P}^{2}{ }^{7}$

It is known that any birational regular map of algebraic surfaces is equal to the composition of blow-ups of points ([55]). Applying this to the map $\pi$, we obtain a factorization

$$
\pi: X=X_{N} \stackrel{\pi_{N}}{\longrightarrow} X_{N-1} \stackrel{\pi_{N-1}}{\longrightarrow} \ldots \stackrel{\pi_{2}}{\longrightarrow} X_{1} \stackrel{\pi_{1}}{\longrightarrow} X_{0}=\mathbb{P}^{2},
$$

where $\pi_{i}: X_{i} \rightarrow X_{i-1}$ is the blow-up of a point $x_{i} \in X_{i-1}$. Let

$$
E_{i}=\pi_{i}^{-1}\left(x_{i}\right), \quad \mathcal{E}_{i}=\left(\pi_{i+1} \circ \ldots \pi_{N}\right)^{-1}\left(E_{i}\right) .
$$

Let $e_{i}$ denote the cohomology class $\left[\mathcal{E}_{i}\right]$ of the (possibly reducible) curve $\mathcal{E}_{i}$. It satisfies $e_{i}^{2}=e_{i} \cdot k_{X}=-1$. One easily checks that $e_{i} \cdot e_{j}=0$ if $i \neq j$. Let $e_{0}=\pi^{*}([\ell])$, where $\ell$ is a line in $\mathbb{P}^{2}$. We have $e_{0} \cdot e_{i}=0$ for all $i$. The classes $e_{0}, e_{1}, \ldots, e_{N}$ form a basis in $S_{X}=H_{X}$ which we call a geometric basis. The Gram matrix of a geometric basis is the diagonal matrix $\operatorname{diag}[1,-1, \ldots,-1]$. Thus the factorization (5.4) defines an isomorphism of quadratic lattices

$$
\phi_{\pi}: I_{1, N} \rightarrow S_{X}, \mathbf{e}_{i} \mapsto e_{i},
$$

where $\mathbf{e}_{0}, \ldots, \mathbf{e}_{N}$ is the standard basis of $I_{1, N}$. It follows from the formula for the behavior of the canonical class under a blow-up that $k_{X}$ is equal to the image of the vector

$$
k_{N}=-3 \mathbf{e}_{0}+\mathbf{e}_{1}+\ldots+\mathbf{e}_{N} .
$$

This implies that the lattice $S_{X}^{0}$ is isomorphic to the orthogonal complement $k_{N}^{\perp}$ in $I_{1, N}$.

For $N \geq 3$, the vectors

$$
\mathbf{a}_{1}=\mathbf{e}_{0}-\mathbf{e}_{1}-\mathbf{e}_{2}-\mathbf{e}_{3}, \quad \mathbf{a}_{2}=\mathbf{e}_{1}-\mathbf{e}_{2}, \quad \ldots \quad, \quad \mathbf{a}_{N}=\mathbf{e}_{N-1}-\mathbf{e}_{N}
$$

form a basis of $k_{N}^{\perp}$ with Gram matrix equal to $-2 C$, where $C$ is the Gram matrix of the Coxeter group $W\left(E_{N}\right):=W(2,3, N-3)$ if $N \geq 4$ and $W\left(E_{3}\right)=W\left(A_{2} \times A_{1}\right)$

\footnotetext{
${ }^{7}$ For experts: Nonbasic rational surfaces are easy to describe: they are either minimal rational surfaces different from $\mathbb{P}^{2}$ or surfaces obtained from minimal ruled surfaces $\mathbf{F}_{n}, n \geq 2$, by blowing up points on the exceptional section and their infinitely near points. The automorphism groups of nonbasic rational surfaces are easy to describe, and they are rather dull.
} 
if $N=3$. This embeds the lattice

$$
E_{N}= \begin{cases}A_{2} \perp A_{1} & \text { if } N=3, \\ E_{2,3, N-3} & \text { if } N \geq 4\end{cases}
$$

in the lattice $I_{N, 1}$ with orthogonal complement generated by $k_{N}$. The restriction of $\phi_{\pi}$ to $k_{N}^{\perp}$ defines an isomorphism of lattices

$$
\phi_{\pi}: E_{N}(-1) \rightarrow S_{X}^{0}
$$

Let us identify the Coxeter group $W\left(E_{N}\right)$ with the subgroup of $\mathrm{O}\left(I_{1, N}\right)$ generated by the reflections in the vectors $\mathbf{a}_{i}$ from (5.6). A choice of a geometric basis in $S_{X}$ defines an isomorphism from $W\left(E_{N}\right)$ to a subgroup of $\mathrm{O}\left(S_{X}^{0}\right)$ generated by reflections in vectors $\alpha_{i}=\phi_{\pi}\left(\mathbf{a}_{i}\right)$. It is contained in the reflection group $\operatorname{Ref}_{-2}\left(S_{X}^{0}\right)$.

Theorem 5.2. The image $W_{X}$ of $W\left(E_{N}\right)$ in $\mathrm{O}\left(S_{X}^{0}\right)$ does not depend on the choice of a geometric basis. The image of the homomorphism a : Aut $(X) \rightarrow \mathrm{O}\left(S_{X}^{0}\right)$ is contained in $W_{X}$.

Proof. To prove the first assertion it suffices to show that the transition matrix of two geometric bases defines an orthogonal transformation of $I_{1, N}$ which is the product of reflections in vectors $\mathbf{a}_{i}$. Let $\left(e_{0}, \ldots, e_{N}\right)$ and $\left(e_{0}^{\prime}, \ldots, e_{N}^{\prime}\right)$ be two geometric bases and

$$
e_{0}^{\prime}=m_{0} e_{0}-m_{1} e_{1}-\ldots-m_{N} e_{N}
$$

For any curve $C$ on $X$, we have $e_{0}^{\prime} \cdot[C]=\pi^{*}([\ell]) \cdot[C]=[\ell] \cdot\left[\pi^{\prime}(C)\right] \geq 0$. Intersecting $e_{0}^{\prime}$ with $e_{i}$ we obtain that $m_{0}>0, m_{i} \geq 0, i>0$. We have

$$
\begin{aligned}
1 & =e_{0}^{2}=m_{0}^{2}-m_{1}^{2}-\ldots-m_{N}^{2}, \\
-3 & =e_{0} \cdot k_{X}=-3 m_{0}+m_{1}+\ldots+m_{N} .
\end{aligned}
$$

Applying the reflections in vectors $\alpha_{i}=\phi_{\pi}\left(\mathbf{a}_{i}\right), i>1$, we may assume that $m_{1} \geq$ $m_{2} \geq \ldots \geq m_{N}$. Now we use the following inequality (Noether's inequality):

$$
m_{0}>m_{1}+m_{2}+m_{3} \text { if } m_{0}>1 \text {. }
$$

To see this we multiply the second equality in (5.8) by $m_{3}$ and then subtract from the first one to get

$$
m_{1}\left(m_{1}-m_{3}\right)+m_{2}\left(m_{2}-m_{3}\right)-\sum_{i \geq 4} m_{i}\left(m_{3}-m_{i}\right)=m_{0}^{2}-1-3 m_{3}\left(m_{0}-1\right) .
$$

This gives

$$
\begin{aligned}
\left(m_{0}-1\right)\left(m_{1}\right. & \left.+m_{2}+m_{3}-m_{0}-1\right)=\left(m_{1}-m_{3}\right)\left(m_{0}-1-m_{1}\right) \\
& +\left(m_{2}-m_{3}\right)\left(m_{0}-1-m_{2}\right)+\sum_{i \geq 4} m_{i}\left(m_{3}-m_{i}\right) .
\end{aligned}
$$

The first inequality in (5.8) implies that $m_{0}^{2}-m_{1}^{2}>0$; hence $m_{0}-m_{i} \geq 1$. Thus the right-hand side is nonnegative, so the left-hand side is too. This proves the claim. Now consider the reflection $s=r_{\alpha_{1}}$. Applying it to $e_{0}^{\prime}$ we get

$$
\begin{aligned}
s\left(e_{0}^{\prime}\right)= & e_{0}^{\prime}+\left(\left(e_{0}-e_{1}-e_{2}-e_{3}\right) \cdot e_{0}^{\prime}\right)\left(e_{0}-e_{1}-e_{2}-e_{3}\right) \\
= & \left(2 m_{0}-m_{1}-m_{2}-m_{3}\right) e_{0}-\left(m_{0}-m_{2}-m_{3}\right) e_{1} \\
& \quad-\left(m_{0}-m_{1}-m_{3}\right) e_{2}-\left(m_{0}-m_{1}-m_{2}\right) e_{3} .
\end{aligned}
$$

Using (5.9), we obtain that the matrix $S \cdot A$, where $S$ is the matrix of $s$, has the first column equal to $\left(m_{0}^{\prime},-m_{1},-\ldots,-m_{N}^{\prime}\right)$ with $m_{0}^{\prime}<m_{0}$ and $m_{i}^{\prime} \geq 0$ for $i>0$. 
Since our transformations are isometries of $S_{X}$, the inequalities (5.8) hold for the vector $\left(m_{0}^{\prime},-m_{1},-\ldots,-m_{N}^{\prime}\right)$. So, we repeat the argument in order to decrease $m_{0}^{\prime}$. After finitely many steps we get the transformation with first column vector equal to $(1,0, \ldots, 0)$. Now the matrix being the orthogonal matrix of the quadratic form $x_{0}^{2}-x_{1}^{2}-\ldots-x_{N}^{2}$ must have the first row equal to $(1,0, \ldots, 0)$. Thus the remaining rows and columns define an orthogonal matrix of the quadratic form $x_{1}^{2}+\ldots+x_{N}^{2}$ with integer entries. This implies that after reordering the columns and the rows we get a matrix with \pm 1 at the diagonal and zero elsewhere. It remains to use the fact that the transformation leaves the vector $k_{N}$ invariant to conclude that the matrix is the identity. Thus the transition matrix is the product of the matrices corresponding to reflections in vectors $\alpha_{i}, i=1, \ldots, N$.

Let us prove the last statement. Suppose $g^{*}$ is the identity on $S_{X}$. Then $g^{*}\left(e_{N}\right)=\left[g^{-1}\left(\mathcal{E}_{N}\right)\right]=e_{N}$. Since $\mathcal{E}_{N}^{2}<0$, it is easy to see that $\mathcal{E}_{N}$ is homologous to $g^{-1}\left(\mathcal{E}_{N}\right)$ only if $g\left(\mathcal{E}_{N}\right)=\mathcal{E}_{N}$. This implies that $g$ descends to the surface $X_{N-1}$. Replacing $X$ with $X_{N-1}$ and repeating the argument, we see that $g$ descends to $X_{N-2}$. Continuing in this way we obtain that $g^{*}$ is the identity and descends to a projective automorphism $g^{\prime}$ of $\mathbb{P}^{2}$. If all curves $\mathcal{E}_{i}$ are irreducible, their images on $\mathbb{P}^{2}$ form an ordered set of $N$ distinct points which must be preserved under $g^{\prime}$. Since a square matrix of size $3 \times 3$ has at most 3 linear independent eigenvectors, we see that $g^{\prime}$, and hence $g$, must be the identity. The general case requires a few more techniques to prove, and we omit the proof.

The main problem is to describe all possible subgroups of the Weyl group $W\left(E_{N}\right)$ which can be realized as the image of a group $G$ of automorphisms of a rational surface obtained by blowing-up $N$ points in the plane.

First of all we may restrict ourselves to minimal pairs $(X, G \subset \operatorname{Aut}(X))$. Minimal means that any $G$-equivariant birational regular map $f: X \rightarrow X^{\prime}$ of rational surfaces must be an isomorphism. A factorization (5.4) of $\pi^{\prime}: X^{\prime} \rightarrow \mathbb{P}^{2}$ can be extended to a factorization $\pi: X \rightarrow \mathbb{P}^{2}$ in such a way that the geometric basis $\phi_{\pi^{\prime}}(\mathbf{a})$ of $S_{X^{\prime}}$ can be extended to a geometric basis $\phi_{\pi}(\mathbf{a})$ of $S_{X}$. This gives a natural inclusion $W_{X^{\prime}} \subset W_{X}$ such that the image of $G$ in $W_{X^{\prime}}$ coincides with the image of $G$ in $W_{X}$.

The next result goes back to the classical work of S. Kantor [63] and now easily follows from an equivariant version of Mori's theory of minimal models.

Theorem 5.3. Let $G$ be a finite group of automorphisms of a rational surface $X$ making a minimal pair $(X, G)$. Then either $X \cong \mathbb{P}^{2}$, or $X$ is a conic bundle with $\left(S_{X}\right)^{G} \cong \mathbb{Z}^{2}$ or $\mathbb{Z}$, or $X$ is a Del Pezzo surface with $\left(S_{X}\right)^{G} \cong \mathbb{Z}$.

Here a Del Pezzo surface is a rational surface $X$ with ample $-K_{X} \cdot{ }^{8}$ Each Del Pezzo surface is isomorphic to either $\mathbb{P}^{2}$ or $\mathbb{P}^{1} \times \mathbb{P}^{1}$, or it admits a factorization (5.4) with $N \leq 8$ and the images of the points $x_{1}, \ldots, x_{N}$ in $\mathbb{P}^{2}$ are all distinct and satisfy the following:

- no three are on a line,

- no six are on a conic,

- not all are contained on a plane cubic with one of them being its singular point $(N=8)$.

\footnotetext{
${ }^{8}$ This means that in some projective embedding of $X$ its positive multiple is equal to the fundamental class of a hyperplane section.
} 
A conic bundle is a rational surface which admits a regular map to a nonsingular curve with fibres isomorphic to a conic (nonsingular or the union of two distinct lines).

A partial classification of finite groups $G$ which can be realized as groups of automorphisms of some rational surface was given by S. Kantor (for a complete classification and the history of the problem see [33]).

Example 5.4. Let $X$ be a Del Pezzo surface with $N+1=\operatorname{rank} S_{X}$. We refer to the number $9-N$ as the degree of $X$. A Del Pezzo surface of degree $d>2$ is isomorphic to a nonsingular surface of degree $d$ in $\mathbb{P}^{d}$. The most famous example is a cubic surface in $\mathbb{P}^{3}$ with 27 lines on it. The Weyl group $W\left(E_{6}\right)$ is isomorphic to the group of 27 lines on a cubic surface, i.e. the subgroup of the permutation group $\Sigma_{27}$ which preserves the incidence relation between the lines. Although the group of automorphisms of a general cubic surface is trivial, some special cubic surfaces admit nontrivial finite automorphism groups. All of them were essentially classified in the 19th century.

The situation with infinite groups is more interesting and difficult. Since $E_{N}$ is negative definite for $N \leq 8$, a basic rational surface $X$ with infinite automorphism group is obtained by blowing up $N \geq 9$ points. It is known that when the points are in general position, in some precisely defined sense, the group $\operatorname{Aut}(X)$ is trivial [57], [67]. So surfaces with nontrivial automorphisms are obtained by blowing up a set of points in some special position.

Example 5.5. Let $X$ be obtained by blowing up 9 points $x_{1}, \ldots, x_{9}$ contained in two distinct irreducible plane cubic curves $F, G$. The surface admits a fibration $X \rightarrow \mathbb{P}^{1}$ with general fibre an elliptic curve. The image of each fibre in $\mathbb{P}^{2}$ is a plane cubic from the pencil of cubics spanned by the curves $F, G$. The exceptional curves $\mathcal{E}_{1}, \ldots, \mathcal{E}_{9}$ are sections of this fibration. Fix one of them, say $\mathcal{E}_{1}$, and equip each nonsingular fibre $X_{t}$ with the group law with the zero point $X_{t} \cap \mathcal{E}_{1}$. Take a point $x \in X_{t}$ and consider the sum $x+p_{i}(t)$, where $p_{i}(t)=X_{t} \cap \mathcal{E}_{i}$. This defines an automorphism on an open subset of $X$ which can be extended to an automorphism $g_{i}$ of $X$. When the points $x_{1}, \ldots, x_{9}$ are general enough, the automorphisms $g_{2}, \ldots, g_{9}$ generate a free abelian group of rank 8 . In general case it is a finitely generated group of rank $\leq 8$. In the representation of $\operatorname{Aut}(X)$ in $W_{X} \cong W\left(E_{9}\right)$ the image of this group is the lattice subgroup of the euclidean reflection group of type $E_{8}$.

This example can be generalized by taking general points $x_{1}, \ldots, x_{9}$ with the property that there exists an irreducible curve of degree $3 m$ such that each $x_{i}$ is its singular point of multiplicity $m$. In this case the image of $\operatorname{Aut}(X)$ in $W\left(E_{9}\right)$ is the subgroup of the lattice subgroup $\mathbb{Z}^{8}$ such that the quotient group is isomorphic to $(\mathbb{Z} / m \mathbb{Z})^{8}$ (see $\left.[36],[46]\right)$.

Example 5.6. Let $X$ be obtained by blowing up 10 points $x_{1}, \ldots, x_{10}$ with the property that there exists an irreducible curve of degree 6 with ordinary double points at each $x_{i}$ (a Coble surface). The image of $\operatorname{Aut}(X)$ in $W_{X} \cong W\left(E_{10}\right)$ is the subgroup

$$
W\left(E_{10}\right)(2)=\left\{g \in W\left(E_{10}\right): g(x)-x \in 2 E_{10} \text { for all } x \in E_{10}\right\}
$$

(see $[20])$. This group is the smallest normal subgroup which contains the involution of the lattice $E_{10}=U \perp E_{8}$ equal to $\left(\mathrm{id}_{U},-\mathrm{id}_{E_{8}}\right)$. 
Example 5.7. Let $(W, S)$ be a Coxeter system with finite set $S$. A Coxeter element of $(W, S)$ is the product of elements of $S$ taken in some order. Its conjugacy class does not depend on the order if the Coxeter diagram is a tree ([14], Chapter V, $\S 6$, Lemma 1). The order of a Coxeter element is finite if and only if $W$ is finite. Let $h_{N}$ be a Coxeter element of $W=W\left(E_{N}\right)$. In a recent paper [78] C. McMullen realizes $h_{N}$ by an automorphism of a rational surface. The corresponding surface $X$ is obtained by blowing up $N$ points in special position lying on a cuspidal plane cubic curve. One can check that for $N=9$ or 10 a Coxeter element does not belong to the subgroup described in the previous two examples. It is not known whether a rational surface realizing a Coxeter element is unique up to isomorphism for $N \geq 9$. It is known to be unique for $N \leq 8$. For example, for $N=6$ the surface is isomorphic to the cubic surface

$$
T_{0}^{3}+T_{1}^{3}+T_{3}^{2} T_{1}+T_{2}^{2} T_{3}=0 .
$$

The order of $h_{6}$ is equal to 12 .

Until very recently, all known examples of minimal pairs $(X, G)$ with infinite $G$ satisfied the following condition:

- There exists $m>0$ such that the linear system $\left|-m K_{X}\right|$ is not empty or, in another words, the cohomology class $m c_{1}(X)$ can be represented by an algebraic curve.

Without the minimality condition the necessity of this condition was conjectured by M. Gizatullin, but a counter-example was found by B. Harbourne [54]. A recent preprint of Eric Bedford and Kyounghee Kim [10] contains an example of a minimal surface with infinite automorphism group with $\left|-m K_{X}\right|=\emptyset$ for all $m>0$.

5.3. K3 surfaces. These are surfaces from case 2 (i) of Theorem 5.1. They are characterized by the conditions

$$
c_{1}(X)=0, \quad H^{1}(X, \mathbb{C})=0 .
$$

In fact, all K3 surfaces are simply connected and belong to the same diffeomorphism type.

Examples 5.8. 1) $X$ is a nonsingular surface of degree 4 in $\mathbb{P}^{3}$.

2) $X$ is the double cover of a rational surface $Y$ branched along a nonsingular curve $W$ whose cohomology class $[W]$ is equal to $-2 K_{X}$. For example, one may take $Y=\mathbb{P}^{2}$ and $W$ a nonsingular curve of degree 6 . Or, one takes $Y=\mathbb{P}^{1} \times \mathbb{P}^{1}$ and $W$ a curve of bi-degree $(4,4)$.

3) Let $A$ be a compact complex torus which happens to be a projective algebraic variety. This is a surface from case 2 (iii). The involution $\tau: a \mapsto-a$ has 16 fixed points, and the orbit space $A /(\tau)$ acquires 16 ordinary double points. A minimal nonsingular surface birationally equivalent to the quotient is a K3 surface, called the Kummer surface associated to $A$.

Let $\operatorname{Aut}(X)$ be the group of biregular automorphisms of $X$. It is known that $X$ does not admit nonzero holomorphic vector fields and hence the Lie algebra of the maximal Lie subgroup of $\operatorname{Aut}(X)$ is trivial. This shows that the $\operatorname{group} \operatorname{Aut}(X)$ is a discrete topological group and the kernel of the natural representation (5.2) of $\operatorname{Aut}(X)$ in $\mathrm{O}\left(S_{X}\right)$ is a finite group. 
Remark 5.9. One can say more about the kernel $H$ of homomorphism (5.2) (see [84], §10). Let $\chi: \operatorname{Aut}(X) \rightarrow \mathbb{C}^{*}$ be the one-dimensional representation of $\operatorname{Aut}(X)$ in the space $\Omega^{2}(X)$ of holomorphic 2 -forms on $X$. The image of $\chi$ is a cyclic group of some order $n$. First Nikulin proves that the value of the Euler function $\phi(n)$ divides $22-\operatorname{rank} S_{X}$. Next he proves that the restriction of $\chi$ to $H$ is injective. This implies that $H$ is a cyclic group of order dividing $n$. All possible values of $n$ which can occur are known (see [69]). The largest one is equal to 66 and can be realized for a K3 surface birationally isomorphic to a surface in the weighted projective space $\mathbb{P}(1,6,22,33)$ given by the equation $x^{66}+y^{11}+z^{3}+w^{2}=0$.

It follows from the adjunction formula that any smooth rational curve on a K3 surface is a $(-2)$-curve. The class of this curve in $S_{X}$ defines a reflection. Let $W_{X}^{+}$ denote the subgroup of $\mathrm{O}\left(S_{X}\right)$ generated by these reflections.

\section{Proposition 5.10.}

$$
\operatorname{Ref}_{-2}\left(S_{X}\right)=W_{X}^{+}
$$

Proof. Let $C$ be an irreducible curve on $X$. By the adjunction formula, $[C]^{2} \geq-2$ and $C^{2}=-2$ if and only if $C$ is a $(-2)$-curve. We call a divisor class effective if it can be represented by a (possibly reducible) algebraic curve on $X$. Using the Riemann-Roch Theorem, one shows that any divisor class $x$ with $x^{2} \geq-2$ is either effective or its negative is effective. Let $D=\sum_{i \in I} C_{i}$ be any algebraic curve written as a sum of its irreducible components. We have $[D] \cdot[C] \geq 0$ for any irreducible curve $C$ unless $C$ is an irreducible component of $D$ with $C^{2}=-2$. A divisor class $x$ is called nef if $x \cdot d \geq 0$ for any effective divisor class $d$. It is not difficult to show that the $W_{X}^{+}$-orbit of any effective divisor $x$ with $x^{2} \geq 0$ contains a unique nef divisor (use that $x \cdot e<0$ for some effective $e$ with $e^{2}=-2$ implying $\left.r_{e}(x) \cdot e>0\right)$. Let $V_{X}^{0}=\left\{x \in V_{X}: x^{2}>0\right\}$. We take for the model of the hyperbolic space $H^{n}$ associated with $S_{X}$ the connected component of $V_{X}^{0} / \mathbb{R}_{+} \subset \mathbb{P}\left(V_{X}\right)$ which contains the images of effective divisors from $V_{X}^{0}$. Then the image $P^{+}$in $H^{n}$ of the convex hull $N$ of nef effective divisors from $V_{X}^{0}$ is a fundamental polytope for the reflection group $W_{X}^{+}$. Its fundamental roots are the classes of $(-2)$-curves. Let $r_{\alpha}$ be a reflection from $\operatorname{Ref}_{-2}\left(S_{X}\right)$. Replacing $\alpha$ with $-\alpha$ we may assume that $\alpha$ is effective. Suppose $\alpha$ is a fundamental root for a fundamental polytope $P$ of $\operatorname{Ref}_{-2}\left(S_{X}\right)$ which is contained in $P^{+}$. Since all vectors from $N$ satisfy $x \cdot \alpha \geq 0$, we see that $P^{+} \subset P$ and hence $P=P^{+}$. This shows that $W_{X}^{+}$and $\operatorname{Ref}_{-2}\left(S_{X}\right)$ are defined by the same convex polytope and hence the groups are equal.

The following result follows from the fundamental Global Torelli Theorem for K3 surfaces due to I. Shafarevich and I. Pjatetsky-Shapiro [94].

Theorem 5.11. Let $A_{X}$ be the image of Aut $(X)$ in $\mathrm{O}\left(S_{X}\right)$. Let $G$ be the subgroup of $\mathrm{O}\left(S_{X}\right)$ generated by $W_{X}^{+}$and $A_{X}$. Then $G=W_{X}^{+} \rtimes A_{X}$, and its index in $\mathrm{O}\left(S_{X}\right)$ is finite.

The difficult part is the finiteness of the index.

Corollary 5.1. The following assertions are equivalent:

- $W_{X}^{+}$is of finite index in $\mathrm{O}\left(S_{X}\right)$;

- Aut $(X)$ is a finite group;

- the (-2)-reflection group of $S_{X}$ admits a fundamental polytope with finitely many faces defined by the classes of smooth rational curves; 
- $S_{X}(-1)$ is a 2-reflective lattice.

The third property implies that the set of $(-2)$-curves is finite if $\operatorname{Aut}(X)$ is finite, but the converse is not true.

Figures 10, 11, and 12 show the even 2-reflective lattices of rank 17, 18 and 19, the Coxeter diagrams of the reflection groups $\operatorname{Ref}_{-2}\left(S_{X}\right)$, and the corresponding K3 surfaces.
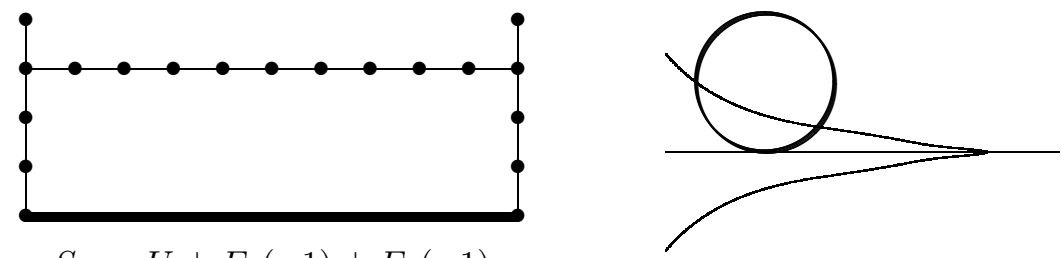

$$
S_{X}=U \perp E_{8}(-1) \perp E_{7}(-1)
$$

FigURE 10
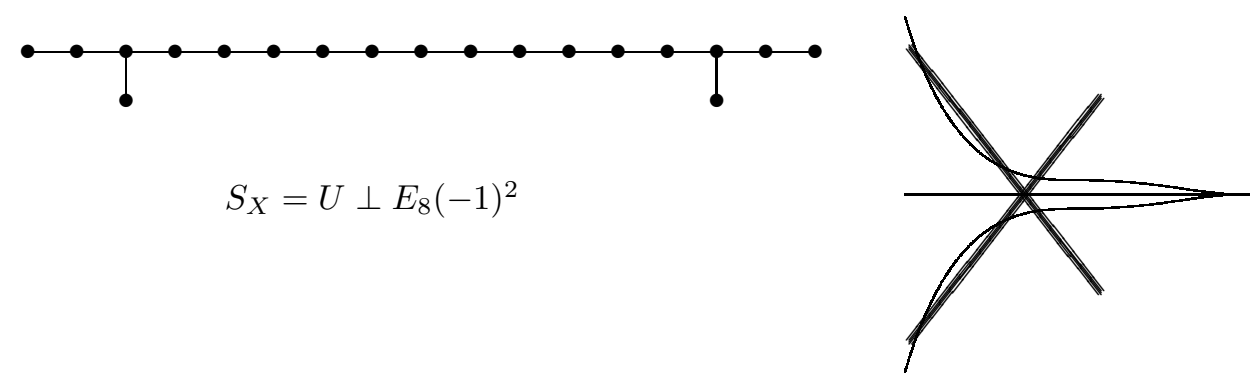

Figure 11
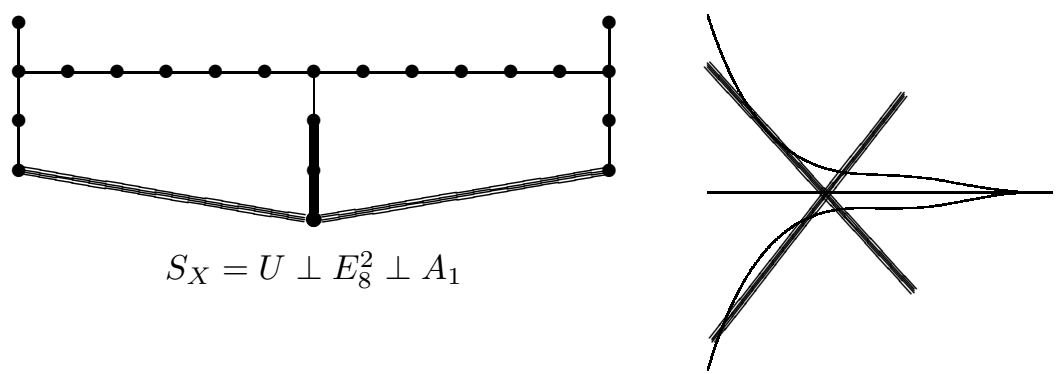

FIGURE 12

The K3 surface is birationally isomorphic to the surface obtained as the double cover of $\mathbb{P}^{2}$ branched along the curve of degree 2 drawn thick, followed by the double 
cover along the proper inverse transform of the remaining curve of degree 4 (the cuspidal cubic and its cuspidal tangent line).

It follows from the classification of 2-reflective lattices (see section 4) that each of them is isomorphic to the lattice $S_{X}$ for some K3-surface $X$.

A similar assertion for any even reflective lattice is not true for the following trivial reason. Replacing $M$ by $M(2 k)$ for some $k$, we obtain a reflective lattice with discriminant group whose minimal number of generators $s \geq \operatorname{rank} M$. Suppose $\operatorname{rank} M \geq 12$ and $M(k)$ is primitively embedded in $L_{K 3}$. Its orthogonal complement is a lattice of rank $22-12 \leq 10$ with isomorphic discriminant group generated by $\leq 10$ elements. This is a contradiction.

A more serious reason is the existence of an even reflective hyperbolic lattice $M$ of rank 22. One can take $M=U \perp D_{20}$ realized as the sublattice of $I_{19,1}$ of vectors with even $v^{2}$. The reflectivity of $M$ was proven by R. Borcherds [12]. Since rank $S_{X} \leq 20$ for any complex K3 surface $X$, the lattice $M$ cannot be isomorphic to $S_{X}$. However, the lattice $M$ is realized as the Picard lattice of a K3 surface over an algebraically closed field of characteristic 2 isomorphic to a quartic surface in $\mathbb{P}^{3}$ with equation

$$
T_{0}^{4}+T_{1}^{4}+T_{2}^{4}+T_{3}^{4}+T_{0}^{2} T_{1}^{2}+T_{0}^{2} T_{2}^{2}+T_{1}^{2} T_{2}^{2}+T_{0} T_{1} T_{2}\left(T_{0}+T_{1}+T_{2}\right)=0
$$

(see [35]). Note that over a field of positive characteristic the Hodge structure and the inequality $\rho=\operatorname{rank} S_{X} \leq 20$ does not hold. However, one can show that for any K3 surface over an algebraically closed field of positive characteristic

$$
\rho \leq 22, \quad \rho \neq 21,
$$

K3 surfaces with $\rho=22$ are called supersingular (in the sense of Shioda). Observe the striking analogy of inequalities (5.11) with the inequalities from Theorem 4.7.

Besides scaling, one can consider the following operations over nondegenerate quadratic lattices which preserve the reflectivity property (see [97]). The first operation replaces a lattice $M$ with $p^{-1}\left(M \cap p^{2} M^{*}\right)+M$ for any $p$ dividing the discriminant of $M$. This allows one to replace $M$ with a lattice such that the exponent of the discriminant groups is square free. The second operation replaces $M$ with $N(p)$, where $N=M^{*} \cap p^{-1} M$. This allows one to replace $M$ with a lattice such that the largest power $a$ of $p$ dividing the discriminant of $M$ satisfies $a \leq \frac{1}{2} \operatorname{rank} M$.

I conjecture that up to scaling and the above two operations any even hyperbolic reflective lattice is isomorphic to the lattice $S_{X}(-1)$ for some K3 surface defined over an algebraically closed field of characteristic $p \geq 0$.

It is known that the lattice $S_{X}(-1)$ for a supersingular K3-surface $X$ over a field of characteristic $p>0$ is always of rank 22 and its discriminant group is isomorphic to a $p$-elementary group $(\mathbb{Z} / p \mathbb{Z})^{2 \sigma}, \sigma \leq 10$ (see [96]). No two such lattices are equivalent in the sense of the operations on lattices described above. There is only one such reflective lattice, namely $U \perp D_{20}(-1)$.

There are only a few cases where one can compute explicitly the automorphism group of a K3 surface when it is infinite and the rank of $S_{X}$ is large. This requires one to construct explicitly a Coxeter polytope of $\operatorname{Ref}_{2}\left(S_{X}(-1)\right)$ which is of infinite volume. As far as I know this has been accomplished only in the following cases:

- $X$ is the Kummer surface of the Jacobian variety of a general curve of genus $2([70])$ 
- $X$ is the Kummer surface of the product of two nonisogeneous elliptic curves $([64])$;

- $X$ is birationally isomorphic to the Hessian surface of a general cubic surface $([34])$;

- $S_{X}(-1)=U \perp E_{8}^{2} \perp A_{2}([115])$;

- $S_{X}(-1)=U \perp E_{8}^{2} \perp A_{1}^{2}([115])$;

- $S_{X}$ is of rank 20 with discriminant 7 ([13]);

- $S_{X}(-1)=U \perp D_{20}([35])$ (characteristic 2).

What is common about these examples is that the lattice $S_{X}(-1)$ can be primitively embedded in the lattice $I I_{25,1}$ from Example 4.6 as an orthogonal sublattice to a finite root sublattice of $I I_{25,1}$. We refer to [13] for the most general method for describing $\operatorname{Aut}(X)$ in this case.

5.4. Enriques surfaces. These are the surfaces from Case 2 (ii). They satisfy

$$
2 c_{1}(X)=0, \quad c_{1}(X) \neq 0, \quad H_{1}(X, \mathbb{Z})=\operatorname{Tors}\left(H^{2}(X, \mathbb{Z})\right)=\mathbb{Z} / 2 \mathbb{Z} .
$$

The cover $Y \rightarrow X$ corresponding to the generator of $H_{1}(X, \mathbb{Z})$ is a K3 surface. So, Enriques surfaces correspond to pairs $(Y, \tau)$, where $Y$ is a $\mathrm{K} 3$ surface and $\tau$ is its fixed-point-free involution.

We have

$$
S_{X}(-1)=U \perp E_{8} \cong E_{10} .
$$

The reflection group of $E_{10}$ is the group $W(2,3,7)$. Since its fundamental polytope does not have nontrivial symmetries, we obtain from (4.5)

$$
\mathrm{O}\left(S_{X}\right)^{+}=\operatorname{Ref}\left(S_{X}(-1)\right) \cong W(2,3,7)
$$

Let $W_{X}^{+}$be the subgroup of $\mathrm{O}\left(S_{X}\right)$ generated by reflections $r_{\alpha}$, where $\alpha$ is the image in $S_{X}$ of the cohomology class of a $(-2)$-curve on $X$. The following theorem follows (but nontrivially) from the Global Torelli Theorem for K3 surfaces ([83], [84]).

Theorem 5.12. Let $A_{X}$ be the image of Aut $(X)$ in $\mathrm{O}\left(S_{X}\right)$. Then $A_{X} \subset \mathrm{O}\left(S_{X}\right)^{+}$, its intersection with $W_{X}^{+}$is trivial and $W_{X}^{+} \rtimes A_{X}$ is of finite index in $\mathrm{O}\left(S_{X}\right)$.

This gives as a corollary that $\operatorname{Aut}(X)$ is finite if and only if $W_{X}^{+}$is of finite index in $\mathrm{O}\left(S_{X}\right)$. A general Enriques surface (in some precise meaning) does not contain $(-2)$-curves, so $A(X)$ is isomorphic to a subgroup of finite index of the orthogonal group $\mathrm{O}\left(E_{10}\right)$. In fact, more precisely, the group $\operatorname{Aut}(X)$ is isomorphic to the 2level congruence subgroup of $W\left(E_{10}\right)$ defined in (5.10) (see [8], [84]). The fact that the automorphism group of a general Enriques surface and a general Coble surface are isomorphic is not a coincidence, but I am not going to explain it here (see [31]).

All Enriques surfaces $X$ with finite $\operatorname{Aut}(X)$ were classified by S. Kondō [68] and (not constructively) by V. Nikulin [85]. In [30] I gave an example of an Enriques surface with finite automorphism group, believing that it was the first example of this kind. After the paper had been published I found that the existence of another example was claimed much earlier by G. Fano [43]. However, his arguments are very obscure and impossible to follow. The Coxeter diagram of the reflection group $W_{X}^{+}$in my example is given in Figure 13. 


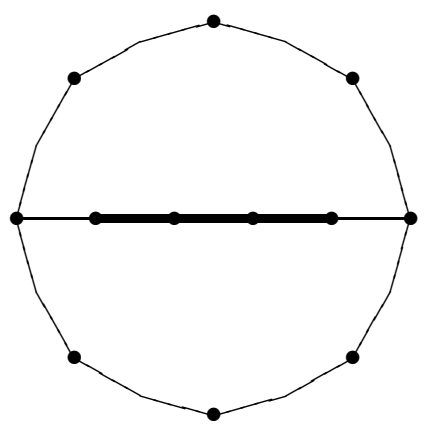

FIGURE 13

\section{Cremona transformations}

6.1. Plane Cremona transformations. A Cremona transformation of projective space $\mathbb{P}^{n}$ (as always over complex numbers) is a birational map of algebraic varieties. It can be given in projective coordinates by $n+1$ homogeneous polynomials of the same degree $d$ :

$$
T:\left(t_{0}, \ldots, t_{n}\right) \mapsto\left(P_{0}\left(t_{0}, \ldots, t_{n}\right), \ldots, P_{n}\left(t_{0}, \ldots, t_{n}\right)\right) .
$$

Dividing by a common multiple of the polynomials we may assume that the map is not defined on a closed subset of codimension $\geq 2$, the set of common zeros of the polynomials $P_{0}, \ldots, P_{n}$. Let $U=\operatorname{dom}(T)$ be the largest open subset where $T$ is defined and let $X$ be the Zariski closure of the graph of $T: U \rightarrow \mathbb{P}^{n}$ in $\mathbb{P}^{n} \times \mathbb{P}^{n}$. By considering the two projections of $X$, we get a commutative diagram of birational maps:

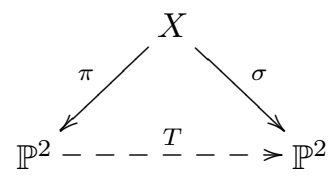

By taking a resolution of singularities $X^{\prime} \rightarrow X$, we may assume that such a diagram exists with $X$ nonsingular. We call it a resolution of indeterminacy points of $T$.

We denote the projective space of homogeneous polynomials of degree $d$ in $n+1$ variables by $\left|\mathcal{O}_{\mathbb{P}^{n}}(d)\right|$. The projective subspace of dimension $n$ spanned by the polynomials $P_{0}, \ldots, P_{n}$ defining the map $T$ is denoted by $L(T)$ and is called the linear system defining $T$. It depends only on $T$ (recall that two birational maps are equal if they coincide on a Zariski open subset). Any $n$-dimensional projective subspace $L$ of $\left|\mathcal{O}_{\mathbb{P}^{n}}(d)\right|$ (linear system) which consists of polynomials without a common factor defines a rational map $\mathbb{P}^{n}-\rightarrow \mathbb{P}^{n}$ by simply choosing a linear independent ordered set of $n+1$ polynomials from $L$. When the map happens to be birational, the linear system is called homaloidal. One obtains $L(T)$ as follows. First one considers the linear system $\left|\mathcal{O}_{\mathbb{P}^{n}}(1)\right|$ of hyperplanes in the target $\mathbb{P}^{n}$. The pre-image of its member on $X$ under the map $\sigma$ is a hypersurface on $X$; we push it down by $\pi$ and get a hypersurface on the domain $\mathbb{P}^{n}$. The set of such hypersurfaces forms a linear system $L(T)$. 
Now let us assume that $n=2$. As in section 5.2 we consider a factorization (5.4) of $\pi$,

$$
\pi: X=X_{N} \stackrel{\pi_{N}}{\longrightarrow} X_{N-1} \stackrel{\pi_{N-1}}{\longrightarrow} \ldots \stackrel{\pi_{2}}{\longrightarrow} X_{1} \stackrel{\pi_{1}}{\longrightarrow} X_{0}=\mathbb{P}^{2},
$$

where $\pi_{k+1}$ is the blow-up of a point $x_{k} \in X_{k}, k=0, \ldots, N-1$. Recall that the multiplicity $\operatorname{mult}_{x}(D)$ of a hypersurface $D$ at a point $x$ on a nonsingular variety is the degree of the first nonzero homogeneous part in the Taylor expansion of its local equation at $x$. Define inductively the numbers $m_{i}$ as follows. Let $L=L(T)$ be the linear system of curves on $\mathbb{P}^{2}$ defining $T$. First we set

$$
m_{1}=\min _{D \in L} \operatorname{mult}_{x_{1}} D .
$$

The linear system $\pi_{1}^{*}(L)$ on $X_{1}$ which consists of the full pre-images of hypersurfaces from $L(T)$ on $X_{1}$ has the hypersurface $m_{1} E_{1}$ as a fixed component. Let

$$
L_{1}=\pi_{1}^{*}(L)-m_{1} E_{1} .
$$

This is a linear system on $X_{1}$ without fixed components. Suppose $m_{1}, \ldots, m_{i}$ and $L_{1}, \ldots, L_{i}$ have been defined. Then we set

$$
\begin{gathered}
m_{i+1}=\min _{D \in L_{i}} \operatorname{mult}_{x_{i+1}} D, \\
L_{i+1}=\pi_{i+1}^{*}\left(L_{i}\right)-m_{i} E_{i+1} .
\end{gathered}
$$

It follows from the definition that

$$
L_{N}=\pi^{*}(L)-\sum_{i=1}^{N} m_{i} \mathcal{E}_{i}
$$

has no fixed components and is equal to the pre-image of $\left|\mathcal{O}_{\mathbb{P}^{2}}(1)\right|$ under $\sigma$. The image of $L_{N}$ in $\mathbb{P}^{2}$ is equal to the linear system $L(T)$. It is denoted by

$$
\left|\mathcal{O}_{\mathbb{P}^{2}}(d)-m_{1} x_{1}-\ldots-m_{N} x_{N}\right| .
$$

The meaning of the notation is that $L(T)$ consists of plane curves of degree $d$ which pass through the points $x_{i}$ with multiplicities $\geq m_{i}$.

We have similar decomposition for the map $\sigma$ which defines the linear system $L\left(T^{-1}\right)$.

As we have shown in section 5.2, two factorizations of birational regular maps from $X$ to $\mathbb{P}^{2}$ in a sequence of blow-ups define two geometric bases of the lattice $S_{X}$. A Cremona transformation (6.1) together with a choice of a diagram (6.2) and the factorizations (5.4) for $\pi$ and $\sigma$ is called a marked Cremona transformation. It follows from the proof of Theorem 5.2 that any marked Cremona transformation defines an element of the Coxeter group $W\left(E_{N}\right)$. The corresponding matrix is called the characteristic matrix of the marked Cremona transformation.

Example 6.1. Let $T$ be the standard quadratic Cremona transformation defined by

$$
T:\left(x_{0}, x_{1}, x_{2}\right) \mapsto\left(x_{0}^{-1}, x_{1}^{-1}, x_{2}^{-1}\right)
$$

(to make sense of this one has to multiply all coordinates at the output by $x_{0} x_{1} x_{2}$ ). It is not defined at points $p_{1}=(1,0,0), p_{2}=(0,1,0), p_{3}=(0,0,1)$. Let $\pi: X=$ $X_{3} \rightarrow X_{2} \rightarrow X_{1} \rightarrow X_{0}=\mathbb{P}^{2}$ be the composition of the blow-up of $p_{1}$, then the blow-up of the pre-image of $p_{2}$, and finally the pre-image of $p_{3}$. It is easy to see on the open subset $U$ where $T$ is defined the coordinate line $t_{i}=0$ is mapped to the point $p_{i}$. This implies that the Zariski closures on $X$ of the pre-images of 
intersections of these lines with $U$ are the curves $E_{1}^{\prime}, E_{2}^{\prime}, E_{3}^{\prime}$ on $X$ which are blown down to the points $p_{1}, p_{2}, p_{3}$ under $T$. The factorization for $\sigma: X \rightarrow \mathbb{P}^{2}$ could be chosen in such a way that $E_{i}^{\prime}=\mathcal{E}_{i}^{\prime}$ are the exceptional curves. These curves define a new geometric basis in $X$ with

$$
e_{1}^{\prime}=e_{0}-e_{2}-e_{3}, \quad e_{1}^{\prime}=e_{0}-e_{1}-e_{3}, \quad e_{1}^{\prime}=e_{0}-e_{0}-e_{1} .
$$

Since $k_{X}=-3 e_{0}+e_{1}+e_{2}=e_{3}=-3 e_{0}^{\prime}+e_{1}^{\prime}+e_{2}^{\prime}+e_{3}^{\prime}$, we also get $e_{0}^{\prime}=2 e_{0}^{\prime}-$ $e_{1}^{\prime}-e_{2}^{\prime}-e_{3}^{\prime}$. The corresponding transformation is the reflection with respect to the vector $e_{0}-e_{1}-e_{2}-e_{3}$. Given any set of 3 noncollinear points $q_{1}, q_{2}, q_{3}$, one can find a quadratic ${ }^{9}$ Cremona transformation $T^{\prime}$ with indeterminacy points $q_{1}, q_{2}, q_{3}$. For this we choose a projective transformation $g$ which sends $q_{i}$ to $p_{i}$ and take $T^{\prime}=T \circ g$.

Theorem 6.2. Let $A$ be the matrix representing an element from $W\left(E_{N}\right)$. Then there exists a marked Cremona transformation whose characteristic matrix is equal to $A$.

Proof. Let $A$ be the matrix of $w \in W\left(E_{N}\right)$ with respect to the standard basis $\mathbf{e}_{0}, \ldots, \mathbf{e}_{N}$ of $I_{1, N}$. Its first column is a vector $\left(m_{0},-m_{1}, \ldots,-m_{N}\right)$. Write $w$ as a word in reflections $r_{\mathbf{a}_{i}}$ and use induction on the length of $w$ to prove the Noether inequalities $m_{i} \geq 0, i \geq 1$ and $m_{0}>3 \max \left\{m_{i}, i \geq 1\right\}$. Now, let us use induction on the length of $w$ to show that the linear system $\mid \mathcal{O}_{\mathbb{P}^{2}}\left(m_{0}\right)-m_{1} p_{1}-$ $\left.\ldots-m_{N} p_{N}\right) \mid$ is homaloidal for some points $p_{1}, \ldots, p_{N}$ in general position. If $w$ is a simple reflection $r_{\mathbf{a}_{i}}$ we get $w\left(\mathbf{e}_{0}\right)=\mathbf{e}_{0}$ or $2 \mathbf{e}_{0}-\mathbf{e}_{1}-\mathbf{e}_{2}-\mathbf{e}_{3}$. In the first case the linear system defines a projective transformation, and in the second case it defines a standard quadratic transformation if we choose three noncollinear points $p_{1}, p_{2}, p_{3}$. Now write $w=r_{\mathbf{a}_{i}} w^{\prime}$ where the length of $w^{\prime}$ is less than the length of $w$. By induction, $w^{\prime}$ defines a homaloidal linear system $\mid \mathcal{O}_{\mathbb{P}^{2}}\left(m_{0}^{\prime}\right)-m_{1}^{\prime} p_{1}-\ldots-$ $\left.m_{N}^{\prime} p_{N}\right) \mid$ for some points $p_{1}, \ldots, p_{N}$ in general position. Let $\Phi^{\prime}: \mathbb{P}^{2}-\rightarrow \mathbb{P}^{2}$ be the corresponding Cremona transformation. If $i \neq 0$, the reflection permutes the $m_{i}^{\prime}, i>0$, and the linear system is still homaloidal and the set of points does not change. Since the points are in general position we may assume that $p_{1}, p_{2}, p_{3}$ are distinct noncollinear points. Composing $\Phi^{\prime}$ with a projective transformation we may assume that $p_{1}=(1,0,0), p_{2}=(0,1,0), p_{3}=(0,0,1)$. The computation from the previous example shows that $r_{\mathbf{a}_{0}} w^{\prime}\left(e_{0}\right)=m_{0} \mathbf{e}_{0}-\sum_{i>1} m_{i} \mathbf{e}_{i}$, where $m_{i}=$ $2 m_{i}^{\prime}-m_{1}-m_{2}-m_{3}, i \leq 3$, and $m_{i}=m_{i}^{\prime}, i>3$. Let $\Phi=\Phi^{\prime} \circ T$, where $T$ is the standard quadratic transformation discussed in the previous example. Then it is easy to see that $\Phi$ is given by the linear system $\left.\mid \mathcal{O}_{\mathbb{P}^{2}}\left(m_{0}\right)-m_{1} p_{1}-\ldots-m_{N} p_{N}\right) \mid$. Let $\tilde{w} \in W\left(E_{N}\right)$ correspond to the characteristic matrix of $\Phi$ (with respect to an appropriate marking). We have proved that $w\left(\mathbf{e}_{0}\right)=\tilde{w}\left(\mathbf{e}_{0}\right)$. This implies that $\tilde{w} w^{-1}\left(\mathbf{e}_{0}\right)=\mathbf{e}_{0}$. The matrix representing an element from $\mathrm{O}(1, N)$ whose first column is the unit vector is a diagonal matrix with \pm 1 at the diagonal. As we have seen already in the proof of Theorem 5.2, this implies that $\tilde{w}=w$.

One can apply Theorem 6.2 to list the types $\left(m_{0}, m_{1}, \ldots, m_{N}\right)$ of all homaloidal linear systems with $N$ indeterminacy points. They correspond to the orbit of the vector $e_{0}$ with respect to the group $W\left(E_{N}\right)$. In particular, the number of types is finite only for $N \leq 8$.

\footnotetext{
${ }^{9}$ i.e. defined by polynomials of degree 2 .
} 
6.2. Cremona action of $W(p, q, r)$. Consider the natural diagonal action of the group $G=\operatorname{PGL}(n+1, \mathbb{C})$ on $\left(\mathbb{P}^{n}\right)^{N}$, where $m=N-n-2 \geq 0$. A general orbit contains a unique point set $\left(p_{1}, \ldots, p_{N}\right)$ with the first $n+2$ points equal to the set of reference points $(1,0, \ldots, 0), \ldots,(0, \ldots, 0,1),(1, \ldots, 1)$. This easily implies that the field of $G$-invariant rational functions on $\left(\mathbb{P}^{n}\right)^{N}$ is isomorphic to the field of rational functions on $\left(\mathbb{P}^{n}\right)^{m}$ and hence is isomorphic to the field of rational functions $\mathbb{C}\left(z_{1}, \ldots, z_{n m}\right)$. The symmetric group $\Sigma_{N}$ acts naturally on this field via its action on $\left(\mathbb{P}^{n}\right)^{N}$ by permuting the factors. Assume $n \geq 2$ and consider $\Sigma_{N}$ as a subgroup $W(1, n+2, m+1)$ of the Coxeter group of type $W(2, n+1, m+1)$ corresponding to the subdiagram of type $A_{N-1}$ of the Coxeter diagram of $W(2, n+1, m+1)$. In 1917 A. Coble extended the action of $\Sigma_{N}$ on the field $\mathbb{C}\left(z_{1}, \ldots, z_{n m}\right)$ to the action of the whole group $W(2, n+1, m+1)$. This construction is explained in modern terms in [36]. In Coble's action the remaining generator of the Coxeter group acts as a standard quadratic transformation $\mathbb{P}^{n}-\rightarrow \mathbb{P}^{n}$ defined by

$$
T:\left(x_{0}, \ldots, x_{n}\right) \mapsto\left(x_{0}^{-1}, \ldots, x_{n}^{-1}\right) .
$$

One takes a point set $\left(p_{1}, \ldots, p_{N}\right)$, where the first $n+2$ points are the reference points, then applies $T$ to the remaining points to get a new set,

$$
\left(p_{1}, \ldots, p_{n+1}, T\left(p_{n+2}\right), \ldots, T\left(p_{N}\right)\right) .
$$

The Cremona action is the corresponding homomorphism of groups

$$
W(2, n+1, m+1) \rightarrow \operatorname{Aut}_{\mathbb{C}}\left(\mathbb{C}\left(z_{1}, \ldots, z_{n m}\right)\right) .
$$

One can show that for $N \geq 9$, this homomorphism does not arise from a regular action of the Coxeter group on any Zariski open subset of $\left(\mathbb{P}^{n}\right)^{m}$.

The following result of S. Mukai [82] extends the Cremona action to any group $W(p, q, r)$.

Theorem 6.3. Let $X=\left(\mathbb{P}^{q-1}\right)^{p-1}$. Consider the natural diagonal action of the group $\operatorname{PGL}(q, \mathbb{C})^{p-1}$ on $X$ and extend it to the diagonal action on $X_{p, q, r}:=X^{q+r}$. Let $K(p, q, r)$ be the field of invariant rational functions on $X_{p, q, r}$ isomorphic to $\mathbb{C}\left(t_{1}, \ldots, t_{d}\right), d=(p-1)(q-1)(r-1)$. Then there is a natural homomorphism,

$$
c r_{p, q, r}: W(p, q, r) \rightarrow \operatorname{Aut}_{\mathbb{C}}(K(p, q, r)) .
$$

It coincides with the Coble action when $p=2$.

It seems that the homomorphism $\mathrm{cr}_{p, q, r}$ is always injective when the group is infinite. The geometric meaning of the kernels in the case of finite groups $W(2, q, r)$ are discussed in [36] and [39].

The reflections corresponding to the vertices on the branches of the $T_{p, q, r^{-}}$ diagram with $q$ and $r$ vertices act by permuting the factors of $X$. The reflections corresponding to $p-2$ last vertices of the $p$-branch permute the factors of $X$. The second vertex on the $p$-branch acts via the Cremona transformation in $\left(\mathbb{P}^{q-1}\right)^{p-1}$,

$$
\left(\left(x_{0}^{(1)}, \ldots, x_{q-1}^{(1)}\right), \ldots,\left(x_{0}^{(p-1)}, \ldots, x_{q-1}^{(p-1)}\right)\right) \rightarrow\left(\left(\frac{1}{x_{0}^{(1)}}, \ldots, \frac{1}{x_{q-1}^{(1)}}\right), \ldots,\left(\frac{x_{0}^{(p-1)}}{x_{0}^{(1)}}, \ldots, \frac{x_{q-1}^{(p-1)}}{x_{q-1}^{(1)}}\right)\right) .
$$

Let $Y(p, q, r)$ be a birational model of the field $K(p, q, r)$ on which $W(p, q, r)$ acts birationally via $c_{p q r}$. For any $g \in W(p, q, r)$ let $\operatorname{dom}(g)$ be the domain of definition of $g$. Let $Z$ be a closed irreducible subset of $Y(p, q, r)$ with generic point $\eta_{Z}$. Let

$$
G_{Z}=\left\{g \in W(p, q, r): \eta_{Z} \in \operatorname{dom}(g) \cap \operatorname{dom}\left(g^{-1}\right), g\left(\eta_{Z}\right)=\eta_{Z}\right\}
$$


be the decomposition subgroup of $Z$ in $W(p, q, r)$ and $G_{Z}^{i}$ be the inertia subgroup of $Z$, the kernel of the natural map $G_{Z} \rightarrow \operatorname{Aut}(R(Z))$, where $R(Z)$ is the field of rational functions of $Z$. These groups were introduced for any group of birational transformations by M. Gizatullin [47]. Define a $\operatorname{PGL}(q, \mathbb{C})^{p-1}$-invariant closed irreducible subset $S$ of $X_{p, q, r}$ to be special if it defines a closed subset $Z$ on some birational model $Y(p, q, r)$ such that $G_{Z}=W(p, q, r)$ and $G_{Z}^{i}$ is a subgroup of finite index of $W(p, q, r)$.

Consider a general point $s \in S$ as a set of $q+r$ distinct points in $\left(\mathbb{P}^{q-1}\right)^{p-1}$ and let $V(s) \rightarrow\left(\mathbb{P}^{q-1}\right)^{p-1}$ be the blow-up of this set. One can show that the group $G_{Z}^{\prime}$ is realized as a group of pseudo-automorphisms of $V(s) .{ }^{10}$

I know only a few examples of special subsets when $W(p, q, r)$ is infinite. Here are some examples.

- $(p, q, r)=(2,3,6), S$ parametrizes ordered sets of base points of a pencil of plane cubic curves in $\mathbb{P}^{2}[20],[36]$;

- $(p, q, r)=(2,4,4), S$ parametrizes ordered sets of base points of a net of quadrics in $\mathbb{P}^{3}[20],[36]$;

- $(p, q, r)=(2,3,7), S$ parametrizes ordered sets of double points of a rational plane sextic [20], [36];

- $(p, q, r)=(2,4,6), S$ parametrizes ordered sets of double points of a quartic symmetroid surface [20], [24].

The inertia subgroups of finite index of $W(p, q, r)$ defined in these examples have the quotient groups isomorphic to simple groups $\mathrm{O}\left(8, \mathbb{F}_{2}\right)^{+}, \mathrm{Sp}\left(6, \mathbb{F}_{2}\right), \mathrm{O}\left(10, \mathbb{F}_{2}\right)^{+}$ and $\operatorname{Sp}\left(8, \mathbb{F}_{2}\right)$, respectively.

Remark 6.4. It is popular in group theory to represent a sporadic simple group or a related group as a finite quotient of a Coxeter group $W(p, q, r)$. For example, the Monster group $F_{1}$ is a quotient of $W(4,5,5)$. The Bimonster group $F_{1} 22$ is a quotient of $W(6,6,6)$ by a single relation [61], [88]. Is there a geometric interpretation of these presentations in terms of the Cremona action of $W(p, q, r)$ on some special subset of points in $X_{p, q, r}$ ? Mukai's construction should relate the Monster group with some special configurations of 10 points in $\left(\mathbb{P}^{3}\right)^{4}$ or 9 points in $\left(\mathbb{P}^{4}\right)^{4}$. The Bimonster group could be related to special configurations of 12 points in $\left(\mathbb{P}^{5}\right)^{5}$ (see related speculations in [3]).

\section{INVARIANTS OF FINITE COMPLEX REFLECTION GROUPS}

Let $\Gamma \subset \operatorname{GL}(n+1, \mathbb{C})$ be a finite linear complex reflection group in $\mathbb{C}^{n+1}$ and let $\bar{\Gamma}$ be its image in $\operatorname{PGL}(n, \mathbb{C})$. The reflection hyperplanes of $G$ define a set of hyperplanes in $\mathbb{P}^{n}$ and the zeroes of $G$-invariant polynomials define hypersurfaces in $\mathbb{P}^{n}$. The geometry, algebra, combinatorics and topology of arrangements of reflection hyperplanes of finite complex reflection groups is a popular area in the theory of hyperplane arrangements (see [89], [90]). On the other hand, classical algebraic geometry is full of interesting examples of projective hypersurfaces whose symmetries are described in terms of a complex reflection group. We discuss only a few examples.

We begin with the group $J_{3}(4)$ of order 336 (No. 24 in the list). It has fundamental invariants of degrees 4,6 and 14 . Its center is of order 2 , and the group $\bar{\Gamma}$

\footnotetext{
${ }^{10} \mathrm{~A}$ pseudo-automorphism is a birational transformation which is an isomorphism outside a closed subset of codimension $>2$.
} 
is a simple group of order 168 isomorphic to $\operatorname{PSL}\left(2, \mathbb{F}_{7}\right)$. The invariant curve of degree 4 is of course the famous Klein quartic, which is projectively equivalent to the curve

$$
F_{4}=T_{0}^{3} T_{1}+T_{1}^{3} T_{2}+T_{2}^{3} T_{0}=0 .
$$

There are 21 reflection hyperplanes in $\mathbb{P}^{2}$. They intersect the curve at 84 points, forming an orbit with stabilizer subgroups of order 2 . The invariant $F_{6}$ of degree 6 defines a nonsingular curve of degree 6 , the Hessian curve of the Klein quartic. Its equation is given by the Hesse determinant of second partial derivatives of $F_{4}$.

The double cover of $\mathbb{P}^{2}$ branched along the curve $F_{6}=0$ is a K3 surface $X$. The automorphism group of $X$ is an infinite group which contains a subgroup isomorphic to $\bar{\Gamma}$.

Next we consider the group $L_{3}$ of order 648 (No. 25). The group $\bar{\Gamma}$ is of order 216 and is known as the Hessian group. ${ }^{11}$ It is isomorphic to the group of projective transformations leaving invariant the Hesse pencil of plane cubic curves,

$$
\lambda\left(t_{0}^{3}+t_{1}^{3}+t_{2}^{3}\right)+\mu t_{0} t_{1} t_{2}=0 .
$$

It is known that any nonsingular plane cubic curve is projectively isomorphic to one of the curves in the pencil. The base points of the pencil (i.e. points common to all curves from the pencil) are inflection points of each nonsingular member from the pencil. The singular members of the pencil correspond to the values of the parameters $(\lambda, \mu)=(0,1),(1,-3),\left(1,-3 e^{2 \pi i / 3}\right),\left(1,-3 e^{-2 \pi i / 3}\right)$. The corresponding cubic curves are the unions of 3 lines; all together we get 12 lines which form the 12 reflection hyperplanes.

The smallest degree invariant of $L_{3}$ in $\mathbb{C}^{4}$ is a polynomial of degree 6 :

$$
F_{6}=T_{0}^{6}+T_{1}^{6}+T_{2}^{6}-10\left(T_{0}^{3} T_{1}^{3}+T_{0}^{3} T_{2}^{3}+T_{1}^{3} T_{2}^{3}\right) .
$$

The double cover of $\mathbb{P}^{2}$ branched along the curve $F_{6}=0$ is a $\mathrm{K} 3$ surface. Its group of automorphisms is an infinite group containing a subgroup isomorphic to the Hesse group.

Next we turn our attention to complex reflection groups of types $K_{5}, L_{4}$ and $E_{6}$. Their orders are all divisible by $6 ! \cdot 36=25,920$ equal to the order of the simple group $\operatorname{PSp}\left(4, \mathbb{F}_{3}\right)$. The group of type $E_{6}$ (the Weyl group of the lattice $E_{6}$ ) contains this group as a subgroup of index 2 , which consists of words in fundamental reflections of even length. The group $K_{5}$ (No. 35) is the direct product of $\operatorname{PSp}\left(4, \mathbb{F}_{3}\right)$ and a group of order 2. The group $L_{4}$ (No. 32) is the direct product of a group of order 3 and $\operatorname{Sp}\left(4, \mathbb{F}_{3}\right)$.

Let $\Gamma$ be of type $K_{5}$. It acts in $\mathbb{P}^{4}$ with 45 reflection hyperplanes. The hypersurface defined by its invariant of degree 4 is isomorphic to the Burkhardt quartic in $\mathbb{P}^{4}$. Its equation can be given in more symmetric form in $\mathbb{P}^{5}$ :

$$
\sum_{i=0}^{5} T_{i}=\sum_{i=0}^{5} T_{i}^{4}=0 .
$$

These equations exhibit the action of the symmetric group $S_{6}$ contained in $\bar{\Gamma}$. It is easy to see that the hypersurface has 45 ordinary double points. This is a record for hypersurfaces of degree $4 \mathrm{in} \mathbb{P}^{4}$, and this property characterizes Burkhardt quartics. The dual representation of $\Gamma$ is a linear reflection representation too; it is obtained from the original one by composing it with an exterior automorphism of

\footnotetext{
${ }^{11}$ Not to be confused with the Hesse group related to 28 bitangents of a plane quartic.
} 
the group. The double points correspond to reflection hyperplanes in the dual space. The reflection hyperplanes in the original space cut out the Burkhardt quartic in special quartic surfaces (classically known as desmic quartic surfaces). They are birationally isomorphic to the Kummer surface of the product of an elliptic curve with itself.

Finally note that the Burkhardt quartic is a compactification of the moduli space of principally polarized abelian surfaces with level 3 structure. All of this and much much more can be found in [59].

Let $\Gamma$ be of type $L_{4}$. The number of reflection hyperplanes is 40 . The stabilizer subgroup of each hyperplane is the group $L_{3}$ from above. The smallest invariant is of degree 12. The corresponding hypersurface cuts out in each reflection hyperplane the 12 reflection lines of the Hessian group. Again for more of this beautiful geometry we refer to Hunt's book, in which also the geometry of the Weyl group $W\left(E_{6}\right)$ in $\mathbb{P}^{5}$ is fully discussed. He calls the invariant hypersurface of degree 5 the gem of the universe.

\section{Monodromy GRoups}

8.1. Picard-Lefschetz transformations. Let $f: X \rightarrow S$ be a holomorphic map of complex manifolds which is a locally trivial $C^{\infty}$-fibration. One can construct a complex local coefficient system whose fibres are the cohomology with compact support $H_{c}^{n}\left(X_{s}, \Lambda\right)$ with some coefficient group $\Lambda$ of the fibres $X_{s}=f^{-1}(s)$. The local coefficent system defines the monodromy map

$$
\rho_{s_{0}}: \pi_{1}\left(S, s_{0}\right) \rightarrow \operatorname{Aut}\left(H_{c}^{n}\left(X_{s_{0}}, \Lambda\right)\right) .
$$

We will be interested in the cases when $\Lambda=\mathbb{Z}, \mathbb{R}$, or $\mathbb{C}$ that lead to integral, real or complex monodromy representations.

The image of the monodromy representation is called the monodromy group of the map $f$ (integral, real, complex).

We refer to [7], [53], [76] for some of the material which follows.

Let $f: \mathbb{C}^{n+1} \rightarrow \mathbb{C}$ be a holomorphic function with an isolated critical point at $x_{0}$. We will be interested only in germs $\left(f, x_{0}\right)$ of $f$ at $x_{0}$. Without loss of generality we may assume that $x_{0}$ is the origin and $f\left(x_{0}\right)=0$. The level set $V=f^{-1}(0)$ is an analytic subspace of $\mathbb{C}^{n+1}$ with isolated singularity at 0 . The germ of $(V, 0)$ is an $n$-dimensional isolated hypersurface singularity. In general the isomorphism type of the germ of $(V, 0)$ does not determine the isomorphism type of the germ $(f, 0)$. However, it does in one important case when $f$ is a weighted homogeneous polynomial. ${ }^{12}$ In this case we say that the germ $(V, 0)$ is a weighted homogeneous isolated hypersurface singularity.

It was shown by J. Milnor [79] that for sufficiently small $\epsilon$ and $\delta$, the restriction of $f$ to

$$
X=\left\{z \in \mathbb{C}^{n+1}:\|z\|<\epsilon, 0<|f(z)|<\delta\right\}
$$

is a locally trivial $C^{\infty}$ fibration whose fibre is an open $n$-dimensional complex manifold. Moreover, each fibre has the homotopy type of a bouquet of $n$-spheres. The number $\mu$ of the spheres is equal to the multiplicity of $f$ at 0 computed as the

\footnotetext{
${ }^{12}$ This means that $f\left(z_{1}, \ldots, z_{n+1}\right)$ is a linear combination of monomials of the same degree, where each variable is weighted with some positive number.
} 
dimension of the jacobian algebra

$$
J_{f}=\operatorname{dim}_{\mathbb{C}} \mathbb{C}\left[\left[z_{1}, \ldots, z_{n+1}\right]\right] /\left(\frac{\partial f}{\partial z_{1}}, \ldots, \frac{\partial f}{\partial z_{n+1}}\right) .
$$

Let $D_{\epsilon}^{*}=\{t \in \mathbb{C}: 0<|t|<\epsilon\}$ and $f: X \rightarrow D_{\delta}^{*}$ be the above fibration, a Milnor fibration of $(V, 0)$. Let

$$
M_{t}=H_{c}^{n}\left(F_{t}, \mathbb{Z}\right) \cong H_{n}\left(F_{t}, \mathbb{Z}\right)
$$

The bilinear pairing

$$
H_{c}^{n}\left(F_{t}, \mathbb{Z}\right) \times H_{c}^{n}\left(F_{t}, \mathbb{Z}\right) \rightarrow H_{c}^{2 n}\left(F_{t}, \mathbb{Z}\right) \cong \mathbb{Z}
$$

is symmetric if $n$ is even and skew-symmetric otherwise. To make the last isomorphism unique we fix an orientation on $F_{t}$ defined by the complex structure on the open ball $B_{\epsilon}=\left\{z \in \mathbb{C}^{n+1}:\|z\|<\epsilon\right\}$. Thus, if $n$ is even, which we will assume from now on, the bilinear pairing equips $M_{t}$ with a structure of a lattice, called the Milnor lattice. Its isometry class does not depend on the choice of the point $t$ in $\pi_{1}\left(D_{\delta}^{*} ; t_{0}\right)$. Fixing a point $t_{0} \in D_{\epsilon}^{*}$ we obtain the classical monodromy map

$$
\pi_{1}\left(D_{\epsilon}^{*} ; t_{0}\right) \cong \mathbb{Z} \rightarrow \mathrm{O}\left(M_{t_{0}}\right) .
$$

Choosing a generator of $\pi_{1}\left(D_{\epsilon}^{*} ; t_{0}\right)$, one sees that the map defines an isometry of the Milnor lattice which is called a classical monodromy operator.

Example 8.1. Let

$$
q\left(z_{1}, \ldots, z_{n+1}\right)=z_{1}^{2}+\ldots+z_{n+1}^{2} .
$$

It has a unique critical point at the origin with critical value 0 . An isolated critical point $\left(f, x_{0}\right)$ (resp. isolated hypersurface singularity $\left(V, x_{0}\right)$ ) locally analytically isomorphic to $(q, 0)$ (resp. $\left.\left(q^{-1}(0), 0\right)\right)$ is called a nondegenerate critical point (resp. ordinary double point or ordinary node).

Let $\epsilon$ be any positive number and $\delta<\sqrt{\epsilon}$. For any $t \in D_{\delta}^{*}$, the intersection $F_{t}=q^{-1}(t) \cap B_{\epsilon}$ is nonempty and is given by the equations

$$
\sum_{i=1}^{n+1} z_{i}^{2}=t, \quad\|z\|<\epsilon .
$$

Writing $z_{i}=x_{i}+\sqrt{-1} y_{i}$ we find the real equations

$$
\|x\|^{2}-\|y\|^{2}=t, \quad x \cdot y=0, \quad\|x\|^{2}+\|y\|^{2}<\epsilon^{2} .
$$

After some smooth coordinate change, we get the equations

$$
\|x\|^{2}=1, \quad x \cdot y=0, \quad\|y\|^{2}<1 .
$$

It is easy to see that these are the equations of the open unit ball subbundle of the tangent bundle of the $n$-sphere $S^{n}$. Thus we may take $F_{t}$ to be a Milnor fibre of $(q, 0)$. The sphere $S^{n}$ is contained in $F_{t}$ as the zero section of the tangent bundle, and $F_{t}$ can be obviously retracted to $S^{n}$. Let $\alpha$ denote the fundamental class $\left[S^{n}\right]$ in $H_{c}^{n}\left(F_{t}, \mathbb{Z}\right)$. We have

$$
H_{c}^{n}\left(F_{t}, \mathbb{Z}\right)=\mathbb{Z} \alpha .
$$

Our orientation on $F_{t}$ is equal to the orientation of the tangent bundle of the sphere taken with the sign $(-1)^{n(n-1) / 2}$. This gives

$$
(\alpha, \alpha)=(-1)^{n(n-1) / 2} \chi\left(S^{n}\right)=(-1)^{n(n-1) / 2}\left(1+(-1)^{n}\right),
$$


and hence in the case when $n$ is even,

$$
(\alpha, \alpha)=\left\{\begin{array}{lll}
-2 & \text { if } n \equiv 2 \quad \bmod 4 \\
2 & \text { if } n \equiv 0 \quad \bmod 4 .
\end{array} .\right.
$$

By collapsing the zero section $S^{n}$ to the point, we get a map $F_{t}$ to $B_{\epsilon} \cap q^{-1}(0)$ which is a diffeomorphism outside $S^{n}$. For this reason the homology class $\delta$ is called the vanishing cycle.

The classical Picard-Lefschetz formula shows that the monodromy operator is a reflection transformation

$$
T(x)=x-(-1)^{n(n-1) / 2}(x, \delta) \delta .
$$

Let $(V, 0)$ be an isolated $n$-dimensional hypersurface singularity. A deformation of $(V, 0)$ is a holomorphic map-germ $\phi:\left(\mathbb{C}^{n+k}, 0\right) \rightarrow\left(\mathbb{C}^{k}, 0\right)$ with fibre $\phi^{-1}(0)$ isomorphic to $(V, 0)$. By definition $(V, 0)$ admits a deformation map $f:\left(\mathbb{C}^{n+1}, 0\right) \rightarrow$ $(\mathbb{C}, 0)$. Let $J_{f}$ be the jacobian algebra of $f(8.2)$ and

$$
J_{f=0}=J_{f} /(\bar{f}),
$$

where $\bar{f}$ is the coset of $f$ in $J_{f}$. Its dimension $\tau$ (the Tjurina number) is less than or equal to $\mu$. The equality occurs if and only if $(V, 0)$ is isomorphic to a weighted homogeneous singularity.

Choose a basis $z^{a_{1}}, \ldots, z^{a_{\tau}}$ of the algebra (8.6) represented by monomials with exponent vectors $a_{1}, \ldots, a_{\tau}$ with $a_{\tau}=0$ (this is always possible). Consider a deformation

$$
\Phi:\left(\mathbb{C}^{n+\tau}, 0\right) \rightarrow\left(\mathbb{C}^{\tau}, 0\right),(z, u) \rightarrow\left(f(z)+\sum_{i=1}^{\tau} u_{i} z^{a_{i}}, u_{1}, \ldots, u_{\tau-1}\right) .
$$

This deformation represents a semi-universal (miniversal) deformation of $(V, 0)$. Roughly speaking this means that any deformation $\phi:\left(\mathbb{C}^{n+k}, 0\right) \rightarrow\left(\mathbb{C}^{k}, 0\right)$ is obtained from $(8.7)$ by mapping $\left(\mathbb{C}^{k}, 0\right)$ to $\left(\mathbb{C}^{\tau}, 0\right)$ and taking the pull-back of the map $\phi$ (this explains the versal part). The map-germ $\left(\mathbb{C}^{k}, 0\right) \rightarrow\left(\mathbb{C}^{\tau}, 0\right)$ is not unique, but its derivative at 0 is unique (whence the semi in semi-universal).

Let

$$
\Delta=\left\{(t, u) \in \mathbb{C}^{\tau}: \Phi^{-1}(t, u) \text { has a singular point at some }(z, u)\right\} .
$$

Let $(\Delta, 0)$ be the germ of $\Delta$ at 0 . It is called the bifurcation diagram or discriminant of $f$. Choose a representative of $\Phi$ defined by restricting the map to some open ball $B$ in $\mathbb{C}^{n+\tau}$ with center at 0 and let $U$ be an open neighborhood of 0 in $\mathbb{C}^{\tau}$ such that $\pi^{-1}(U) \subset B$. When $B$ is small enough one shows that the restriction map

$$
\pi: \pi^{-1}(U \backslash U \cap \Delta) \rightarrow U \backslash \Delta
$$

is a locally trivial $C^{\infty}$-fibration with fibre diffeomorphic to a Milnor fibre $F$ of $(f, 0)$. Fixing a point $u_{0} \in U \backslash \Delta$, we get the global monodromy map of $(X, 0)$,

$$
\pi_{1}\left(U \backslash \Delta ; u_{0}\right) \rightarrow \mathrm{O}\left(H_{c}^{n}\left(\pi^{-1}\left(u_{0}\right), \mathbb{Z}\right)\right) \cong \mathrm{O}(M),
$$

where $M$ is a fixed lattice in the isomorphism class of Milnor lattices of $(f, 0)$.

One can show that the conjugacy class of the image of the global monodromy map (the global monodromy group of $(X, 0)$ ) does not depend on the choices of $B, U, u_{0}$. 
Theorem 8.2. The global monodromy group $\Gamma$ of an isolated hypersurface singularity of even dimension $n=2 k$ is a subgroup of $\operatorname{Ref}_{-2}(M)$ if $k$ is odd and $\operatorname{Ref}_{2}(M)$ otherwise.

Proof. This follows from the Picard-Lefschetz theory. Choose a point $\left(t_{0}, u^{(0)}\right) \in$ $\mathbb{C}^{\tau} \backslash \Delta$ close enough to the origin and pass a general line $u_{i}=c_{i}\left(t-t_{0}\right)+u_{i}^{(0)}$ through this point. The pre-image of this line in $\mathbb{C}^{n+\tau}$ is the 1-dimensional deformation $\mathbb{C}^{n+1} \rightarrow \mathbb{C}$ given by the function $t=\tilde{f}(z)$ implicitly defined by the equation

$$
f(z)+t\left(-1+\sum_{i=1}^{\tau} c_{i} z_{i}^{a_{i}}\right)+\sum_{i=1}^{\tau}\left(u_{i}^{(0)}-c_{i} t_{0}\right) z_{i}^{a_{i}}=0 .
$$

By choosing the line general enough, we may assume that all critical points of this function are nondegenerate and critical values are all distinct. Its fibre over the point $t=t_{0}$ is equal to the fibre of the function $f(z)+\sum_{i=1}^{\tau} u_{i}^{(0)} z_{i}^{a_{i}}$ over $t_{0}$. Since the semi-universal deformation is a locally trivial fibration over the complement of $\Delta$, all nonsingular fibres are diffeomorphic. It follows from the Morse theory that the number of critical points of $\tilde{f}$ is equal to the Milnor number $\mu$. Let $t_{1}, \ldots, t_{\mu}$ be the critical values. Let $S\left(t_{i}\right)$ be a small circle around $t_{i}$ and $t_{i}^{\prime} \in S_{i}$. The function $\tilde{f}$ defines a locally trivial $C^{\infty}$-fibration over $\mathbb{C} \backslash\left\{t_{1}, \ldots, t_{\mu}\right\}$ so that we can choose a diffeomorphism of the fibres:

$$
\phi_{i}: \tilde{f}^{-1}\left(t_{0}\right) \rightarrow \pi^{-1}\left(u_{0}\right) .
$$

The Milnor fibre $F_{i}$ of $\tilde{f}$ at $t_{i}$ is an open subset of $\tilde{f}^{-1}\left(t_{i}^{\prime}\right)$ and hence defines an inclusion of the lattices:

$$
H_{n}\left(F_{i}, \mathbb{Z}\right) \hookrightarrow H_{n}\left(\tilde{f}^{-1}\left(t_{i}^{\prime}\right), \mathbb{Z}\right) \cong H_{n}\left(\tilde{f}^{-1}\left(t_{0}\right), \mathbb{Z}\right) \cong H_{n}\left(\pi^{-1}\left(u_{0}\right), \mathbb{Z}\right) \cong M .
$$

The image $\delta_{i}$ of the vanishing cycle $\delta_{i}^{\prime}$ generating $H_{n}\left(F_{i}, \mathbb{Z}\right)$ in $M$ is called a vanishing cycle of the Milnor lattice $M$. The vanishing cycles $\left(\delta_{1}, \ldots, \delta_{\mu}\right)$ form a basis in $M$.

For each $t_{i}$ choose a path $\gamma_{i}$ in $\mathbb{C} \backslash\left\{t_{1}, \ldots, t_{\mu}\right\}$ which connects $t_{0}$ with $t_{i}^{\prime}$ and when continues along the circle $S\left(t_{i}\right)$ in counterclockwise fashion until returning to $t_{i}^{\prime}$ and going back to $t_{0}$ along the same path but in the opposite direction. The homotopy classes $\left[\gamma_{i}\right]$ of the paths $\gamma_{i}$ generate $\pi_{1}\left(\mathbb{C} \backslash\left\{t_{1}, \ldots, t_{\mu}\right\} ; t_{0}\right)$, the map

$$
s_{*}: \pi_{1}\left(\mathbb{C} \backslash\left\{t_{1}, \ldots, t_{\mu}\right\} ; t_{0}\right) \rightarrow \pi_{1}\left(U \backslash \Delta ; u_{0}\right)
$$

is surjective and the images $g_{i}$ of $\left[\gamma_{i}\right]$ under the composition of the monodromy map and $s_{*}$ generate the monodromy group $\Gamma$. Applying the Picard-Lefschetz formula (8.5) we obtain, for any $x \in M$,

$$
g_{i}(x)=x-(-1)^{n(n-1) / 2}\left(x, \delta_{i}\right) \delta_{i}, \quad i=1, \ldots, \mu .
$$

This shows that $\Gamma$ is generated by $\mu$ reflections in elements $\delta_{i}$ satisfying (8.4). This proves the claim.

Remark 8.3. It is known that for any isolated hypersurface singularity given by a weighted homogeneous polynomial $P$ with isolated critical point at 0 the Milnor lattice is isomorphic to $H_{c}^{n}\left(P^{-1}(\epsilon), \mathbb{Z}\right)$. In this case we can define the monodromy group as the image of the monodromy map

$$
\pi_{1}\left(\mathbb{C}^{\mu} \backslash \Delta ; u_{0}\right) \rightarrow \mathrm{O}\left(H_{c}^{n}\left(\pi^{-1}\left(u_{0}\right), \mathbb{Z}\right)\right)
$$

where $u_{0} \in \mathbb{C}^{\mu} \backslash \Delta$. 
One can relate the global monodromy group with the classical monodromy operator.

Theorem 8.4. Let $T$ be a classical monodromy operator and $s_{1}, \ldots, s_{\mu}$ be the reflections in $\mathrm{O}(M)$ corresponding to a choice of paths $\gamma_{i}$ as above. Then $\pm T$ is conjugate to the product $s_{1} \cdots s_{\mu}$.

The product $s_{1} \cdots s_{\mu}$ is an analog of a Coxeter element in the reflection group of $M$ (see Example 5.7). However, the Weyl group of $M$ is not a Coxeter group in general.

8.2. Surface singularities. Assume now that $n=2$. We will represent a surface singularity by an isolated singular point 0 of an affine surface $X \subset \mathbb{C}^{3}$.

Let $\pi: X^{\prime} \rightarrow X$ be a resolution of the singularity $(X, 0)$. This means that $X^{\prime}$ is a nonsingular algebraic surface and $\pi$ is a proper holomorphic map which is an isomorphism over $U=X \backslash\{0\}$. We may assume it to be minimal, i.e. does not contain $(-1)$-curves in its fibre over 0 . This assumption makes it unique up to isomorphism.

One defines the following invariants of $(X, 0)$. The first invariant is the genus $\delta p_{a}$ of $(X, 0)$. This is the dimension of the first cohomology space of $X^{\prime}$ with coefficient in the structure sheaf $\mathcal{O}_{X^{\prime}}$ of regular (or holomorphic) functions on $X^{\prime}$. In spite of $X^{\prime}$ not being compact, this space is finite-dimensional.

Our second invariant is the canonical class square $\delta K^{2}$. To define it we use that there is a rational differential 2-form on $X$ which has no poles or zeros on $X \backslash\{0\}$. Its extension to $X^{\prime}$ has divisor $D$ supported at the exceptional fibre. It represents an element $[D]$ in $H_{c}^{2}\left(X^{\prime}, \mathbb{Z}\right)$. Using the cup-product pairing we get the number $[D]^{2}$, which we take for $\delta K^{2}$.

Finally we consider the fibre $E=\pi^{-1}(0)$ of a minimal resolution. This is a (usually reducible) holomorphic curve on $X$. We denote its Betti numbers by $b_{i}$. For example, $b_{2}$ is the number of irreducible components of $E$.

Since $X^{\prime}$ is defined uniquely up to isomorphism, the numbers $\delta p_{a}, \delta K^{2}, b_{i}$ are well-defined.

Remark 8.5. The notations $\delta p_{a}$ and $\delta K^{2}$ are explained as follows. Suppose $Y$ is a projective surface of degree $d$ in $\mathbb{P}^{3}$ with isolated singularities $y_{1}, \ldots, y_{k}$. Let $Y^{\prime}$ be its minimal resolution. Define the arithmetic genus of a nonsingular projective surface $V$ as $p_{a}=-q+p_{g}$, where $q=\operatorname{dim} H^{1}\left(V, \mathcal{O}_{V}\right)=\operatorname{dim} \Omega^{1}(X)$ is the dimension of the space of holomorphic 1-forms on $V$ and $p_{g}=\operatorname{dim} H^{2}\left(V, \mathcal{O}_{V}\right)=\operatorname{dim} \Omega^{2}(X)$ is the dimension of the space of holomorphic 2 -forms on $V$. Let $F_{d}$ be a nonsingular hypersurface of degree $d$ in $\mathbb{P}^{3}$. Then

$$
p_{a}\left(Y^{\prime}\right)=p_{a}\left(F_{d}\right)-\sum_{i=1}^{k} \delta p_{a}\left(x_{i}\right), \quad K_{Y^{\prime}}^{2}=K_{F_{d}}^{2}-\sum_{i=1}^{k} \delta K^{2}\left(x_{i}\right) .
$$

The numbers $p_{a}\left(F_{d}\right)$ and $K_{F_{d}}^{2}$ are easy to compute. We have

$$
p_{a}\left(F_{d}\right)=p_{g}\left(F_{d}\right)=(d-1)(d-2)(d-3) / 6, \quad K_{F_{d}}^{2}=d(d-4)^{2} .
$$

Theorem 8.6 (J. Steenbrink [108]). Let $\mu$ be the Milnor number of a surface singularity $(V, 0)$. Then the signature $\left(\mu_{+}, \mu_{-}, \mu_{0}\right)$ of the Milnor lattice is given as follows:

$$
\mu_{0}=b_{1}, \quad \mu_{+}+\mu_{-}+\mu_{0}=\mu, \quad \mu_{+}-\mu_{-}=-\delta K^{2}-b_{2}-8 \delta p_{a} .
$$


Example 8.7. The following properties are equivalent:

- $\delta p_{a}=0$

- $\mu_{+}=\mu_{0}=0$;

- $\delta K^{2}=0$

- $b_{2}=\mu$

- the exceptional curve of a minimal resolution is the union of nodal curves;

- $V$ can be given by equation $P\left(z_{1}, z_{2}, z_{3}\right)=0$, where $P$ is a weighted homogeneous polynomial of degree $d$ with respect to positive weights $q_{1}, q_{2}, q_{3}$ such that $d-q_{1}-q_{2}-q_{3}<0$;

- $(V, 0)$ is isomorphic to an affine surface with ring of regular functions isomorphic to the ring of invariant polynomials of a finite subgroup $G \subset \mathrm{SL}(2, \mathbb{C})$.

Table 3 gives the list of isomorphism classes of surface singularities characterized by the previous properties. They go under many different names: simple singularities, ADE singularities, Du Val singularities, double rational points, Gorenstein quotient singularities, Klein singularities.

TABLE 3. Simple surface singularities

\begin{tabular}{||l|r|r|r|r|}
\hline Type & Polynomial & Weights & Degree & $\mathrm{G}$ \\
\hline$A_{2 k}, k \geq 1$ & $Z_{1} Z_{2}+Z_{3}^{2 k+1}$ & $(2 k+1,2 k+1,2)$ & $4 k+2$ & $\mathrm{C}_{4 k}$ \\
\hline$A_{2 k+1}, k \geq 0$ & $Z_{1} Z_{2}+Z_{3}^{2 k+2}$ & $(k+1, k+1,1)$ & $2 k+2$ & $\mathrm{C}_{2 k+1}$ \\
\hline$D_{n}, n \geq 4$ & $Z_{1}^{2}+Z_{2} Z_{3}^{2}+Z_{2}^{n-1}$ & $(n-1,2, n-2)$ & $2 n-2$ & $D_{4 n-4}$ \\
\hline$E_{6}$ & $Z_{1}^{2}+Z_{2}^{3}+Z_{3}^{4}$ & $(6,4,3)$ & 12 & $T_{24}$ \\
\hline$E_{7}$ & $Z_{1}^{2}+Z_{2}^{3}+Z_{2} Z_{3}^{3}$ & $(9,6,4)$ & 18 & $O_{48}$ \\
\hline$E_{8}$ & $Z_{1}^{2}+Z_{2}^{3}+Z_{3}^{5}$ & $(15,10,6)$ & 30 & $I_{120}$ \\
\hline
\end{tabular}

The following result follows immediately from Brieskorn's and Tjurina's construction of simultaneous resolution of simple surface singularities [15], [92], [111].

Theorem 8.8. The Milnor lattice of a simple surface singularity is a finite root lattice of the type indicated in the first column of Table 3 with quadratic form multiplied by -1 . The monodromy group is the corresponding finite real reflection group.

It is known that the incidence graph of the irreducible components of a minimal resolution of singularities is the Coxeter graph of the corresponding type. This was first observed by P. Du Val [37].

Consider the monodromy group of a simple surface singularity $\Gamma$ of $(X, 0)$ as the image of the monodromy map (Remark 8.3). For each Coxeter system $(W, S)$ with Coxeter matrix $\left(m_{s s^{\prime}}\right)$ one defines the associated Artin-Brieskorn braid group $B_{W}$ by presentation

$$
B_{W}=\{g_{s}, s \in S: \underbrace{\left(g_{s} g_{s^{\prime}} g_{s}\right) \cdots\left(g_{s} g_{s^{\prime}} g_{s}\right)}_{m\left(s, s^{\prime}\right)-2}=\underbrace{\left(g_{s^{\prime}} g_{s} g_{s^{\prime}}\right) \cdots\left(g_{s^{\prime}} g_{s} g_{s^{\prime}}\right)}_{m\left(s, s^{\prime}\right)-2}\} .
$$

If we impose additional relations $g_{s}^{2}=1, s \in S$, we get the definition of $(W, S)$. This defines an extension of groups

$$
1 \rightarrow \tilde{B}_{W} \rightarrow B_{W} \rightarrow W \rightarrow 1,
$$

where $\tilde{B}_{W}$ is the normal subgroup of $B_{W}$ generated by conjugates of $g_{s}^{2}$. 
Brieskorn also proves the following.

Theorem 8.9. Let $\Delta \subset \mathbb{C}^{\mu}$ be the discriminant of a simple surface singularity. Then $\pi_{1}\left(\mathbb{C}^{\mu} \backslash \Delta, u_{0}\right)$ is isomorphic to the braid group $B_{\Gamma}$ of the monodromy group $\Gamma$. The regular covering $U \rightarrow \Delta \subset \mathbb{C}^{\mu}$ corresponding to the normal subgroup $\tilde{B}_{\Gamma}$ can be $\Gamma$-equivariantly extended to the covering $V \rightarrow V / \Gamma \cong \mathbb{C}^{\mu}$, where $V$ is a complex vector space of dimension $\mu$ on which $\Gamma$ acts as a reflection group. The pre-image of $\Delta$ in $V$ is the union of reflection hyperplanes.

Example 8.10. The following properties are equivalent.

- $\mu_{+}=0, \mu_{0}>0$;

- $\mu_{+}=0, \mu_{0}=2$;

- the exceptional curve of a minimal resolution is a nonsingular elliptic curve;

- $(V, 0)$ can be represented by the zero level of one of the polynomials given in Table 4.

These singularities are called simple elliptic singularities.

TABLE 4. Simple elliptic surface singularities

\begin{tabular}{||l|r|r|r|}
\hline Type & Polynomial & Weights & Degree \\
\hline$P_{8}$ & $z_{1}^{3}+z_{2}^{3}+z_{3}^{4}+\lambda z_{1} z_{2} z_{3}$ & $(1,1,1)$ & 3 \\
\hline$X_{9}$ & $z_{1}^{2}+z_{2}^{4}+z_{3}^{4}+\lambda z_{1} z_{2} z_{3}$ & $(2,1,1)$ & 4 \\
\hline$J_{10}$ & $z_{1}^{2}+z_{2}^{3}+z_{3}^{6}+\lambda z_{1} z_{2} z_{3}$ & $(3,2,1)$ & 6 \\
\hline
\end{tabular}

Here the subscript is equal to $\mu$.

Theorem 8.11 (A. Gabrielov [45]). Let $M$ be the Milnor lattice of a simple elliptic singularity. Then $M^{\perp}$ is of rank 2 and $M / M^{\perp}$ is isomorphic to the root lattice of type $E_{\mu-2}$. The image $\bar{G}$ of the monodromy group $\Gamma$ in $\left(M / M^{\perp}\right)$ is the finite reflection group of type $E_{\mu-2}$. The monodromy group is isomorphic to the semidirect product $\left(M^{\perp} \otimes M / M^{\perp}\right) \rtimes W\left(E_{\mu-2}\right)$ and can be naturally identified with an affine complex crystallographic reflection group with linear part $W\left(E_{\mu-2}\right)$.

There is a generalization of Theorem 8.9 to the case of simple elliptic singularities due to E. Looijenga [74] and [91]. It involves affine crystallographic reflection groups and uses Theorem 3.3 .

Example 8.12. The following properties are equivalent:

- $\mu_{+}=1$;

- $\mu_{+}=1, \mu_{0}=1$;

- $V$ can be given by equation $P\left(z_{1}, z_{2}, z_{3}\right)=0$, where

$$
P=z_{1}^{a}+z_{2}^{b}+z_{3}^{c}+\lambda z_{1} z_{2} z_{3}, \quad \frac{1}{a}+\frac{1}{b}+\frac{1}{c}<1, \lambda \neq 0 .
$$

These singularities are called hyperbolic unimodal singularities.

Theorem 8.13 (A. Gabrielov [45]). The Milnor lattice $M$ of a hyperbolic singularity is isomorphic to the lattice

$$
E_{p, q, r}(-1) \perp\langle 0\rangle .
$$

The monodromy group is the semi-direct product $\mathbb{Z}^{\mu} \rtimes W(p, q, r)$. Its image in $\mathrm{O}\left(M / M^{\perp}\right)$ is the reflection group $W(p, q, r)$. 
The previous classes of isolated surface singularities are characterized by the condition $\mu_{+} \leq 1$. If $\mu_{+} \geq 2$, the monodromy group is always of finite index in $\mathrm{O}(M)$ (see [40], [41]). Together with the previous theorems this implies that the monodromy group is always of finite index in $\mathrm{O}(M)$ except in the case of hyperbolic unimodal singularities with $(p, q, r)=(2,3,7),(2,4,5),(3,3,4)$ (see Example 4.11).

There is a generalization of Brieskorn's theorem 8.9 to the case of hyperbolic singularities due to E. Looijenga [74], [75].

\section{Symmetries of Singularities}

9.1. Eigen-monodromy groups. Suppose we have a holomorphic map $f: X \rightarrow$ $S$ as in section 8.1. Suppose also that a finite group $G$ acts on all fibres of the map in a compatible way. This means that there is an action of $G$ on $X$ which leaves fibres invariant. Then the cohomology groups $H_{c}^{n}\left(X_{s}, \mathbb{C}\right)$ become representation spaces for $G$, and we can decompose them into irreducible components

$$
H_{c}^{n}\left(X_{s}, \mathbb{C}\right)=\bigoplus_{\chi \in \operatorname{Irr}(G)} H_{c}^{n}\left(X_{s}, \mathbb{C}\right)_{\chi}
$$

One checks that the monodromy map decomposes too and defines the $\chi$-monodromy map

$$
\rho_{s_{0}}^{\chi}: \pi_{1}\left(S ; s_{0}\right) \rightarrow \mathrm{GL}\left(H_{c}^{n}\left(X_{s}, \mathbb{C}\right)_{\chi}\right) .
$$

Let $E$ be a real vector space equipped with a bilinear form $(v, w)$, symmetric or skew-symmetric. Let $E_{\mathbb{C}}$ be its complexification with the conjugacy map $v \mapsto \bar{v}$. We extend the bilinear form on $E$ to $E_{\mathbb{C}}$ by linearity. It is easy to see that it satisfies $(\bar{x}, \bar{y})=\overline{(x, y)}$. Next we equip $E_{\mathbb{C}}$ with a hermitian form defined by

$$
\langle x, y\rangle= \begin{cases}(x, \bar{y}), & \text { if }(x, y) \text { is symmetric, } \\ i(x, \bar{y}) & \text { otherwise. }\end{cases}
$$

We apply this to $E_{\mathbb{C}}=H_{c}^{n}\left(X_{s}, \mathbb{C}\right)$ with the bilinear map defined by the cupproduct. The $\chi$-monodromy map leaves the corresponding hermitian form invariant and defines a homomorphism

$$
\rho_{s_{0}}: \pi_{1}\left(S ; s_{0}\right) \rightarrow \mathrm{U}\left(H_{c}^{n}\left(X_{s}, \mathbb{C}\right)_{\chi}\right) .
$$

We are interested in examples when the image of this homomorphism is a complex reflection group.

9.2. Symmetries of singularities. Assume that the germ of an isolated hypersurface singularity $(X, 0)$ can be represented by a polynomial $f$ which is invariant with respect to some finite subgroup $G$ of $\operatorname{GL}(n+1, \mathbb{C})$. One can define the notion of a $G$-equivariant deformation of $(X, 0)$ and show that a semi-universal $G$-equivariant deformation of $(X, 0)$ can be given by the germ of the map

$$
\Phi_{G}:\left(\mathbb{C}^{n+\tau^{\prime}}, 0\right) \rightarrow\left(\mathbb{C}^{\tau^{\prime}}, 0\right),(z, u) \rightarrow\left(f(z)+\sum_{i=1}^{\tau^{\prime}} u_{i} g_{i}, u_{1}, \ldots, u_{\tau^{\prime}-1}\right)
$$

where $\left(g_{1}, \ldots, g_{\tau^{\prime}}\right)$ is a basis of the subspace $J_{f=0}^{G, \chi}$ of relative invariants of the algebra (8.6). In the case when $G$ is cyclic, we can choose $g_{i}$ 's to be monomials. The equivariant discriminant $\Delta_{G}$ is defined the same as in the case of the trivial action. Now we define the $G$-equivariant monodromy group $\Gamma_{G}$ of $\left(X, x_{0}\right)$ following the definition in the case $G=\{1\}$. The group $G$ acts obviously on the Milnor fibre 
of $f$ and hence on the Milnor lattice $M$, giving it a structure of a $\mathbb{Z}[G]$-module. Since

$$
\mathbb{C}^{\tau^{\prime}} \backslash \Delta_{G} \subset \mathbb{C}^{\tau} \backslash \Delta
$$

we can choose a point $s_{0} \in \mathbb{C}^{\tau^{\prime}} \backslash \Delta_{G}$ to define a homomorphism

$$
i_{s_{0}}: \pi_{1}\left(\mathbb{C}^{\tau^{\prime}} \backslash \Delta_{G} ; s_{0}\right) \rightarrow \pi_{1}\left(\mathbb{C}^{\tau} \backslash \Delta ; s_{0}\right) .
$$

This homomorphism induces a natural injective homomorphism of the monodromy groups $i_{*}: \Gamma_{G} \rightarrow \Gamma$. This allows us to identify $\Gamma_{G}$ with a subgroup of $\Gamma$.

Proposition 9.1 (P. Slodowy [107]). Let $\Gamma$ be the monodromy group of $\left(X, x_{0}\right)$ and $\Gamma_{G}$ be the $G$-equivariant monodromy group. Then

$$
\Gamma_{G}=\Gamma \cap A u t_{\mathbb{Z}[G]}(M) .
$$

Consider the natural action of $G$ on the jacobian algebra (8.2) via its action on the partial derivatives of the function $f$.

Theorem 9.2 (C.T.C. Wall [121]). Assume $\left(X, x_{0}\right)$ is an isolated hypersurface singularity defined by a holomorphic function $f: \mathbb{C}^{n+1} \rightarrow \mathbb{C}$. Let $J_{f}$ be its jacobian algebra. There is an isomorphism of $G$-modules,

$$
M_{\mathbb{C}} \cong J_{f} \otimes \operatorname{det}_{G},
$$

where $\operatorname{det}_{G}$ is the one-dimensional representation of $G$ given by the determinant.

Example 9.3. Let $f\left(z_{1}, z_{2}, z_{3}\right)=z_{1} z_{2}+z_{3}^{2 k}$ be a simple surface singularity of type $A_{2 k-1}$. Consider the action of the group $G=\mathbb{Z} / 2 \mathbb{Z}$ by $\left(z_{1}, z_{2}, z_{3}\right) \mapsto\left(z_{1}, z_{2},-z_{3}\right)$. We take $1, z_{3}, \ldots, z_{3}^{2 k-2}$ to be a basis of the jacobian algebra. Thus we have $k$ invariant monomials $1, z_{3}^{2}, \ldots, z_{3}^{2 k-2}$ and $k-1$ anti-invariant monomials $z_{3}, \ldots, z_{3}^{2 k-3}$. It follows from Theorem 9.2 that $M_{\mathbb{C}}$ is the direct sum of the $k-1$-dimensional invariant part $M_{+}$and the $k$-dimensional anti-invariant part $M_{-}$. We have

$$
\mathrm{O}(M)=W\left(A_{2 k-1}\right) \rtimes(\tau),
$$

where $\tau$ is the nontrivial symmetry of the Coxeter diagram of type $A_{2 k-1}$. In fact it is easy to see that the semi-direct product is the direct product. Let $\alpha_{1}, \ldots, \alpha_{2 k-1}$ be the fundamental root vectors. We have $\tau\left(\alpha_{i}\right)=\alpha_{2 k-i}$; hence $\operatorname{dim} M^{\tau}=k$. This shows that the image $\sigma$ of the generator of $G$ in $\mathrm{O}(M)$ is not equal to $\tau$. In fact, it must belong to $W\left(A_{2 k-1}\right)$. To see this we use that all involutions in $W(M) \cong \Sigma_{2 k}$ are the products of at most $k$ transpositions; hence their fixed subspaces are of dimension $\leq k-1$. The group $\mathrm{O}(M)$ is the product of $W(M)$ and \pm 1 ; thus all involutions in $\mathrm{O}(M) \backslash W(M)$ have fixed subspaces of dimension $\leq k-1$. The conjugacy class of $\sigma \in W\left(A_{2 k-1}\right) \cong \Sigma_{2 k}$ is determined by the number $r$ of disjoint transpositions in which it decomposes. We have $\operatorname{dim} M^{\sigma}=2 k-r-1$. Thus $\sigma$ is conjugate to the product of $k$ disjoint transpositions. It follows from the model of the lattice $A_{2 k-1}$ given in (2.1) that $\sigma$ is conjugate to the transformation $\alpha_{i} \mapsto$ $-\alpha_{2 k-i}$. Hence the sublattice $M_{-}$is generated by $\beta_{i}=\alpha_{i}+\alpha_{2 k-i}, i=1, \ldots, k-1$, and $\beta_{k}=\alpha_{k}$. We have

$$
\left(\beta_{i}, \beta_{j}\right)= \begin{cases}-4 & \text { if } i=j \neq k \\ -2 & \text { if } i=j=k \\ 2 & \text { if }|i-j|=1 \\ 0 & \text { if }|i-j|>1\end{cases}
$$


Comparing this with Example 4.1 we find that $M=N(2)$, where $N$ is the lattice defining an integral structure for the reflection group of type $B_{k}$. In other words, the reflections $r_{\beta_{i}}$ generate the Weyl group of type $B_{k}$.

Similar construction for a symmetry of order 2 of singular points of types $D_{k+1}$, $n \geq 4$ (resp. $E_{6}$, resp. $D_{4}$ ), leads to the reflection groups of type $B_{k}$ (resp. $F_{4}$, resp. $\left.G_{2}=I_{2}(6)\right)$. The Milnor lattices obtained as the invariant parts of the Milnor lattice of type $A_{2 k-1}$ and $D_{k+1}$ define the same reflection groups, but their lattices are similar but not isomorphic. Figure 14 shows how the Coxeter diagrams of types $B_{k}, F_{4}, G_{2}$ can be obtained from those of types $A, D, E$.
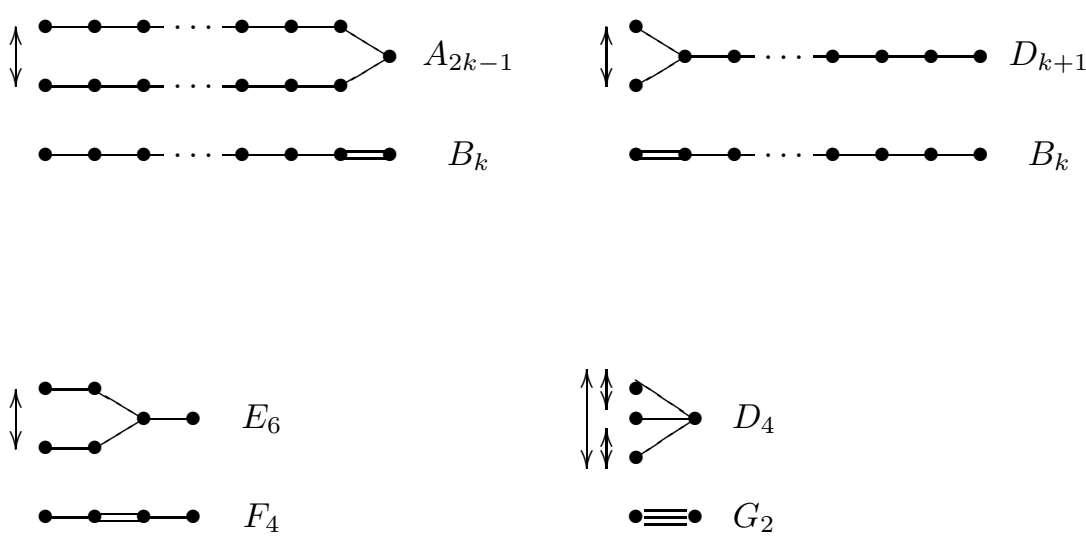

FIGURE 14

Remark 9.4. The appearance of the Dynkin diagrams of type $B_{n}, F_{4}, G_{2}$ in the theory of simple singularities was first noticed by P. Slodowy [106], and from a different but equivalent perspective in the work of Arnol'd on critical points on manifolds with boundary [6], [7]. In the theory of simple surface singularities over nonalgebraically closed field, they appear in [73].

Example 9.5. Let $(X, 0)$ be a simple surface singularity of type $E_{6}$ given by equation $z_{1}^{2}+z_{2}^{3}+z_{3}^{4}=0$. Consider the group $G$ generated by the symmetry $g$ of order 3 given by $\left(z_{1}, z_{2}, z_{3}\right) \mapsto\left(z_{1}, \eta_{3} z_{2}, z_{3}\right)$, where $\eta_{3}=e^{2 \pi i / 3}$. A monomial basis of the jacobian algebra is $\left(1, z_{2}, z_{3}, z_{3}^{2}, z_{2} z_{3}, z_{2} z_{3}^{2}\right)$. By Theorem 9.2 , we have

$$
M_{\mathbb{C}}=\left(M_{\mathbb{C}}\right)_{\chi} \oplus\left(M_{\mathbb{C}}\right)_{\bar{\chi}}
$$

where $\chi(g)(x)=\eta_{3} x$. The characteristic polynomial of $g$ in $M_{\mathbb{C}}$ is equal to $\left(t^{2}+\right.$ $t+1)^{3}$. We have

$$
\mathrm{O}(M)=W\left(E_{6}\right) \rtimes(\mathbb{Z} / 2 \mathbb{Z}),
$$

where the extra automorphism is defined by the symmetry of the Coxeter diagram. Since $g$ is of order 3 , its image $w$ in $\mathrm{O}\left(E_{6}\right)$ belongs to $W\left(E_{6}\right)$. The classification of elements of order 3 in the Weyl group $W\left(E_{6}\right)$ shows the conjugacy class of $w$ corresponds to the primitive embedding of lattice $A_{2}^{3} \hookrightarrow E_{6}$ so that $w$ acts as the product $c_{1} c_{2} c_{3}$ of the Coxeter elements in each copy of $A_{2}$. It is known that the centralizer of $w$ is a maximal subgroup of $W\left(E_{6}\right)$ of order $648 .{ }^{13}$ This group is

\footnotetext{
${ }^{13}$ Not to be confused with another maximal subgroup of $W\left(E_{6}\right)$ of the same order which is realized as the stabilizer subgroup of the sublattice $A_{2}^{3}$.
} 
isomorphic to the unitary complex reflection group $L_{3}$ (No. 25 in the list), and $\left(M_{\mathbb{C}}\right)_{\chi}$ is its three-dimensional reflection representation.

This and other examples of appearance of finite unitary complex reflection groups as the $G$-equivariant monodromy groups were first constructed by V. Goryunov [49], $[48]$.

Affine complex crystallographic reflection groups can also be realized as $G$ equivariant monodromy groups. We give only one example, referring for more to $[51],[50]$.

Example 9.6. Consider a simple elliptic singularity of type $J_{10}$ with parameter $\lambda=0$. Its equation is given in Table 4 . Consider the symmetry of order 3 defined by an automorphism $g:\left(z_{1}, z_{2}, z_{3}\right) \mapsto\left(z_{1}, \zeta_{3} z_{2}, z_{3}\right)$. A monomial basis of the jacobian algebra is $\left(1, z_{2}, z_{3}, z_{3}^{2}, z_{3}^{4}, z_{2} z_{3}, z_{2} z_{3}^{2}, z_{2} z_{3}^{4}\right)$. We have 5 invariant monomials: $1, z_{3}, z_{3}^{2}, z_{3}^{3}, z_{3}^{4}$. Applying Theorem 9.2 we obtain that $M_{\mathbb{C}}=\left(M_{\mathbb{C}}\right)_{\chi} \oplus\left(M_{\mathbb{C}}\right)_{\bar{\chi}}$, both summands of dimension 5. The characteristic polynomial of $g$ is equal to $\left(1+t+t^{2}\right)^{5}$. Obviously, $g$ leaves $M^{\perp}$ invariant, and the image $w=\bar{g}$ of $g$ in $\mathrm{O}(M / M)=\mathrm{O}\left(E_{8}\right)=W\left(E_{8}\right)$ has characteristic polynomial $\left(1+t+t^{2}\right)^{4}$. It follows from the classification of conjugacy classes in $W\left(E_{8}\right)$ that $w$ is the product of the Coxeter elements in the sublattice $A_{2}^{4}$ of $E_{8}$. Its centralizer is a subgroup of index 2 in the wreath product $\Sigma_{3}^{4} \succ \Sigma_{4}$ of order 31104 . This group is a finite complex reflection group $L_{4}$ (No. 32 in the list). The centralizer of $g$ is the unique complex crystallographic group in affine space of dimension 5 with linear part $L_{4}$.

\section{Complex Ball Quotients}

10.1. Hypergeometric integrals. Let $S$ be an ordered set of $n+3$ distinct points $z_{1}, \ldots, z_{n+3}$ in $\mathbb{P}^{1}(\mathbb{C})$. We assume that $\left(z_{n+1}, z_{n+2}, z_{n+3}\right)=(0,1, \infty)$. Let $U=\mathbb{P}^{1} \backslash$ $S$ and $\gamma_{1}, \ldots, \gamma_{n+3}$ be the standard generators of $\pi_{1}\left(U ; u_{0}\right)$ satisfying the relation $\gamma_{1} \cdots \gamma_{n+3}=1$. We have a canonical surjection of the fundamental group of $U$ to the group $A=(\mathbb{Z} / d \mathbb{Z})^{n+3} / \Delta(\mathbb{Z} / d \mathbb{Z})$ which defines an étale covering $V \rightarrow U$ with the Galois group $A$. The open Riemann surface $V$ extends $A$-equivariantly to a compact Riemann surface $X(z)$ with quotient $X(z) / A$ isomorphic to $\mathbb{P}^{1}(\mathbb{C})$.

Let $\boldsymbol{\mu}=\left(m_{1} / d, \ldots, m_{n+3} / d\right)$ be a collection of rational numbers in the interval $(0,1)$ satisfying

$$
\frac{1}{d} \sum_{i=1}^{n+3} m_{i}=k \in \mathbb{Z} .
$$

They define a surjective homomorphism

$$
\chi: A \rightarrow \mathbb{C}^{*}, \quad \bar{\gamma}_{i} \mapsto e^{2 \pi \sqrt{-1} m_{i} / d},
$$

where $\bar{\gamma}_{i}$ is the image of $\gamma_{i}$ in $A$.

The following computation can be found in [28] (see also [30]).

Lemma 10.1. Let $H^{1}(X(z), \mathbb{C})_{\chi}$ be the $\chi$-eigensubspace of the natural representation of the Galois group $A$ on $H^{1}(X(z), \mathbb{C})$. Then

$$
\operatorname{dim} H^{1}(X(z), \mathbb{C})_{\chi}=n+1 .
$$

Let $\Omega(X(z))$ be the space of holomorphic 1-forms on $X(z)$ and $\Omega(X(z))_{\chi}$ be the $\chi$-eigensubspace of $A$ in its natural action on the space $\Omega(X(z))$. Then

$$
\operatorname{dim} \Omega(X(z))_{\chi}=k-1
$$


where $k$ is defined in (10.1).

Recall that $H^{1}(X(z), \mathbb{C})=\Omega(X(z)) \oplus \bar{\Omega}(X(z))$, so we get

$$
H^{1}(X(z), \mathbb{C})_{\chi}=\Omega(X(z))_{\chi} \oplus \bar{\Omega}(X(z))_{\chi},
$$

and we can consider the hermitian form in $H^{1}(X(z), \mathbb{C})_{\chi}$ induced by the skewsymmetric cup-product

$$
H^{1}(X(z), \mathbb{C}) \times H^{1}(X(z), \mathbb{C}) \rightarrow H^{2}(X(z), \mathbb{C}) \cong \mathbb{C} .
$$

The signature of the hermitiain form is equal to $(k-1, n-k+2)$.

Now let us start to vary the points $z_{1}, \ldots, z_{n}$ in $\mathbb{P}^{1}(\mathbb{C})$ but keep them distinct and not equal to 0,1 or $\infty$. Let $\mathcal{U} \subset \mathbb{P}^{1}(\mathbb{C})^{n}$ be the corresponding set of parameters. Its complement in $\left(\mathbb{P}^{1}(\mathbb{C})^{n}\right)$ consists of $N=\left(\begin{array}{l}n \\ 2\end{array}\right)+3 n$ hyperplanes $H_{i j}: z_{i}-z_{j}=0$ and $H_{i}(0): z_{i}=0, H_{i}(1): z_{i}=1, H_{i}(\infty): z_{i}=\infty$. Fix a point $z^{(0)} \in \mathcal{U}$. For each of these hyperplanes $H$ consider a path which starts at $z^{(0)}$, goes to a point on a small circle normal bundle of an open subset of $H$, goes along the circle, and then returns to the starting point. The homotopy classes $s_{1}, \ldots, s_{N}$ of these paths generate $\pi_{1}\left(\mathcal{U} ; z^{(0)}\right)$.

It is not difficult to construct a fibration over $\mathcal{U}$ whose fibres are the curves $X(z)$. This defines a local coefficient system $\mathcal{H}(\chi)$ over $\mathcal{U}$ whose fibres are the spaces $H^{1}(X(z), \mathbb{C})_{\chi}$ and the monodromy map

$$
\pi_{1}\left(\mathcal{U} ; z^{(0)} \rightarrow \mathrm{U}\left(H^{1}\left(X\left(z^{(0)}\right), \mathbb{C}\right)_{\chi}\right) .\right.
$$

Denote the monodromy group by $\Gamma(\boldsymbol{\mu})$.

The important case for us is when $|\boldsymbol{\mu}|=2$. In this case the signature is $(1, n)$, and we can consider the image of the monodromy group $\Gamma(\boldsymbol{\mu})$ in $\mathbb{P} U(1, n))$ which acts in the complex hyperbolic space $H_{\mathbb{C}}^{n}$.

Here is the main theorem from [28], [80].

Theorem 10.2. The image of each generator $s_{i}$ of $\pi_{1}\left(\mathcal{U} ; z^{(0)}\right)$ in $\Gamma(\boldsymbol{\mu})$ acts as a complex reflection in the hyperbolic space $H_{\mathbb{C}}^{n}$. The group $\Gamma(\boldsymbol{\mu})$ is a crystallographic reflection group in $H_{\mathbb{C}}^{n}$ if and only if one of the following conditions is satisfied:

- $\left(1-\frac{m_{i}}{d}-\frac{m_{j}}{d}\right)^{-1} \in \mathbb{Z}, i \neq j, m_{i}+m_{j}<1$;

- $2\left(1-\frac{m_{i}}{d}-\frac{m_{j}}{d}\right)^{-1} \in \mathbb{Z}$, if $m_{i}=m_{j}, i \neq j$.

All possible $\boldsymbol{\mu}$ satisfying the conditions from the theorem can be enumerated. We have 59 cases if $n=2,20$ cases if $n=3,10$ cases if $n=4,6$ cases when $n=5$, 3 cases if $n=6,2$ cases when $n=7$, and 1 case if $n=8$ or $n=9$. There are several cases when the monodromy group is cocompact. It does not happen in dimension $n>7$.

The orbit spaces $H_{\mathbb{C}}^{n} / \Gamma(\boldsymbol{\mu})$ of finite volume have a moduli theoretical interpretation. It is isomorphic to the geometric invariant theory quotient $\left(\mathbb{P}^{1}\right)^{n+3} / / \operatorname{SL}(2)$ with respect to an appropriate choice of linearization of the action.

We refer to Mostow's survey paper [81], where he explains a relation between the monodromy groups $\Gamma(\boldsymbol{\mu})$ and the monodromy groups of hypergeometric integrals.

10.2. Moduli space of Del Pezzo surfaces as complex ball quotients. In the last section we will discuss some recent work on complex ball uniformization of some moduli spaces in algebraic geometry.

It is well-known that a nonsingular cubic curve in the projective plane is isomorphic as a complex manifold to a complex torus $\mathbb{C} / \mathbb{Z}+\mathbb{Z} \tau$, where $\tau$ belongs to the 
upper-half plane $\mathcal{H}=\{a+b i \in \mathbb{C}: b>0\}$. Two such tori are isomorphic if and only if the corresponding $\tau$ 's belong to the same orbit of the group $\Gamma=\operatorname{SL}(2, \mathbb{Z})$ which acts on the $\mathcal{H}$ by Möbius transformations $z \mapsto(a z+b) /(c z+d)$. This result implies that the moduli space of plane cubic curves is isomorphic to the orbit space $\mathcal{H} / \Gamma$. Of course, the upper-half plane is a model of the one-dimensional complex hyperbolic space $H_{\mathbb{C}}^{1}$ and the group $\Gamma$ acts as a crystallographic reflection group.

In a beautiful paper of D. Allcock, J. Carlson and D. Toledo [2], the complex ball uniformization of the moduli space of plane cubics is generalized to the case of the moduli space of cubic surfaces in $\mathbb{P}^{3}(\mathbb{C})$. It has been known since the last century that the linear space $V$ of homogeneous forms of degree 3 in 4 variables admits a natural action of the group SL(4) such that the algebra of invariant polynomial functions on $V$ of degree divisible by 8 is freely generated by invariants $I_{8}, I_{16}, I_{24}, I_{32}, I_{40}$ of degrees indicated by the subscript. This can be interpreted as saying that the moduli space of nonsingular cubic surfaces admits a compactification isomorphic to the weighted projective space $\mathbb{P}(1,2,3,4,5)$. Let $F\left(T_{0}, T_{1}, T_{2}, T_{3}\right)=0$ be an equation of a nonsingular cubic surface $S$. Adding the cube of a new variable $T_{4}$, we obtain an equation

$$
F\left(T_{0}, T_{1}, T_{2}, T_{3}\right)+T_{4}^{3}=0
$$

of a nonsingular cubic hypersurface $X$ in $\mathbb{P}^{4}(\mathbb{C})$. There is a construction of an abelian variety of dimension 10 attached to $X$ (the intermediate jacobian) $\operatorname{Jac}(X)$. The variety $X$ admits an obvious automorphism of order 3 defined by multiplying the last coordinate by a third root of unity. This makes $\operatorname{Jac}(X)$ a principally polarized abelian variety of dimension 10 with complex multiplication of certain type. ${ }^{14}$ The moduli space of such varieties is known to be isomorphic to a quotient of a 4-dimensional complex ball by a certain discrete subgroup $\Gamma$. It is proven in [2] that the group $\Gamma$ is a hyperbolic complex crystallographic reflection group and the quotient $H_{\mathbb{C}}^{4} / \Gamma$ is isomorphic to the moduli space of cubic surfaces with at most ordinary double points as singularities. By adding one point one obtains a compactification of the moduli space isomorphic to the weighted projective space $\mathbb{P}(1,2,3,4,5)$.

The geometric interpretation of reflection hyperplanes is also very nice; they form one orbit representing singular surfaces. The group $\Gamma$ contains a normal subgroup $\Gamma^{\prime}$ with quotient isomorphic to the Weyl group $W\left(E_{6}\right)$. The quotient subgroup $H_{\mathbb{C}}^{4} / \Gamma^{\prime}$ is the moduli space of marked nodal cubic surfaces. For a nonsingular surface a marking is a fixing of order on the set of 27 lines on the surface.

We mentioned before that some complex ball quotients appear as the moduli space of K3 surfaces which admit an action of a cyclic group $G$ with fixed structure of the sublattice $\left(S_{X}\right)^{G}$. This idea was used by S. Kondō to construct an action of a crystallographic reflection group $\Gamma$ in a complex ball $H_{\mathbb{C}}^{6}\left(\right.$ resp. $\left.H_{\mathbb{C}}^{9}\right)$ with orbit space containing the moduli space of nonsingular plane quartic curves of genus 3 (resp. moduli space of canonical curves of genus 4). ${ }^{15}$ In the first case he assigns to a plane quartic $F\left(T_{0}, T_{1}, T_{2}\right)=0$ the quartic K3-surface

$$
F\left(T_{0}, T_{1}, T_{2}\right)+T_{3}^{4}=0
$$

\footnotetext{
${ }^{14}$ This beautiful idea of assigning to a cubic surface a certain abelian variety was independently suggested by B. van Geemen and B. Hunt.

${ }^{15} \mathrm{It}$ is isomorphic to the moduli space of Del Pezzo surfaces of degree 2.
} 
with automorphism of order 4 and $S_{X}^{G} \cong U(2) \perp A_{1}(-1)^{6}$. This leads to a new example of a crystallographic reflection group in $H_{\mathbb{C}}^{6}$. It is known that a canonical curve $C$ of genus 4 is isomorphic to a complete intersection of a quadric and cubic in $\mathbb{P}^{3}(\mathbb{C})$. To each such curve Kondo assigns the K3 surface isomorphic to the triple cover of the quadric branched along the curve $C$. It has an action of a cyclic group of order 3 with $\left(S_{X}\right)^{G} \cong U \perp A_{2}(-2)$. The stabilizer of a reflection hyperplane gives a complex reflection group in $H_{\mathbb{C}}^{8}$ with quotient isomorphic to a partial compactification of the moduli space of Del Pezzo surfaces of degree 1. Independently such a construction was found in [56].

Finally, one can also re-prove the result of Allcock-Carlson-Toledo by using K3 surfaces instead of intermediate jacobians (see [32]).

Remark 10.3. All reflection groups arising in these complex ball uniformization constructions are not contained in the Deligne-Mostow list (corrected in [110]). However, some of them are commensurable ${ }^{16}$ with some groups from the list. For example, the group associated to cubic surfaces is commensurable to the group $\Gamma(\boldsymbol{\mu})$, where $d=6, m_{1}=m_{2}=1, m_{3}=\ldots=m_{7}=2$. We refer to [25] for a construction of complex reflection subgroups of finite volume which are not commensurable to the groups from the Deligne-Mostow list. No algebraic-geometrical interpretation of these groups is known so far.

\section{ACKNOWLEDGEMENTS}

This paper is dedicated to Ernest Borisovich Vinberg, one of the heroes of the theory of reflection groups. His lectures for high school children in Moscow were influential (without his knowledge) in my decision to become a mathematician.

The paper is an expanded version of my colloquium lecture at the University di Roma Terzo in May 2006. I am thankful to Alessandro Verra for giving me an opportunity to give this talk and hence to write the paper. I am very grateful to Daniel Allcock, Victor Goryunov and the referee for numerous critical comments on earlier versions of the paper.

\section{ABOUt THE AUTHOR}

Igor Dolgachev is a professor at the University of Michigan in Ann Arbor. He has held visiting positions at the University of Paris, MIT, and Harvard University; and at Research Institutes in Bonn, Kyoto, Seoul, and Warwick.

\section{REFERENCES}

1. D. Allcock, The Leech lattice and complex hyperbolic reflections, Invent. Math. J. 140 (2000), 283-31. MR1756997 (2002b:11091)

2. D. Allcock, J. Carlson, and D. Toledo, The complex hyperbolic geometry of the moduli space of cubic surfaces, J. Alg. Geom. 11 (2002), 659-724. MR1910264 (2003m:32011)

3. D. Allcock, A monstrous proposal, 9 pages, math.GR/0606043. To appear in Groups and symmetries. From the Nordic Scots to John McKay, April 27-29, 2007, CRM, Montreal.

4. D. Allcock, J. Carlson, and D. Toledo, The moduli space of cubic threefolds as a ball quotient, math.AG/0608287.

\footnotetext{
${ }^{16}$ This means that the two groups share a common subgroup of finite index.
} 
5. E. Andreev, Convex polyhedra of finite volume in Lobachevskii space (Russian), Mat. Sb. (N.S.) 83 (125) (1970), 256-260. MR0273510 (42:8388)

6. V. Arnol'd, Critical points of functions on a manifold with boundary, the simple Lie groups $B_{k}, C_{k}, F_{4}$ and sequences of evolutes, Uspkhi Mat. Nauk 33 (1978), 91-105. MR511883 (80j:58008)

7. V. Arnol'd, S. Gusein-Zade, and A. Varchenko, Singularities of differentiable maps. Vol. I. Translated from the Russian. Monographs in Mathematics, 82. Birkhäuser Boston, Inc., Boston, MA, 1985. MR777682 (86f:58018)

8. W. Barth and C. Peters, Automorphisms of Enriques surfaces, Invent. Math. 73 (1983), 383-411. MR718937 (85g:14052)

9. W. Barth, K. Hulek, C. Peters, and A. Van de Ven, Compact complex surfaces, Second edition. Ergeb. der Mathematik und ihrer Grenzgebiete, Springer-Verlag, Berlin, 2004. MR2030225 (2004m:14070)

10. E. Bedford and K. Kim, Dynamics of rational surface automorphisms: linear fractional recurrences, math.DS/0611297.

11. I. Bernstein and O. Shvarzman, Chevalley's theorem for complex crystallographic Coxeter groups (Russian), Funktsional. Anal. i Prilozhen. 12 (1978), no. 4, 79-80. MR515632 (80d:32007)

12. R. Borcherds, Automorphism groups of Lorentzian lattices, J. Algebra 111 (1987), 133-153. MR913200 (89b:20018)

13. R. Borcherds, Coxeter groups, Lorentzian lattices, and K3 surfaces, Internat. Math. Res. Notices 1998, 19, 1011-1031. MR1654763 (2000a:20088)

14. N. Bourbaki, Lie groups and Lie algebras, Chapters 4-6, Translated from the 1968 French original, Elements of Mathematics (Berlin). Springer-Verlag, Berlin, 2002. MR1890629 (2003a:17001)

15. E. Brieskorn, Die Auflösung der rationalen Singulariẗ̈en holomorpher Abbildungen, Math. Ann. 178 (1968), 255-270. MR0233819 (38:2140)

16. E. Brieskorn, Singular elements of semi-simple algebraic groups, Actes du Congrès International des Mathématiciens (Nice, 1970), Tome 2, pp. 279-284. Gauthier-Villars, Paris, 1971. MR0437798 (55:10720)

17. E. Brieskorn, Die Milnorgitter der exzeptionellen unimodularen Singularitäten, Bonner Mathematische Schriften [Bonn Mathematical Publications], 150. Universität Bonn, Mathematisches Institut, Bonn, 1983. MR733785 (85k:32014)

18. V. Bugaenko, Arithmetic crystallographic groups generated by reflections, and reflective hyperbolic lattices, in "Lie groups, their discrete subgroups, and invariant theory", pp. 33-55, Adv. Soviet Math., 8, Amer. Math. Soc., Providence, RI, 1992. MR1155663 (93g:20094)

19. C. Chevalley, Invariants of finite groups generated by reflections, Amer. J. Math. 77 (1955), 778-782. MR0072877 (17:345d)

20. A. Coble, The ten nodes of the rational sextic and of the Cayley symmetroid, Amer J. Math. 41 (1919), 243-265. MR1506391

21. A. Coble, Algebraic geometry and theta functions (reprint of the 1929 edition), A. M. S. Coll. Publ., v. 10. A. M. S., Providence, RI, 1982. MR733252 (84m:14001)

22. J. Conway, The automorphism group of the 26-dimensional even unimodular Lorentzian lattice, J. Algebra 80 (1983), 159-163. MR690711 (85k:11030)

23. J. H. Conway and N.J.A. Sloane, Sphere packings, lattices, and groups, Grundlehren der Mathematischen Wissenschaften, 290. Springer-Verlag, New York, 1999. MR1662447 (2000b:11077)

24. F. Cossec and I. Dolgachev, On automorphisms of nodal Enriques surfaces, Bull. Amer. Math. Soc. (N.S.) 12 (1985), 247-249. MR776478 (86f:14028)

25. W. Couwenberg, G. Heckman, and E. Looijenga, Geometric structures on the complement of a projective arrangement, Publ. Math. Inst. Hautes Études Sci. No. 101 (2005), 69-161. MR2217047

26. H.S.M. Coxeter, The pure archimedean polytopes in six and seven dimensions, Proc. Cambridge Phil. Soc. 24 (1928), 7-9.

27. H.S.M. Coxeter, Discrete groups generated by reflections, Annals of Math. 35 (1934), 588621. MR1503182 
28. P. Deligne and G. Mostow, Monodromy of hypergeometric functions and nonlattice integral monodromy, Inst. Hautes Études Sci. Publ. Math. 63 (1986), 5-89. MR849651 (88a:22023a)

29. I. Dolgachev, Integral quadratic forms: applications to algebraic geometry (after V. Nikulin), Bourbaki seminar, Vol. 1982/83, 251-278, Astérisque, 105-106, Soc. Math. France, Paris, 1983. MR728992 (85f:14036)

30. I. Dolgachev, On automorphisms of Enriques surfaces, Invent. Math. 76 (1984), 163-177. MR739632 (85j:14076)

31. I. Dolgachev, Infinite Coxeter groups and automorphisms of algebraic surfaces, in "The Lefschetz centennial conference, Part I (Mexico City, 1984)", 91-106, Contemp. Math., 58, Amer. Math. Soc., Providence, RI, 1986. MR860406 (87j:14068)

32. I. Dolgachev, B. van Geemen, and S. Kondō, A complex ball uniformization of the moduli space of cubic surfaces via periods of K3 surfaces, J. Reine Angew. Math. 588 (2005), 99-148. MR2196731 (2006h:14051)

33. I. Dolgachev and V. Iskovskikh, Finite subgroups of the plane Cremona group, math.AG/06510595.

34. I. Dolgachev and J. Keum, Birational automorphisms of quartic Hessian surfaces, Trans. Amer. Math. Soc. 354 (2002), 3031-3057. MR1897389 (2003c:14045)

35. I. Dolgachev and S. Kondō, A supersingular K3 surface in characteristic 2 and the Leech lattice, Int. Math. Res. Not. 2003 (2003), 1-23. MR1935564 (2003i:14051)

36. I. Dolgachev and D. Ortland, Point sets in projective spaces and theta functions, Astérisque No. 165 (1988), 210 pp. (1989). MR1007155 (90i:14009)

37. P. Du Val, On singularities which do not affect the conditions of adjunction, Proc. Cambridge Phil. Society 30 (1934), 434-465.

38. P. Du Val, On the Kantor group of a set of points in a plane, Proc. London Math. Soc. 42 (1936), 18-51.

39. P. Du Val, Crystallography and Cremona transformations, in "The geometric vein", pp. 191201, Springer, New York-Berlin, 1981. MR661778 (84h:52013)

40. W. Ebeling, On the monodromy groups of singularities, Singularities, Part 1 (Arcata, Calif., 1981), 327-336, Proc. Sympos. Pure Math., 40, Amer. Math. Soc., Providence, RI, 1983. MR713071 (85d:14003)

41. W. Ebeling, The monodromy groups of isolated singularities of complete intersections, Lect. Notes in Math., vol. 1293, Springer-Verlag, Berlin, 1987. MR923114 (89d:32051)

42. F. Esselmann, Über die maximale Dimension von Lorentz-Gittern mit coendlicher Spiegelungsgruppe, J. Num. Theory 61 (1996), 103-144. MR1418323 (97g:11073)

43. G. Fano, Superficie algebriche di genere zero e bigenere uno, e loro casi particolari, Palermo Rend. 29 (1910), 98-118.

44. R. Friedman, J. Morgan, and E. Witten, Principal G-bundles over elliptic curves, Math. Res. Lett. 5 (1998), 97-118. MR1618343 (99j:14037)

45. A. Gabrielov, Dynkin diagrams of unimodal singularities (Russian), Funkcional. Anal. i Priložen. 8 (1974), no. 3, 1-6. MR0367274 (51:3516)

46. M. Gizatullin, Rational G-surfaces (Russian), Izv. Akad. Nauk SSSR Ser. Mat. 44 (1980), 110-144, 239. MR563788 (81d:14020)

47. M. Gizatullin, The decomposition, inertia and ramification groups in birational geometry, in "Algebraic geometry and its applications", eds. A. Tikhomirov and A. Tyurin, Aspects Math., E25, Vieweg, Braunschweig, 1994, pp. 39-45. MR1282018 (95d:14018)

48. V. Goryunov, Unitary reflection groups associated with singularities of functions with cyclic symmetry (Russian), Uspekhi Mat. Nauk 54 (1999), 3-24 [English transl.: Russian Math. Surveys 54 (1999), 873-893]. MR1741660 (2001b:32055)

49. V. Goryunov, Unitary reflection groups and automorphisms of simple hypersurface singularities, in "New developments in singularity theory (Cambridge, 2000)", 305-328, NATO Sci. Ser. II Math. Phys. Chem., 21, Kluwer Acad. Publ., Dordrecht, 2001. MR1849314 (2002m:32046)

50. V. Goryunov, Symmetric $X_{9}$ singularities and the complex affine reflection groups, 2005, preprint.

51. V. Goryunov and S. Man, The complex crystallographic groups and symmetries of $J_{10}, 2004$, preprint. 
52. V. Gritsenko and V. Nikulin, On classification of Lorentzian Kac-Moody algebras, Russian Math. Surveys 57:5 (2002), 921-979. MR1992083 (2004f:17034)

53. S. Gusein-Zade, Monodromy groups of isolated singularities of hypersurfaces (Russian), Uspehi Mat. Nauk 32 (1977) (194), 23-65, 263. MR0476738 (57:16295)

54. B. Harbourne, Rational surfaces with infinite automorphism group and no antipluricanonical curve, Proc. Amer. Math. Soc. 99 (1987), 409-414. MR875372 (88a:14043)

55. R. Hartshorne, Algebraic geometry, Graduate Texts in Mathematics, No. 52. Springer-Verlag, New York-Heidelberg, 1977. MR0463157 (57:3116)

56. G. Heckman and E. Looijenga, The moduli space of rational elliptic surfaces, Algebraic geometry 2000, Azumino (Hotaka), 185-248, Adv. Stud. Pure Math., 36, Math. Soc. Japan, Tokyo, 2002. MR1971517 (2004c:14068)

57. A. Hirschowitz, Symétries des surfaces rationnelles génériques, Math. Ann. 281 (1988), no. 2, 255-261. MR949832 (89k:14064)

58. J. Humphreys, Reflection groups and Coxeter groups, Cambridge Studies in Advanced Mathematics, 29. Cambridge University Press, Cambridge, 1990. MR1066460 (92h:20002)

59. B. Hunt, The geometry of some special arithmetic quotients, Lecture Notes in Mathematics, 1637. Springer-Verlag, Berlin, 1996. MR1438547 (98c:14033)

60. J. Igusa, On the structure of a certain class of Kähler varieties, Amer. J. Math. 76 (1954), 669-678. MR0063740 (16:172c)

61. A. Ivanov, A geometric characterization of the Monster, in "Groups, combinatorics \& geometry (Durham, 1990)", 46-62, London Math. Soc. Lecture Note Ser., 165, Cambridge Univ. Press, Cambridge, 1992. MR1200249 (94c:20033)

62. R. Kane, Reflection groups and invariant theory, CMS Books in Mathematics/Ouvrages de Mathématiques de la SMC, 5. Springer-Verlag, New York, 2001. MR1838580 (2002c:20061)

63. S. Kantor, Theorie der endlichen Gruppen von eindeutigen Transformationen in der Ebene, Berlin. Mayer \& Müller. 111 S. gr. $8^{\circ} .1895$.

64. J. Keum and S. Kondō, The automorphism groups of Kummer surfaces associated with the product of two elliptic curves, Trans. Amer. Math. Soc. 353 (2001), 1469-1487. MR1806732 (2001k:14075)

65. A. Khovanskii, Hyperplane sections of polyhedra, toric varieties and discrete groups in Lobachevskii space (Russian), Funktsional. Anal. i Prilozhen. 20 (1986), no. 1, 50-61, 96. MR831049 (87k:22015)

66. A. Khovanskii, Combinatorics of sections of polytopes and Coxeter groups in Lobachevsky spaces, The Coxeter Legacy, 129-157, Amer. Math. Soc., Providence, RI, 2006. MR2209026 (2006k:14091)

67. M. Koitabashi, Automorphism groups of generic rational surfaces, J. Algebra 116 (1988), no. 1, 130-142. MR944150 (89f:14045)

68. S. Kondō, Enriques surfaces with finite automorphism groups, Japan. J. Math. (N.S.) 12 (1986), 191-282. MR914299 (89c:14058)

69. S. Kondō, Automorphisms of algebraic K3 surfaces which act trivially on Picard groups, J. Math. Soc. Japan 44 (1992), 75-98. MR1139659 (93e:14046)

70. S. Kondō, The automorphism group of a generic Jacobian Kummer surface, J. Algebraic Geom. 7 (1998), 589-609. MR1618132 (99i:14043)

71. S. Kondō, A complex hyperbolic structure for the moduli space of curves of genus three, J. Reine Angew. Math. 525 (2000), 219-232. MR1780433 (2001j:14039)

72. S. Kondō, The moduli space of curves of genus 4 and Deligne-Mostow's complex reflection groups, Algebraic geometry 2000, Azumino (Hotaka), 383-400, Adv. Stud. Pure Math., 36, Math. Soc. Japan, Tokyo, 2002. MR1971521 (2004h:14033)

73. J. Lipman, Rational singularities, with applications to algebraic surfaces and unique factorization, Inst. Hautes Études Sci. Publ. Math. No. 36 (1969) 195-279. MR0276239 (43:1986)

74. E. Looijenga, Homogeneous spaces associated to certain semi-universal deformations, Proceedings of the International Congress of Mathematicians (Helsinki, 1978), pp. 529-536, Acad. Sci. Fennica, Helsinki, 1980. MR562651 (81j:14006)

75. E. Looijenga, Invariant theory for generalized root systems, Invent. Math. 61 (1980), no. 1, 1-32. MR587331 (82f:17011)

76. E. Looijenga, Isolated singular points on complete intersections, London Math. Soc. Lecture Note Series, 77. Cambridge Univ. Press, Cambridge, 1984. MR747303 (86a:32021) 
77. E. Looijenga and R. Swiersa, The period map for cubic threefolds, math.AG/0608279.

78. C. McMullen, Dynamics of blowups of the projective plane, Publ. Math. IHES (to appear).

79. J. Milnor, Singular points of complex hypersurfaces, Annals of Mathematics Studies, No. 61, Princeton University Press, Princeton, NJ; University of Tokyo Press, Tokyo, 1968. MR0239612 (39:969)

80. G. Mostow, Generalized Picard lattices arising from half-integral conditions, Inst. Hautes Études Sci. Publ. Math. 63 (1986), 91-106. MR849652 (88a:22023b)

81. G. Mostow, Braids, hypergeometric functions, and lattices, Bull. Amer. Math. Soc. (N.S.) 16 (1987), no. 2, 225-246. MR876959 (88e:22017)

82. S. Mukai, Geometric realization of T-shaped root systems and counterexamples to Hilbert's fourteenth problem, in "Algebraic transformation groups and algebraic varieties", 123-129, Encyclopaedia Math. Sci., 132, Springer, Berlin, 2004. MR2090672 (2005h:13008)

83. Y. Namikawa, Periods of Enriques surfaces, Math. Ann. 270 (1985), 201-222. MR771979 $(86 \mathrm{j}: 14035)$

84. V. Nikulin, Quotient-groups of groups of automorphisms of hyperbolic forms by subgroups generated by 2-reflections. Algebro-geometric applications. Current problems in mathematics, Vol. 18, pp. 3-114, Akad. Nauk SSSR, Vsesoyuz. Inst. Nauchn. i Tekhn. Informatsii, Moscow, 1981. [English translation: J. Soviet Math. 22 (1983), 1401-1475.] MR633160 (83c:10030)

85. V. Nikulin, Description of automorphism groups of Enriques surfaces (Russian), Dokl. Akad. Nauk SSSR 277 (1984), 1324-1327. MR760514 (86c:14033)

86. V. Nikulin, K3 surfaces with a finite group of automorphisms and a Picard group of rank three (Russian), in "Algebraic geometry and its applications", Trudy Mat. Inst. Steklov. 165 (1984), 119-142. MR752938 (86e:14018)

87. V. Nikulin, Discrete reflection groups in Lobachevsky spaces and algebraic surfaces, Proceedings of the International Congress of Mathematicians, Vols. 1, 2 (Berkeley, Calif., 1986), 654-671, Amer. Math. Soc., Providence, RI, 1987. MR934268 (89d:11032)

88. S. Norton, Constructing the Monster, in "Groups, combinatorics \& geometry (Durham, 1990)", 63-76, London Math. Soc. Lecture Note Ser., 165, Cambridge Univ. Press, Cambridge, 1992. MR1200250 (94c:20034)

89. P. Orlik and L. Solomon, Arrangements defined by unitary reflection groups, Math. Ann. 261 (1982), no. 3, 339-357. MR679795 (84h:14006)

90. P. Orlik and H. Terado, Arrangements of hyperplanes, Springer-Verlag, 1992. MR1217488 (94e:52014)

91. H. Pinkham, Simple elliptic singularities, Del Pezzo surfaces and Cremona transformations, in "Several complex variables (Proc. Sympos. Pure Math., Vol. XXX, Part 1, Williams Coll., Williamstown, Mass., 1975)", pp. 69-71. Amer. Math. Soc., Providence, RI, 1977. MR0441969 (56:358)

92. H. Pinkham, Résolution simultanée de points doubles rationnels in "Séminaire sur les Singularités des Surfaces", ed. by M. Demazure, H. Pinkham and B. Teissier. Lecture Notes in Mathematics, 777. Springer, Berlin, 1980. MR579026 (82d:14021)

93. V. Popov, Discrete complex reflection groups, Communications of the Mathematical Institute, Rijksuniversiteit Utrecht, 15. Rijksuniversiteit Utrecht, Mathematical Institute, Utrecht, 1982, 89 pp. MR645542 (83g:20049)

94. I. Pjatecki-Shapiro and I. Shafarevich, Torelli's theorem for algebraic surfaces of type K3, Izv. Akad. Nauk SSSR Ser. Mat. 35 (1971), 530-572. [English translation: Math. USSR Izv. 5 (1971), 547-587.] MR0284440 (44:1666)

95. M. Prokhorov, Absence of discrete groups of reflections with a noncompact fundamental polyhedron of finite volume in a Lobachevskii space of high dimension (Russian), Izv. Akad. Nauk SSSR Ser. Mat. 50 (1986), no. 2, 413-424. MR842588 (87k:22016)

96. A. Rudakov and I. Shafarevich, Surfaces of type $K 3$ over fields of finite characteristic (Russian), Current problems in mathematics, Vol. 18, pp. 115-207, Akad. Nauk SSSR, Vsesoyuz. Inst. Nauchn. i Tekhn. Informatsii, Moscow, 1981. [English translation: I. Shafarevich, Collected mathematical papers, Springer-Verlag, Berlin, 1989.] MR633161 (83c:14027), MR0977275 (89m:01142)

97. R. Scharlau and C. Walhorn, Integral lattices and hyperbolic reflection groups, Journées Arithmétiques, 1991 (Geneva). Astérisque No. 209 (1992), 279-291. MR1211022 (94j:11057) 
98. P. Schoute, Over het verband tusschen de hoekpunten van een bepaald zesdimsionaal polytoop en de rechten van een kubisch oppervlak, Proc. Konig. Akad. Wis. Amsterdam 19 (1910), 375-383.

99. J-P. Serre, A course in arithmetic, Translated from the French. Graduate Texts in Mathematics, No. 7. Springer-Verlag, New York-Heidelberg, 1973. MR0344216 (49:8956)

100. F. Severi, Complementi alla teoria della base per la totalite delle curve di una superficie algebrica. Palermo Rend. 30 (1910), 265-288.

101. I. Shafarevich, Le théorème de Torelli pour les surfaces algébriques de type K3. Actes du Congrès International des Mathématiciens (Nice, 1970), Tome 1, pp. 413-417. GauthierVillars, Paris, 1971. MR0419459 (54:7480)

102. G. Shephard and J. Todd, Finite unitary reflection groups, Canadian J. Math. 6 (1954). 274-304. MR0059914 (15:600b)

103. O. Shvarzman, Reflectivity of three-dimensional hyperbolic lattices (Russian), Problems in group theory and homological algebra (Russian), 135-141, Matematika, Yaroslavl Gos. Univ., Yaroslavl, 1990. MR1169974 (93d:51031)

104. C. Siegel, Über die analytische Theorie der quadratischen Formen, Ann. of Math. (2) 36 (1935), no. 3, 527-606. MR1503238

105. P. Slodowy, Simple singularities and simple algebraic groups, Lecture Notes in Mathematics, 815. Springer, Berlin, 1980. MR584445 (82g:14037)

106. P. Slodowy, Four lectures on simple groups and singularities, Communications of the Mathematical Institute, Rijksuniversiteit Utrecht, 11. Rijksuniversiteit Utrecht, Mathematical Institute, Utrecht, 1980. MR563725 (82b:14002)

107. P. Slodowy, Simple singularities and complex reflections, in "New developments in singularity theory (Cambridge, 2000)", 329-348, NATO Sci. Ser. II Math. Phys. Chem., 21, Kluwer Acad. Publ., Dordrecht, 2001. MR1849315 (2002h:14061)

108. J. Steenbrink, Mixed Hodge structures associated with isolated singularities, in "Singularities, Part 2 (Arcata, Calif., 1981)", 513-536, Proc. Sympos. Pure Math., v. 40, Amer. Math. Soc., Providence, RI, 1983. MR713277 (85d:32044)

109. T. Terada, Problème de Riemann et fonctions automorphes provenant des fonctions hypergéométriques de plusieurs variables, J. Math. Kyoto Univ. 13 (1973), 557-578. MR0481156 (58:1299)

110. W. Thurston, Shapes of polyhedra and triangulations of the sphere, The Epstein birthday schrift, 511-549 (electronic), Geom. Topol. Monogr., 1, Geom. Topol. Publ., Coventry, 1998. MR1668340 (2000b:57026)

111. G. Tjurina, Resolution of singularities of flat deformations of double rational points, Funkcional. Anal. i Priložen. 4 (1970), no. 1, 77-83. MR0267129 (42:2031)

112. E. Vinberg, Discrete groups generated by reflections in Lobachevskii spaces (Russian), Mat. Sb. (N.S.) 72 (114) (1967), 471-488; correction, ibid. 73 (115) (1967), 303. MR0207853 $(34: 7667)$

113. E. Vinberg, The groups of units of certain quadratic forms (Russian), Mat. Sb. (N.S.) 87 (129) (1972), 18-36. MR0295193 (45:4261)

114. E. Vinberg, Some arithmetical discrete groups in Lobachevsky space, in "Discrete subgroups of Lie groups and applications to moduli", Internat. Colloq., Bombay, 1973, pp. 323-348. Oxford Univ. Press, Bombay, 1975. MR0422505 (54:10492)

115. E. Vinberg, The two most algebraic K3 surfaces, Math. Ann. 265 (1983), no. 1, 1-21. MR719348 (85k:14020)

116. E. Vinberg, Absence of crystallographic groups of reflections in Lobachevski spaces of large dimension (Russian), Trudy Moskov. Mat. Obshch. 47 (1984), 68-102, 246. MR774946 (86i:22020)

117. E. Vinberg, Discrete reflection groups in Lobachevsky spaces, Proceedings of the International Congress of Mathematicians, Vols. 1, 2 (Warsaw, 1983), 593-601, PWN, Warsaw, 1984. MR804716 (87h:22016)

118. E. Vinberg, Hyperbolic groups of reflections (Russian), Uspekhi Mat. Nauk 40 (1985), no. 1 (241), 29-66, 255. [English transl.: Russian Math. Surveys 40 (1985), no. 1, 31-75.] MR783604 (86m:53059)

119. E. Vinberg and I. Kaplinskaja, The groups $O_{18,1}(Z)$ and $O_{19,1}(Z)$ (Russian), Dokl. Akad. Nauk SSSR 238 (1978), no. 6, 1273-1275. MR0476640 (57:16199) 
120. E. Vinberg and O. Shvartsman, Discrete groups of motions of spaces of constant curvature, Geometry, II, 139-248, Encyclopaedia Math. Sci., 29, Springer, Berlin, 1993. MR1254933 (95b:53043)

121. C. T. C. Wall, A note on symmetry of singularities, Bull. London Math. Soc. 12 (1980), 169-175. MR572095 (81f:32009)

Department of Mathematics, University of Michigan, 525 E. University Avenue, Ann Arbor, Michigan 49109

E-mail address: idolga@umich.edu 